\title{
Assessment of injury in reperfused acute myocardial infarction using cardiovascular magnetic resonance imaging
}

Citation for published version (APA):

Bekkers, S. C. A. M. (2011). Assessment of injury in reperfused acute myocardial infarction using cardiovascular magnetic resonance imaging. [Doctoral Thesis, Maastricht University]. Maastricht University. https://doi.org/10.26481/dis.20110601sb

Document status and date:

Published: 01/01/2011

DOI:

10.26481/dis.20110601sb

Document Version:

Publisher's PDF, also known as Version of record

Please check the document version of this publication:

- A submitted manuscript is the version of the article upon submission and before peer-review. There can be important differences between the submitted version and the official published version of record.

People interested in the research are advised to contact the author for the final version of the publication, or visit the DOI to the publisher's website.

- The final author version and the galley proof are versions of the publication after peer review.

- The final published version features the final layout of the paper including the volume, issue and page numbers.

Link to publication

\footnotetext{
General rights rights.

- You may freely distribute the URL identifying the publication in the public portal. please follow below link for the End User Agreement:

www.umlib.nl/taverne-license

Take down policy

If you believe that this document breaches copyright please contact us at:

repository@maastrichtuniversity.nl

providing details and we will investigate your claim.
}

Copyright and moral rights for the publications made accessible in the public portal are retained by the authors and/or other copyright owners and it is a condition of accessing publications that users recognise and abide by the legal requirements associated with these

- Users may download and print one copy of any publication from the public portal for the purpose of private study or research.

- You may not further distribute the material or use it for any profit-making activity or commercial gain

If the publication is distributed under the terms of Article 25fa of the Dutch Copyright Act, indicated by the "Taverne" license above, 
Assessment of injury in reperfused acute myocardial infarction using cardiovascular magnetic resonance imaging 
(C) S.C.A.M. Bekkers, Maastricht 2011

\section{ISBN 978-90-9026094-5}

Cover: A. Kaemingk, Maastricht

Image: Eenentwintigste aftekening door Gerard de Lairesse uit 'Ontleding Des Menschelyken lichaams', gedaan en beschreven door Govard Bidloo

Printing: T.O. Offset-Gijsemberg 


\title{
Assessment of injury in reperfused acute myocardial infarction using cardiovascular magnetic resonance imaging
}

\author{
PROEFSCHRIFT \\ ter verkrijging van de graad van doctor aan de Universiteit Maastricht, \\ op gezag van de Rector Magnificus, Prof. mr. G.P.M.F. Mols, \\ volgens het besluit van het College van Decanen, \\ in het openbaar te verdedigen \\ op woensdag 1 juni 2011 om 10:00 uur
}

door

Sebastiaan Cornelis Antonius Maria Bekkers 


\section{Promotores}

Prof. dr. A.P.M. Gorgels

Prof. dr. J.L. Waltenberger

\section{Beoordelingscommissie}

Prof. dr. J.E. Wildberger (voorzitter)

Prof. dr. H.J.G.M. Crijns

Prof. dr. M.J. Daemen

Prof. dr. F.E. Rademakers, Universitair Ziekenhuis Leuven, België

Prof. dr. A.C. van Rossum, VU Medisch Centrum, Amsterdam

This study was performed in the Maastricht University Medical Center, the Netherlands and supported by a grant from the Profileringsfonds (PF-226). Financial support by 'Stichting Hartsvrienden RESCAR Maastricht' and the Maastricht University Medical Center for the publication of this thesis is gratefully acknowledged.

Additional financial support was kindly provided by Astellas Pharma BV, AstraZeneca BV, Biotronik Nederland BV, Boehringer Ingelheim BV, Boston Scientific Nederland BV, Daiichi-Sankyo Nederland BV, Lamepro BV, Meda Pharma BV, Menarini Farma Nederland, Merck Sharp \& Dohme BV, Novartis Pharma BV, Pfizer BV, Philips Healthcare, Pie Medical Imaging BV, Sanofi Aventis Nederland BV. 
Aan mijn (groot)ouders, wiens DNA ik deel.

Aan Netty, die het hare met mij deelde. Aan Sam en Merel, die dat van ons delen. 

Chapter 1 General introduction 9

Chapter 2 Microvascular obstruction: underlying pathophysiology and $\quad 19$ clinical diagnosis

Chapter 3 Detection and characteristics of microvascular obstruction in reperfused acute myocardial infarction using an optimized protocol for contrast-enhanced cardiovascular magnetic resonance imaging

Chapter 4 Clinical implications of microvascular obstruction and intramyocardial hemorrhage in acute myocardial infarction using cardiovascular magnetic resonance imaging

Chapter 5 ST segment recovery in relation to underlying infarct characteristics and adverse remodeling as assessed by cardiovascular magnetic resonance imaging

Chapter 6 The performance of angiographic, electrocardiographic, and magnetic resonance imaging methods to assess the area at risk in acute myocardial infarction

Chapter 7 General discussion

Summary

Samenvatting

Dankwoord

Curriculum vitae

List of publications 

CHAPTER 1

General introduction 


\section{Acute myocardial infarction}

Although mortality rates have declined the last decades, ischemic heart disease (IHD) is still a leading cause of death, accounting for $12 \%$ of all deaths worldwide and $7.9 \%$ in the Netherlands in 2009. ${ }^{1,2}$ Of these deaths from IHD, 67\% were attributable to acute myocardial infarction (AMI). ${ }^{2}$ In AMI, occlusive coronary artery thrombosis results in myocardial ischemia and necrosis, firstly starting in the subendocardial myocardium and subsequently progressing as a 'wavefront' toward the epicardium. ${ }^{3,4}$ Without restoration of blood flow, the acutely hypoperfused region of the infarct related artery, the area at risk (AAR), will be subject to near transmural necrosis after 6 hours.

Timely reperfusion therapy with either direct percutaneous coronary intervention ( $\mathrm{PCl}$ ) or thrombolytic agents has significantly reduced mortality and AMI complications. ${ }^{5,6}$ Compared with the prereperfusion era, ventricular arrhythmias, cardiogenic shock, cardiac rupture, and fatal ventricular arrhythmias are less common complications nowadays. Despite successful restoration of epicardial coronary artery patency, adequate reperfusion at the microvascular level is not always established. This phenomenon of 'no-reflow' or microvascular obstruction (MVO) has been observed both experimentally as well as clinically. ${ }^{7,8}$ Potential mechanisms of MVO include regional endothelial cell swelling, swollen surrounding myocytes, infiltration and activation of neutrophils and platelets and the deposition of fibrin that occlude the capillaries. ${ }^{9}$ In addition, coronary microembolization of atherosclerotic debris after $\mathrm{PCl}$ may be responsible for a substantial part of clinically observed MVO. ${ }^{10}$ Reperfused AMI also frequently results in intramyocardial hemorrhage (IMH), which is believed to be the result of leakage of erythrocytes from the severely damaged microvasculature. ${ }^{11,12}$ Myocyte necrosis, MVO and IMH are strong-ly related, time-sensitive phenomena and the real mechanisms underlying these features are not yet well understood in humans. Animal studies have shown that both MVO and IMH depend on the duration of coronary occlusion but lag behind myocyte death. ${ }^{13-16}$ Although MVO and IMH increase with duration of reperfusion ${ }^{17,} 18$, there is still lack of clinical evidence that this increase causes additional myocyte death or that its prevention would salvage any additional myocytes. ${ }^{9}$

Prognosis after AMI has been mainly related to end-systolic volume index (LVESVi) and left ventricular ejection fraction (LVEF). ${ }^{19,20}$ In addition to these traditional risk factors, MVO as assessed with myocardial contrast echocardiography (MCE) and coronary angiography, has also been shown to predict adverse ventricular remodeling and clinical outcome. ${ }^{21-23}$ However, the published reports on the prognostic significance of MVO are inconsistent, partly because studies have used different techniques to assess MVO. Some studies have suggested that MVO is independently associated with more frequent cardiovascular complications and adverse outcome ${ }^{24-27}$, whereas others have failed to prove this concept. ${ }^{28-31}$ With 
many previous techniques it was not possible to simultaneously assess MVO, infarct size and infarct transmurality in one investigation. Because these infarct characteristics are mutually related, the association between MVO and outcome might therefore be merely associative and mainly determined by infarct size.

\section{Cardiovascular magnetic resonance}

\section{Assessment of function and infarction}

Cardiovascular magnetic resonance (CMR) has emerged as a useful tool to comprehensively examine multiple infarct characteristics in one investigation. It consists of multiple distinct techniques that each provide separate pieces of information. Cine CMR, using a balanced steady-state free procession sequence, is the current standard to accurately measure volumes and systolic function and with better interstudy reproducibility than echocardiography. ${ }^{32}$ It is therefore a powerful tool for global evaluation of post-infarction left ventricular remodeling.

The technique of contrast-enhanced CMR (CE-CMR) - late gadolinium enhancement or delayed enhancement CMR (LGE- or DE-CMR) - accurately measures infarct size in vivo in both acute and chronic settings. ${ }^{33}$ The technique involves the administration of gadolinium-based contrast media that decrease T1-relaxation time of infarcted tissue. It is believed that this increase in T1-relaxivity is caused by a larger volume of distribution and retention of Gadolinium in edematous (in AMI) and scar tissue (in chronic MI). Using a T1 weighted (T1W) pulse sequence, infarcted myocardium appears bright (hyperenhanced) relative to normal myocardium. Currently, the most widely used method is an ECG-triggered segmented inversion-recovery fast gradient echo pulse sequence with application of a preparatory pulse to null normal myocardium. ${ }^{34}$ DE-CMR is commonly carried out at 10-15 minutes after contrast administration, which produces greater contrast between normal and infarcted tissue as more Gadolinium washes out the normal myocardium and accumulates in infarcted tissue.

Infarct size measurement by DE-CMR has been shown to provide an added value to the traditional predictors of adverse outcome, LVESVi and LVEF. ${ }^{35,36} \mathrm{Be}-$ cause of its high spatial resolution, DE-CMR allows determining the transmural extent of infarction, which has been shown to provide supplemental information to infarct size in predicting improvement of contractile function. ${ }^{37,38}$

\section{Detection of MVO and IMH}

MVO can be detected with CMR using different contrast-enhanced techniques, but a gold standard has not yet been established. Due to differences in wash-in and 
wash-out kinetics as compared to normal and infarcted myocardium, MVO can either be shown as hypoenhanced areas 1-2 minutes after contrast administration using first pass perfusion CMR (FPP-CMR, early MVO) ${ }^{18,}{ }^{39}$ or as hypoenhanced areas within hyperenhanced areas using traditional DE-CMR (late MVO). ${ }^{24,25}$ Microvascular flow after reperfusion is spatially and temporarily complex. ${ }^{40}$ The extent of MVO decreases going from early to late imaging which is caused by slow diffusion of gadolinium into the infarct core. ${ }^{41}$ The dynamic changes in appearance of MVO in relation to infarct characteristics and remodeling are unknown.

Occlusion and subsequent reperfusion of a coronary artery leads to inflammation and myocardial tissue edema in the vascular bed downstream of the occlusion site. This increased mobile water content causes a prolongation of T2-relaxation which can be detected on T2-weighted ( $T 2 \mathrm{~W}$ ) sequences as areas of increased signal intensity. ${ }^{42,43}$ Studies have shown that central areas of hypoenhancement within these hyperintense areas on T2W imaging correspond to IMH. ${ }^{44-46}$

Conflicting results have been published on the clinical significance of MVO and IMH beyond infarct size. ${ }^{25,27,29-31,47-50}$ This may largely be explained by their mutual correlation with infarct size. ${ }^{11,13}$

\section{Early risk stratification}

Early identification of patients with a good or an unfavorable prognosis remains crucial to decide if patients may need adjunctive infarct size limiting therapies. ${ }^{51}$ Incomplete resolution of ST segment elevation (STR) early after reperfusion has prognostic significance and is widely accepted to reflect compromised myocardial perfusion, even after successful epicardial flow establishment. ${ }^{52-55}$ However, only a limited number of studies has directly related incomplete STR to microvascular dysfunction. ${ }^{56,57}$ The relation of STR with underlying infarct characteristics and adverse remodeling as assessed by CMR are not well known.

\section{The area at risk}

Accurate measurement of the AAR and infarct size is important, because this would allow the assessment of myocardial salvage and the efficacy of reperfusion therapies. For measuring the AAR, electrocardiographic and angiographic methods have been used. ${ }^{58,59}$ Several studies also suggest that the hyperintense area on T2W images delineates the AAR. ${ }^{60,61}$ A novel CMR method for measuring the AAR is the determination of infarct endocardial surface area (ESA) by DE-CMR. ${ }^{62}$ Although promising, validation of these techniques against a proper reference standard is limited. 


\section{Objectives of this thesis}

1. To assess characteristics of injury in reperfused AMI using CMR and investigate their clinical significance in relation to adverse remodeling.

2. To relate early electrocardiographic measures of reperfusion (ST segment resolution) with underlying infarct characteristics and remodeling as assessed with CMR.

3. To evaluate the performance of different AAR measurement methods using established physiological concepts as a reference standard.

\section{Outline of the thesis}

In chapter 2, the underlying pathophysiology of myocardial ischemia with an emphasis on MVO and current techniques to detect MVO are reviewed.

In chapter 3, the prevalence and dynamic changes of MVO over time are evaluated using an optimized technique for early and traditional late contrast-enhanced CMR.

In chapter 4, the clinical implications of $\mathrm{MVO}$ and IMH in AMI regarding adverse remodeling are investigated.

In chapter 5, the relation of early STR to underlying infarct characteristics and adverse remodeling as assessed with CMR are investigated.

In chapter 6, established physiological concepts were used to evaluate the performance of angiographic, electrocardiographic and CMR methods to assess the AAR in AMI.

Chapter 7 discusses the results of this thesis in the light of existing evidence or controversy and directions for future research will be given. 


\section{References}

1. World Health Organization. Fact Sheet No 310 'The top 10 causes of death'. Updated October 2008; Available from: http://www.who.int/mediacentre/factsheets/fs310/en/index.htm.

2. Vaartjes I, van Dis I, Visseren F, et al. Hart- en Vaatziekten in Nederland, 2010. Cijfers over leefstijlen risicofactoren, ziekten en sterfte. Den Haag: Nederlandse Hartstichting. 2010:7-28.

3. Davies MJ, Woolf N, Robertson WB. Pathology of acute myocardial infarction with particular reference to occlusive coronary thrombi. Br Heart J. Jul 1976;38(7):659-664.

4. Reimer KA, Lowe JE, Rasmussen MM, et al. The wavefront phenomenon of ischemic cell death. 1. Myocardial infarct size vs duration of coronary occlusion in dogs. Circulation. Nov 1977;56(5):786794.

5. De Luca G, Suryapranata H, Ottervanger JP, et al. Time delay to treatment and mortality in primary angioplasty for acute myocardial infarction: every minute of delay counts. Circulation. Mar 16 2004;109(10):1223-1225.

6. Keeley EC, Boura JA, Grines CL. Primary angioplasty versus intravenous thrombolytic therapy for acute myocardial infarction: a quantitative review of 23 randomised trials. Lancet. Jan 4 2003;361(9351):13-20.

7. Ito H, Tomooka T, Sakai N, et al. Lack of myocardial perfusion immediately after successful thrombolysis. A predictor of poor recovery of left ventricular function in anterior myocardial infarction. Circulation. May 1992;85(5):1699-1705.

8. Kloner RA, Ganote CE, Whalen DA, Jr., et al. Effect of a transient period of ischemia on myocardial cells. II. Fine structure during the first few minutes of reflow. Am J Pathol. Mar 1974;74(3):399-422.

9. Reffelmann T, Kloner RA. The no-reflow phenomenon: A basic mechanism of myocardial ischemia and reperfusion. Basic Res Cardiol. Sep 2006;101(5):359-372.

10. Henriques JP, Zijlstra F, Ottervanger JP, et al. Incidence and clinical significance of distal embolization during primary angioplasty for acute myocardial infarction. Eur Heart J. Jul 2002;23(14):11121117.

11. Garcia-Dorado D, Theroux P, Solares J, et al. Determinants of hemorrhagic infarcts. Histologic observations from experiments involving coronary occlusion, coronary reperfusion, and reocclusion. Am J Pathol. Aug 1990;137(2):301-311.

12. Higginson LA, White F, Heggtveit HA, et al. Determinants of myocardial hemorrhage after coronary reperfusion in the anesthetized dog. Circulation. Jan 1982;65(1):62-69.

13. Fishbein $M C, J Y R$, Lando $U$, et al. The relationship of vascular injury and myocardial hemorrhage to necrosis after reperfusion. Circulation. Dec 1980;62(6):1274-1279.

14. Higginson LA, Beanlands DS, Nair RC, et al. The time course and characterization of myocardial hemorrhage after coronary reperfusion in the anesthetized dog. Circulation. May 1983;67(5):10241031.

15. Kloner RA, Ganote CE, Jennings RB. The "no-reflow" phenomenon after temporary coronary occlusion in the dog. J Clin Invest. Dec 1974;54(6):1496-1508.

16. Reffelmann T, Hale SL, Li G, et al. Relationship between no reflow and infarct size as influenced by the duration of ischemia and reperfusion. Am J Physiol Heart Circ Physiol. Feb 2002;282(2):H766772.

17. Ambrosio G, Weisman HF, Mannisi JA, et al. Progressive impairment of regional myocardial perfusion after initial restoration of postischemic blood flow. Circulation. Dec 1989;80(6):1846-1861.

18. Rochitte CE, Lima JA, Bluemke DA, et al. Magnitude and time course of microvascular obstruction and tissue injury after acute myocardial infarction. Circulation. Sep 8 1998;98(10):1006-1014.

19. White HD, Norris RM, Brown MA, et al. Left ventricular end-systolic volume as the major determinant of survival after recovery from myocardial infarction. Circulation. Jul 1987;76(1):44-51.

20. Burns RJ, Gibbons RJ, Yi Q, et al. The relationships of left ventricular ejection fraction, end-systolic volume index and infarct size to six-month mortality after hospital discharge following myocardial infarction treated by thrombolysis. J Am Coll Cardiol. Jan 2 2002;39(1):30-36. 
21. Bolognese L, Carrabba N, Parodi G, et al. Impact of microvascular dysfunction on left ventricular remodeling and long-term clinical outcome after primary coronary angioplasty for acute myocardial infarction. Circulation. Mar 9 2004;109(9):1121-1126.

22. Ito H, Maruyama A, Iwakura K, et al. Clinical implications of the 'no reflow' phenomenon. A predictor of complications and left ventricular remodeling in reperfused anterior wall myocardial infarction. Circulation. Jan 15 1996;93(2):223-228.

23. Morishima I, Sone T, Okumura K, et al. Angiographic no-reflow phenomenon as a predictor of adverse long-term outcome in patients treated with percutaneous transluminal coronary angioplasty for first acute myocardial infarction. J Am Coll Cardiol. Oct 2000;36(4):1202-1209.

24. Hombach V, Grebe O, Merkle N, et al. Sequelae of acute myocardial infarction regarding cardiac structure and function and their prognostic significance as assessed by magnetic resonance imaging. Eur Heart J. Mar 2005;26(6):549-557.

25. Nijveldt R, Beek AM, Hirsch A, et al. Functional recovery after acute myocardial infarction: comparison between angiography, electrocardiography, and cardiovascular magnetic resonance measures of microvascular injury. J Am Coll Cardiol. Jul 15 2008;52(3):181-189.

26. Orn S, Manhenke C, Greve OJ, et al. Microvascular obstruction is a major determinant of infarct healing and subsequent left ventricular remodelling following primary percutaneous coronary intervention. Eur Heart J. Jun 62009.

27. Wu KC, Zerhouni EA, Judd RM, et al. Prognostic significance of microvascular obstruction by magnetic resonance imaging in patients with acute myocardial infarction. Circulation. Mar 3 1998;97(8):765-772.

28. Baks T, van Geuns RJ, Biagini E, et al. Recovery of left ventricular function after primary angioplasty for acute myocardial infarction. Eur Heart J. Jun 2005;26(11):1070-1077.

29. Larose E, Rodes-Cabau J, Pibarot P, et al. Predicting late myocardial recovery and outcomes in the early hours of ST-segment elevation myocardial infarction traditional measures compared with microvascular obstruction, salvaged myocardium, and necrosis characteristics by cardiovascular magnetic resonance. J Am Coll Cardiol. Jun 1 2010;55(22):2459-2469.

30. Shapiro MD, Nieman K, Nasir K, et al. Utility of cardiovascular magnetic resonance to predict left ventricular recovery after primary percutaneous coronary intervention for patients presenting with acute ST-segment elevation myocardial infarction. Am J Cardiol. Jul 15 2007;100(2):211-216.

31. Tarantini G, Razzolini R, Cacciavillani L, et al. Influence of transmurality, infarct size, and severe microvascular obstruction on left ventricular remodeling and function after primary coronary angioplasty. Am J Cardiol. Oct 15 2006;98(8):1033-1040.

32. Grothues F, Moon JC, Bellenger NG, et al. Interstudy reproducibility of right ventricular volumes, function, and mass with cardiovascular magnetic resonance. Am Heart J. Feb 2004;147(2):218-223.

33. Thiele H, Kappl MJ, Conradi S, et al. Reproducibility of chronic and acute infarct size measurement by delayed enhancement-magnetic resonance imaging. J Am Coll Cardiol. Apr 18 2006;47(8):16411645.

34. Kim RJ, Shah DJ, Judd RM. How we perform delayed enhancement imaging. J Cardiovasc Magn Reson. Jul 2003;5(3):505-514.

35. Cheong BY, Muthupillai R, Wilson JM, et al. Prognostic significance of delayed-enhancement magnetic resonance imaging: survival of 857 patients with and without left ventricular dysfunction. Circulation. Nov 24 2009;120(21):2069-2076.

36. Wu E, Ortiz JT, Tejedor P, et al. Infarct size by contrast enhanced cardiac magnetic resonance is a stronger predictor of outcomes than left ventricular ejection fraction or end-systolic volume index: prospective cohort study. Heart. Jun 2008;94(6):730-736.

37. Gerber BL, Garot J, Bluemke DA, et al. Accuracy of contrast-enhanced magnetic resonance imaging in predicting improvement of regional myocardial function in patients after acute myocardial infarction. Circulation. Aug 27 2002;106(9):1083-1089.

38. Kim RJ, Wu E, Rafael A, et al. The use of contrast-enhanced magnetic resonance imaging to identify reversible myocardial dysfunction. N Engl J Med. Nov 16 2000;343(20):1445-1453. 
39. Judd RM, Lugo-Olivieri $\mathrm{CH}$, Arai M, et al. Physiological basis of myocardial contrast enhancement in fast magnetic resonance images of 2-day-old reperfused canine infarcts. Circulation. Oct 1 1995;92(7):1902-1910.

40. Villanueva FS. Myocardial contrast echocardiography in acute myocardial infarction. Am J Cardiol. Nov 18 2002;90(10A):38J-47J.

41. Nijveldt R, Hofman MB, Hirsch A, et al. Assessment of microvascular obstruction and prediction of short-term remodeling after acute myocardial infarction: cardiac MR imaging study. Radiology. Feb 2009;250(2):363-370.

42. Garcia-Dorado D, Oliveras J, Gili J, et al. Analysis of myocardial oedema by magnetic resonance imaging early after coronary artery occlusion with or without reperfusion. Cardiovasc Res. Aug 1993;27(8):1462-1469.

43. Higgins $C B$, Herfkens R, Lipton MJ, et al. Nuclear magnetic resonance imaging of acute myocardial infarction in dogs: alterations in magnetic relaxation times. Am J Cardiol. Jul 1983;52(1):184-188.

44. Basso C, Corbetti F, Silva C, et al. Morphologic validation of reperfused hemorrhagic myocardial infarction by cardiovascular magnetic resonance. Am J Cardiol. Oct 15 2007;100(8):1322-1327.

45. Lotan CS, Bouchard A, Cranney GB, et al. Assessment of postreperfusion myocardial hemorrhage using proton NMR imaging at 1.5 T. Circulation. Sep 1992;86(3):1018-1025.

46. O'Regan DP, Ahmed R, Karunanithy N, et al. Reperfusion hemorrhage following acute myocardial infarction: assessment with T2* mapping and effect on measuring the area at risk. Radiology. Mar 2009;250(3):916-922.

47. Beek AM, Nijveldt R, van Rossum AC. Intramyocardial hemorrhage and microvascular obstruction after primary percutaneous coronary intervention. Int J Cardiovasc Imaging. Sep 152009.

48. Bondarenko O, Beek AM, Hofman MB, et al. Standardizing the definition of hyperenhancement in the quantitative assessment of infarct size and myocardial viability using delayed contrast-enhanced CMR. J Cardiovasc Magn Reson. 2005;7(2):481-485.

49. Ganame J, Messalli G, Dymarkowski S, et al. Impact of myocardial haemorrhage on left ventricular function and remodelling in patients with reperfused acute myocardial infarction. Eur Heart J. Jun 2009;30(12):1440-1449.

50. Mather AN, Fairbairn TA, Ball SG, et al. Reperfusion haemorrhage as determined by cardiovascular MRI is a predictor of adverse left ventricular remodelling and markers of late arrhythmic risk. Heart. Nov 42010.

51. Yellon DM, Hausenloy DJ. Myocardial reperfusion injury. N Engl J Med. Sep 13 2007;357(11):11211135.

52. Buller CE, Fu Y, Mahaffey KW, et al. ST-segment recovery and outcome after primary percutaneous coronary intervention for ST-elevation myocardial infarction: insights from the Assessment of Pexelizumab in Acute Myocardial Infarction (APEX-AMI) trial. Circulation. Sep 23 2008;118(13):13351346.

53. de Lemos JA, Braunwald E. ST segment resolution as a tool for assessing the efficacy of reperfusion therapy. J Am Coll Cardiol. Nov 1 2001;38(5):1283-1294.

54. Schroder R. Prognostic impact of early ST-segment resolution in acute ST-elevation myocardial infarction. Circulation. Nov 23 2004;110(21):e506-510.

55. van 't Hof AW, Liem A, de Boer MJ, et al. Clinical value of 12-lead electrocardiogram after successful reperfusion therapy for acute myocardial infarction. Zwolle Myocardial infarction Study Group. Lancet. Aug 30 1997;350(9078):615-619.

56. Feldman LJ, Coste P, Furber A, et al. Incomplete resolution of ST-segment elevation is a marker of transient microcirculatory dysfunction after stenting for acute myocardial infarction. Circulation. Jun 3 2003;107(21):2684-2689.

57. Santoro GM, Valenti R, Buonamici P, et al. Relation between ST-segment changes and myocardial perfusion evaluated by myocardial contrast echocardiography in patients with acute myocardial infarction treated with direct angioplasty. Am J Cardiol. Oct 15 1998;82(8):932-937. 
58. Aldrich HR, Wagner NB, Boswick J, et al. Use of initial ST-segment deviation for prediction of final electrocardiographic size of acute myocardial infarcts. Am J Cardiol. Apr 1 1988;61(10):749-753.

59. Graham MM, Faris PD, Ghali WA, et al. Validation of three myocardial jeopardy scores in a population-based cardiac catheterization cohort. Am Heart J. Aug 2001;142(2):254-261.

60. Aletras AH, Tilak GS, Natanzon A, et al. Retrospective determination of the area at risk for reperfused acute myocardial infarction with T2-weighted cardiac magnetic resonance imaging: histopathological and displacement encoding with stimulated echoes (DENSE) functional validations. Circulation. Apr 18 2006;113(15):1865-1870.

61. Friedrich MG, Abdel-Aty $\mathrm{H}$, Taylor A, et al. The salvaged area at risk in reperfused acute myocardial infarction as visualized by cardiovascular magnetic resonance. J Am Coll Cardiol. Apr 22 2008;51(16):1581-1587.

62. Ortiz-Perez JT, Meyers SN, Lee DC, et al. Angiographic estimates of myocardium at risk during acute myocardial infarction: validation study using cardiac magnetic resonance imaging. Eur Heart J. Jul 2007;28(14):1750-1758. 


\section{Microvascular obstruction: underlying pathophysiology and clinical diagnosis}

Bekkers SCAM, Yazdani SK, Virmani R, Waltenberger JL Journal of the American College of Cardiology 2010; 55 (16): 1649-60 


\begin{abstract}
Successful restoration of epicardial coronary artery patency after prolonged occlusion might result in microvascular obstruction (MVO) and is observed both experimentally as well as clinically. In reperfused myocardium, myocytes appear edematous and swollen from osmotic overload. Endothelial cell changes usually accompany the alterations seen in myocytes but lag behind myocardial cell injury. Endothelial cells become voluminous, with large intraluminal endothelial protrusions into the vascular lumen, and together with swollen surrounding myocytes occlude capillaries. The infiltration and activation of neutrophils and platelets and the deposition of fibrin also play an important role in reperfusion-induced microvascular damage and obstruction. In addition to these ischemia-reperfusion-related events, coronary microembolization of atherosclerotic debris after percutaneous coronary intervention is responsible for a substantial part of clinically observed MVO. Microvascular flow after reperfusion is spatially and temporally complex. Regions of hyperemia, impaired vasodilatory flow reserve and very low flow coexist and these perfusion patterns vary over time as a result of reperfusion injury. The MVO first appears centrally in the infarct core extending toward the epicardium over time. Accurate detection of MVO is crucial, because it is independently associated with adverse ventricular remodeling and patient prognosis. Several techniques (coronary angiography, myocardial contrast echocardiography, cardiovascular magnetic resonance imaging, electrocardiography) measuring slightly different biological and functional parameters are used clinically and experimentally. Currently there is no consensus as to how and when MVO should be evaluated after acute myocardial infarction.
\end{abstract}


Timely reperfusion in patients with acute myocardial infarction (AMI) salvages myocardium and reduces mortality. ${ }^{1}$ However, successful restoration of epicardial coronary artery patency after prolonged occlusion does not always lead to adequate reperfusion at the microvascular level. This phenomenon of no-reflow or microvascular obstruction (MVO) is observed both experimentally as well as clinically. ${ }^{2,3}$ Accurate detection and quantification of MVO is important, because previous studies have shown that MVO is independently associated with adverse ventricular remodeling and patient prognosis. 2, 4-7 The "open epicardial artery hypothesis" should thus be shifted downstream to an "open micro-vessel hypothesis" for reflecting optimal reperfusion. ${ }^{8}$

Although both the clinical as well as the experimental setting of ischemiareperfusion share important underlying pathophysiologic aspects, the experimental models of coronary occlusion and reperfusion are lacking certain aspects frequently present in the clinical situation. For instance, distal coronary microembolization of atherosclerotic debris or thrombotic material might be responsible for a substantial part of clinically observed MVO, which is not simulated in standard animal models of ischemia and reperfusion. ${ }^{6}$

Imaging techniques play an important role in the development and evaluation of future cardioprotective interventions. ${ }^{9}$ However, a "gold standard" for the detection of MVO has not been established, and several techniques measuring slightly different biological parameters are being used clinically and experimentally. The sensitivity and specificity of all these techniques is as yet undetermined. Following a clinical case presentation, we review the underlying pathophysiology of myocardial ischemia with an emphasis on MVO and review current techniques to detect MVO.

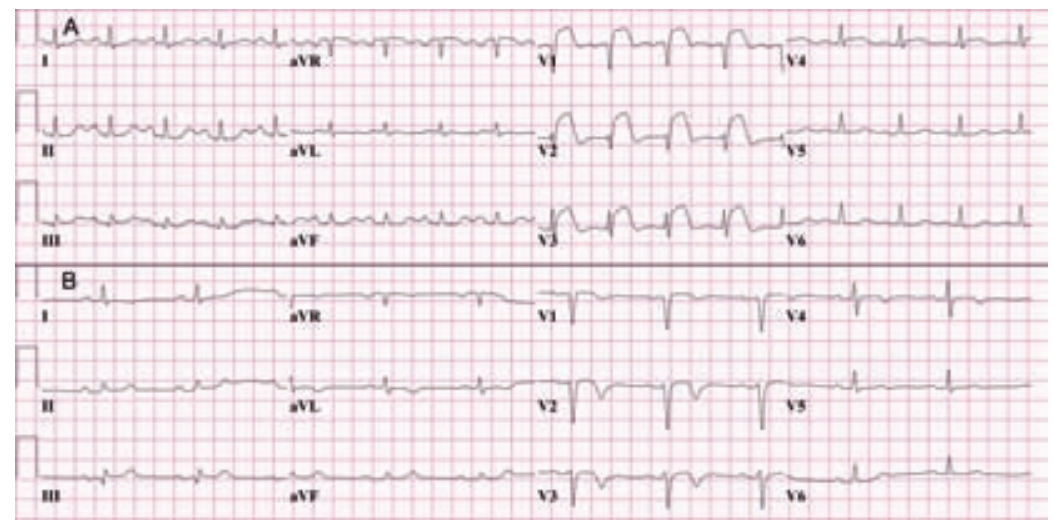

Figure 1. Electrocardiogram on admission and after intervention. (A) The ST-segment elevation in leads $V_{1}$ to $V_{4}$ indicating acute transmural ischemia of the anterior wall. (B) Despite $>70 \%$ resolution of initial ST-segment elevation, persistent ST-segment elevation in leads $V_{2}$ to $V_{3}$ (1 to $2 \mathrm{~mm}$ ) is still present $40 \mathrm{~min}$ after percutaneous coronary intervention. 


\section{Case presentation}

A 49-year-old woman with a history of smoking presented to the emergency department after 3 hours of crushing retrosternal chest pain. The electrocardiogram (ECG) showed ST-segment elevation in leads $V_{1}$ to $V_{4}$, indicating acute transmural anteroseptal ischemia (Figure 1A). She was treated with aspirin $300 \mathrm{mg}$, clopidogrel $600 \mathrm{mg}$, weight-adjusted low-molecular weight heparin, nitroglycerine, a betablocker, and a glycoprotein IIb/IIla inhibitor.

Coronary angiography showed an occlusive thrombus in the mid left anterior descending artery at the bifurcation of the second diagonal branch (Figures $2 \mathrm{~A}$ and 2B). Subsequently and approximately 4 hours after symptom onset, percutaneous coronary intervention $(\mathrm{PCl})$ with thrombosuction and stenting was performed (Figure $2 \mathrm{C}$ ). Despite an angiographically optimal result without residual stenosis, antegrade epicardial flow was only partially restored to Thrombolysis In Myocardial Infarction (TIMI) flow grade 2, even after intracoronary adenosine injection (Figure 2D).

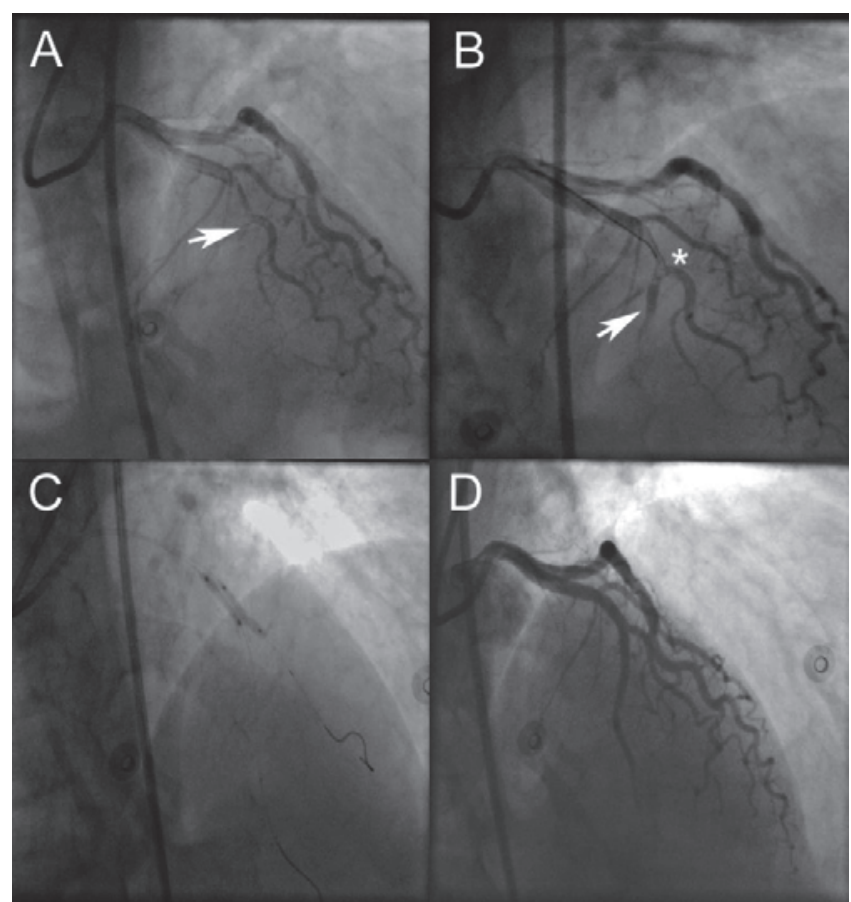

Figure 2. Acute coronary angiogram. Proximal occlusion of the left anterior descending artery (LAD) (A) (arrow). After introduction of the first guide wire, intraluminal thrombus (haziness) at the bifurcation of LAD (arrow) and second diagonal branch $\left({ }^{*}\right)$ can be seen (B). After thrombosuction and stenting followed by kissing balloon inflation (C), flow was only partially restored to Thrombolysis In Myocardial Infarction flow grade 2 , despite an angiographically optimal result (D). 


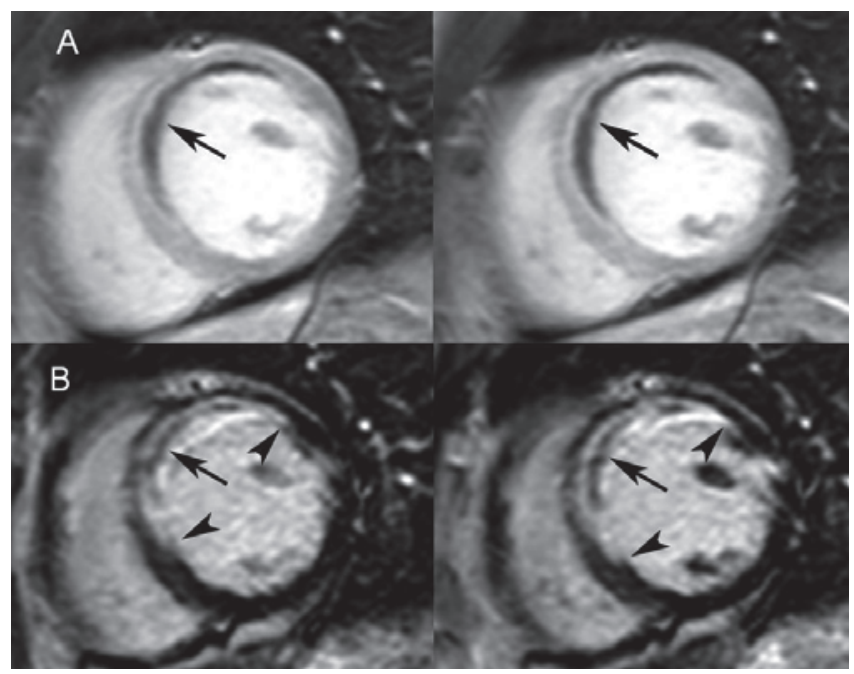

Figure 3. Cardiovascular MRI. A set of 2 corresponding short-axis images acquired with a single breathhold 3-dimensional inversion recovery gradient-echo pulse sequence. (A) Early (2 min after contrast injection) contrast-enhanced cardiovascular magnetic resonance imaging (MRI) showing a central hypoenhanced area corresponding to microvascular obstruction (MVO) in the anteroseptal region (arrows, early MVO). (B) Late gadolinium-enhanced cardiovascular MRI (10 min after contrast injection) showing hyperenhancement indicating an anteroseptal myocardial infarction (arrowheads) with a central zone of MVO (arrows, late MVO). Note that the extent of late MVO is smaller than early MVO, due to contrast penetration over time (see Cardiovascular MRI section).

In the Intensive Cardiac Care Unit, her chest pain completely subsided. Despite significant ST-segment resolution (STR $\geq 70 \%$ ), persistent ST-segment elevation in leads $\mathrm{V}_{1}$ to $\mathrm{V}_{3}$ was still present $40 \mathrm{~min}$ after $\mathrm{PCl}$ (Figure 1B). The peak creatine kinase level of $900 \mathrm{U} / \mathrm{I}$ was reached $12 \mathrm{~h}$ after admission.

Cardiovascular magnetic resonance imaging (MRI) on day 4 demonstrated antero-septal akinesia, a left ventricular end-diastolic volume of $149 \mathrm{ml}$, LV stroke volume of $67 \mathrm{ml}$, and ejection fraction (EF) of 45\%. Early contrast-enhanced cardiovascular MRI performed 2 min after contrast injection showed a central hypointense area in the anteroseptal wall corresponding to MVO (Figure 3A, arrows). Delayed enhancement cardiovascular MRI (DE-CMR) 10 min after contrast injection showed an anteroseptal myocardial infarction (Figure 3B, arrowheads) with a central zone of MVO (Figure 3B, arrows).

Her hospital course was uneventful, and she was discharged on day 6 with aspirin, clopidogrel, statin, angiotensin-converting enzyme inhibitor and beta-blocker. After 3 months she was in New York Heart Association (NYHA) functional class III, and a repeat cardiovascular MRI showed an increased LV end-diastolic volume and decreased EF (41\%). 


\section{Mechanisms of ischemic myocardial Injury}

The anatomic and biochemical events of ischemic cell injury and death have been well-described. 9, 10 Under normal aerobic conditions cardiac energy is acquired from fatty acids, which supply $60 \%$ to $90 \%$ of the energy for adenosine triphosphate (ATP) synthesis. The remaining energy comes from oxidation of pyruvate formed from glycolysis and lactate oxidation. Sudden occlusion of a coronary artery shifts aerobic or mitochondrial metabolism to anaerobic glycolysis within seconds. Reduced aerobic ATP formation stimulates glycolysis and increases myocardial glucose uptake and glycogen breakdown. A decrease in ATP inhibits $\mathrm{Na}^{+} / \mathrm{K}^{+}$-ATPase, increasing intracellular $\mathrm{Na}^{+}$and $\mathrm{Cl}^{-}$, leading to cell swelling. Derangements in transport systems in the sarcolemma and sarcoplasmic reticulum increase cytosolic $\mathrm{Ca}^{2+}$, inducing activation of proteases and alterations in contractile proteins. Pyruvate is not readily oxidized in the mitochondria, leading to production of lactate, fall in intracellular $\mathrm{pH}$, reduction in contractile function, and greater ATP requirement to maintain $\mathrm{Ca}^{2+}$ homeostasis. ${ }^{11}$

\section{Infarct region and Reperfusion}

Infarct size is directly related to the duration of coronary artery occlusion. In animal models, coronary artery occlusion of 15 to $20 \mathrm{~min}$ followed by reperfusion completely prevents myocardial cell necrosis, whereas $40 \mathrm{~min}$ of occlusion results in focal or diffuse subendocardial necrosis involving $38 \pm 4 \%$ of the coronary bed at risk. ${ }^{12}$ At 3 hours of occlusion, myocardial necrosis involves $57 \pm 7 \%$ of the coronary bed, and an additional 3 hours results in an increase of infarct size to $71 \pm 7 \%$, similar to permanent coronary occlusion rates $(79 \pm 6 \%)$. To achieve benefit from reperfusion, as learned in animal models, it is reasonable to administer all therapeutic interventions within the 3-hour "window."

In ischemic but reversibly injured myocardium, myocytes are edematous and swollen from osmotic overload. The cell size is increased with decreased glycogen content. ${ }^{3}$ The myocyte fibrils are relaxed and thinned; I-bands are prominent secondary to noncontracting ischemic myocytes. ${ }^{13}$ The nuclei show mild condensation of chromatin at the nucleoplasm. The cell membrane (sarcolemma) is intact with no breaks. The mitochondria are swollen with loss of normal dense mitochondrial granules or granular flocculent densities. Irreversible injured myocytes contain shrunken nuclei with marked chromatin margination. The 2 hallmarks of irreversible injury are cell membrane breaks and mitochondrial presence of small osmophilic densities. $^{3}$

Endothelial cell (EC) changes of ischemia usually accompany the alterations seen in the myocytes but lag behind myocardial cell injury. ${ }^{14}$ In a canine model, EC 
abnormalities are not seen until $60 \mathrm{~min}$. These abnormalities include focal EC swelling with loss of pinocytotic vesicles, which are observed in $20 \%$ of EC's. ${ }^{14}$ As ischemia advances, the number of affected ECs increases (40\% at 90 to $180 \mathrm{~min}$.). Moreover, as ischemia prolongs, greater endothelial changes result, including clumping and margination of nuclear chromatin, marked swelling of the cytoplasm, formation of intraluminal blebs, and even disruption of EC borders, hemorrhage, and fibrin deposition (Figure 4). ${ }^{14-16}$ The microvasculature can also be obstructed by extravascular compression from myocyte edema. Overall, it seems that microvascular damage lags behind myocardial cell injury, ultrastructurally, and is probably not the primary cause of myocyte injury until reperfusion occurs. ${ }^{14,17}$

\section{The "no-reflow" phenomenon or microvascular obstruction}

A balance exists between the benefits of reperfusion to reduce infarct size and reperfusion injury, which depends on the duration of occlusion. In general, if ischemic myocardium is reperfused early, the degree of myocardial salvage greatly exceeds damage from free radicals and calcium-loading caused by reperfusion. These positive functional consequences of reperfusion are most beneficial within the initial 12 hours after occlusion in humans.

Reperfusion injury is defined as reperfusion-related expansion or worsening of ischemic cardiac injury leading to decreased contractility, an increased arrhythmogenic threshold, conversion of reversible to irreversible myocyte injury, and microvascular dysfunction. ${ }^{3}$ At the time of myocardial reperfusion, there is an abrupt increase in intracellular $\mathrm{Ca}^{2+}$, leading to a disturbance in the normal mechanisms that regulate $\mathrm{Ca}^{2+}$ in the cardiomyocyte, known as the calcium paradox. ${ }^{18}$ This intracellular $\mathrm{Ca}^{2+}$ overload induces death by causing hypercontracture of myocytes and mitochondrial permeability transition pore opening. ${ }^{9,18}$

Microvascular obstruction in the heart was first described by Kloner et al. ${ }^{3}$ and consists of explosive EC swelling on reperfusion of an ischemic bed. ${ }^{19}$ Endothelial protrusion by cell swelling together with neutrophils, red blood cells, and platelets cause capillary obstruction (Figure 4). ${ }^{3}$ Microvessels ultimately rupture, with fibrin and platelet deposition and red and white blood cell extravasation. Furthermore, surrounding myocardial cells swell, potentially compressing capillaries. 


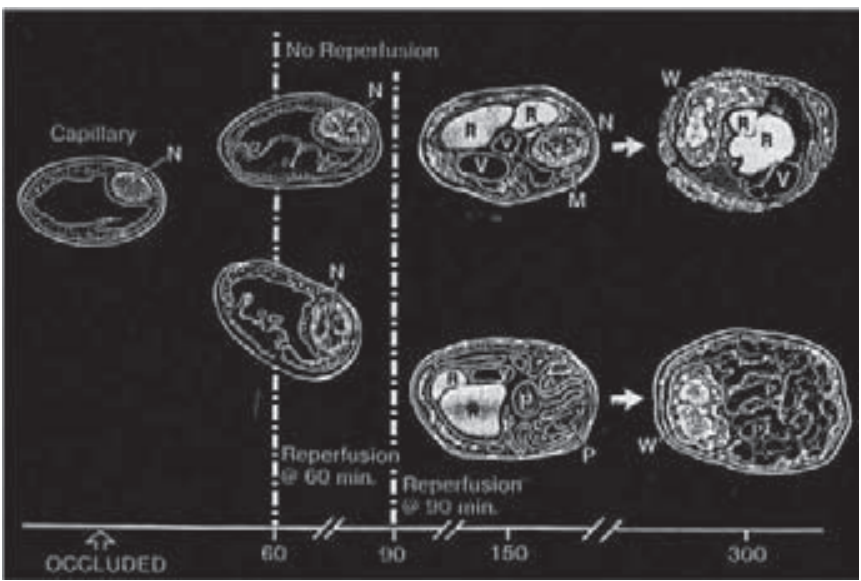

Figure 4. Sequential changes in the microvasculature after varying periods of ischemia. At $60 \mathrm{~min}$. of ischemia, endothelial cells are swollen with decrease in pinocytotic vesicles. Capillary damage (endothelial change) continues to occur with longer duration of ischemia as large intraluminal protrusions $(P)$, absent or decreased pinocytotic vesicles, membrane-bound vesicles (V), and capillary obstruction with red (R) and white (W) blood cell plugging are observed. With permanent coronary occlusion, intravascular protrusions are less pronounced; however, membrane-bound vesicles and red and white blood cell plugging with fibrin deposition are seen only after longer duration of ischemia. $\mathrm{N}=$ nucleus; $\mathrm{M}=$ mitochondrium. Reproduced with permission from Virmani et al. ${ }^{16}$

These changes in the capillary bed can result in poor perfusion of the surrounding potentially viable myocytes with resultant cell death. ${ }^{10,20}$ The failure to reperfuse viable, severely ischemic myocytes (reversible injury) with resultant myocyte cell death (irreversible injury) at the time of reperfusion (the no-reflow phenomenon) has been described after $90 \mathrm{~min}$. in a canine model. ${ }^{3}$ Reperfusion, therefore, as described by Braunwald and Kloner ${ }^{21}$ is a "double-edged" sword, because it can result in the death of potentially salvageable myocardium.

Neutrophils have also been implicated in causing reperfusion injury in the myocardium and other organs. Neutrophils are activated early during myocardial ischemia and precede the appearance of histological tissue injury. ${ }^{17}$ Reperfusion markedly enhances the infiltration of neutrophils into the ischemic region. ${ }^{22}$ The essential initiating step involves interaction of neutrophils with vascular ECs (adhesion). This is followed by activation, diapedesis, and extravascular migration into surrounding myocytes. Production of additional chemoattractants by activated neutrophils amplifies the initial inflammatory response. Neutrophil activation causes a greatly enhanced oxygen uptake by the cell, resulting in the production of large quantities of reactive oxygen species that might lead to disruption of EC and inactivate antiproteases present in the plasma. ${ }^{23}$ 
In addition to these ischemia-reperfusion-related events, distal coronary microembolization of atherosclerotic debris or thrombotic material is responsible for a substantial part of clinically observed MVO ${ }^{6,24}$ (Figure 5). Microemboli are histopathologically associated with MVO, myocyte necrosis and edema, and EC sloughing within the intramyocardial capillaries. ${ }^{25,26}$ The rate of coronary microembolization is highest in documented epicardial coronary thrombosis, reaching $30 \%$ to $54 \%{ }^{27,} 28$ and even higher (79\%) in AMI patients. ${ }^{29}$

Few data compare acute plaque rupture versus acute plaque erosion; however, the authors have noted a higher rate of thrombotic microembolization with plaque erosion. In hearts with acute coronary thrombi, evidence of distal embolization was more frequent in erosions than ruptures. These thrombi were associated with focal myocardial necrosis. Other potential sources of distal embolization include primary $\mathrm{PCl}, \mathrm{PCl}$ of diseased (atherosclerotic) saphenous vein grafts, and thrombolysis. The reported incidence of distal embolization due to soft friable atherosclerotic plaque and adherent thrombus, after $\mathrm{PCl}$ of bypass grafts ranges from $2 \%$ to $42 \%$. ${ }^{30-32} \mathrm{An}$ giographic evidence of distal embolization in patients treated with primary $\mathrm{PCl}$ has been shown to occur in approximately $15 \%$ to $19 \%$ (Figure 6). ${ }^{33,34}$

Microvascular flow within the reperfused bed is both spatially and temporarily complex. Apparently, blood flow in the area of structural MVO is not completely absent but rather very low, ranging between 0.13 and $0.37 \mathrm{ml} / \mathrm{g} / \mathrm{min}$, which is $<50 \%$ of baseline blood flow. ${ }^{35,36}$ Microvascular obstruction first appears centrally in the infarct core extending toward the epicardium over time. Different myocardial regions can be distinguished (Figure 7). ${ }^{37}$ In the noninfarcted region, microvasculature is intact and blood flow is normal. In the noninfarcted (stunned) area-at-risk, microvasculature is structurally and functionally still intact. The infarcted region has outer zones of intact microvasculature with co-existing areas of hyperemia, low reflow, and impaired flow reserve, depending on the degree of capillary damage.

Myocardial blood flow in certain areas of MVO is hyperemic during the first 2 min. of reperfusion but progressively decreases within 2 to 3 hours after reperfusion, resulting in a 2-fold increase of MVO. ${ }^{35,38}$ A further increase in MVO size has been demonstrated up to 48 hours after initiation of reperfusion. ${ }^{39}$ This progression of MVO is presumably related to reperfusion injury. Therefore, therapeutic interventions trying to attenuate MVO are likely to be most successful when initiated early after reperfusion. 


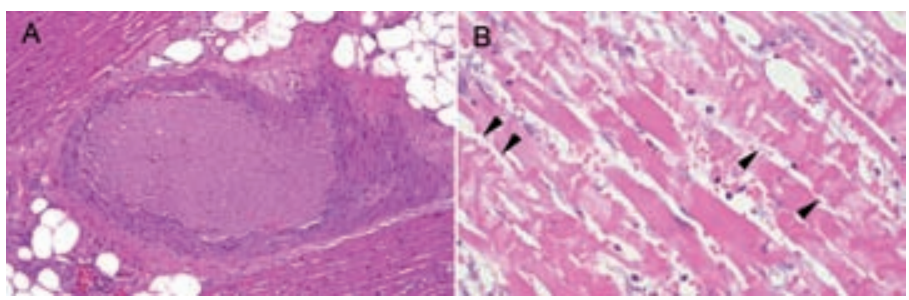

Figure 5. Obstructed microvessels and myocardial reperfusion injury. Distal platelet-rich (intramyocardial) embolus (A) when treated with thrombolytic therapy will result in reperfusion injury with (B) contraction band necrosis (arrowheads) and diffuse neutrophil and red cell infiltration in a patient with myocardial infarction secondary to epicardial coronary plaque rupture.
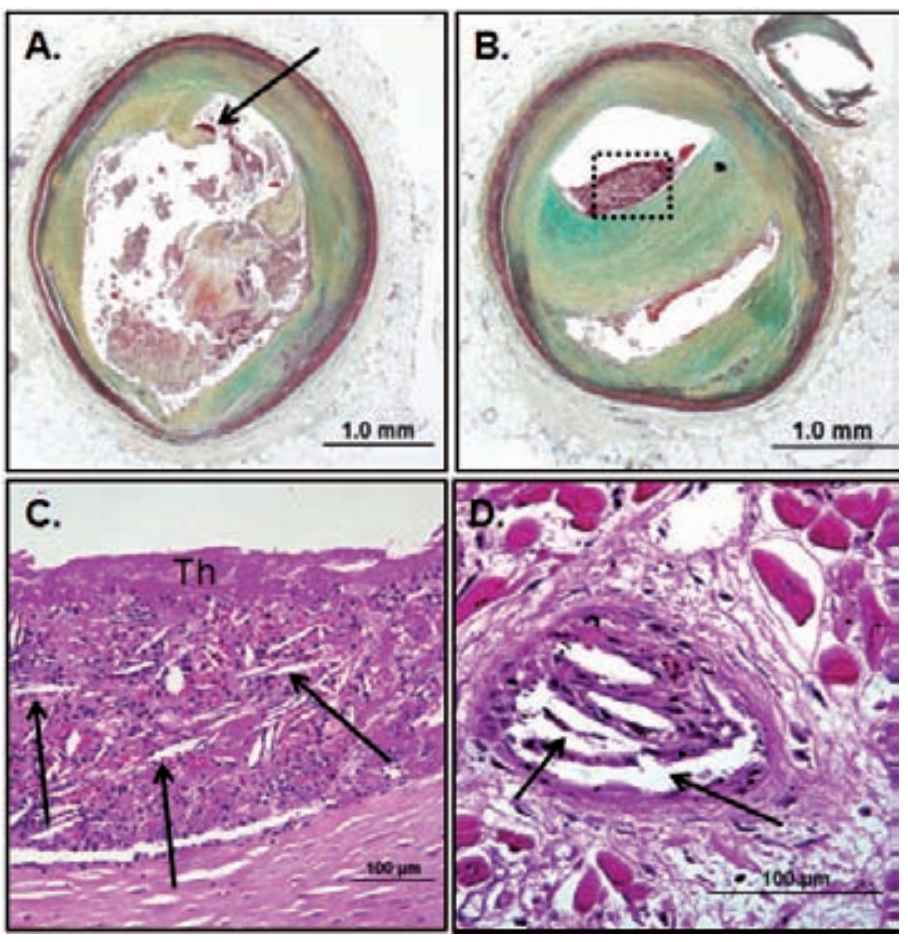

Figure 6. Representative micrographs of distal microembolization of plaque material from ruptured atheroma. (A) Low-power (Movat's pentachrome) view of rupture site (arrow) showing luminal fibrin and cholesterol clefts. (B) Adjacent distal segment to rupture site demonstrates nonocclusive thrombus composed of necrotic core material. (C) High-power image demonstrating cholesterol clefts (arrows) with superimposed thrombus (Th) (hematoxylin and eosin). (D) High-power image of intramural coronary artery atheroembolus consisting of cholesterol clefts (arrows) and surrounded by healing myocardium in a patient who underwent percutaneous coronary intervention for acute myocardial infarction. 


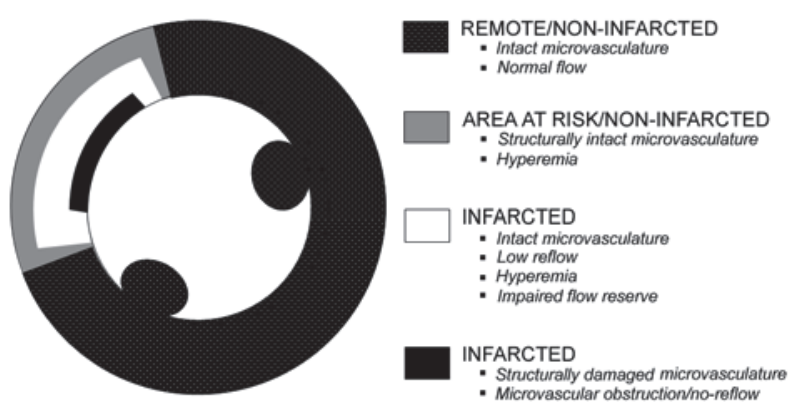

Figure 7. Different regions of microvascular flow after re-perfusion. Schematic short-axis presentation showing the different regions of microvascular flow in reperfused acute myocardial infarction.

\section{Diagnosis of MVO}

\section{Coronary angiography}

After the recognition that complete thrombotic coronary occlusion caused AMI and that early reperfusion with thrombolytic agents increased myocardial salvage and reduced mortality, there was a need to angiographically assess the quality of reperfusion. ${ }^{40}$ Successful reperfusion is defined by assessing antegrade angiographic epicardial blood flow, graded as TIMI flow; TIMI flow grade 0: absent antegrade flow; TIMI flow grade 1: partial contrast penetration beyond an occlusion with incomplete distal filling; TIMI flow grade 2: patent epicardial artery with opacification of the entire distal artery (however, contrast filling or washout is delayed); TIMI flow grade 3: patent epicardial artery with normal flow.

Early studies using this angiographic assessment found that mortality was significantly lower in patients with TIMI flow grade 3 than in those with other TIMI flow grades ( $4 \%$ vs. $8 \%, p<0.01$ ), and importantly, TIMI flow grade 2 was not associated with a significant survival advantage compared with TIMI flow grades 0 or $1 .{ }^{41} \mathrm{~A}$ TIMI flow grade $\leq 2$ without a residual obstructive lesion has been generally regarded as the "angiographic no reflow". 42 Although angiographic assessment of reperfusion is still useful in daily clinical practice, the clinician should be aware that TIMI flow grade 3 is not always synonymous with optimal tissue reperfusion, because MVO can be demonstrated in a substantial number of these patients. ${ }^{2}$

Because the TIMI flow grading system is semi-quantitative and subjective, a more objective angiographic marker of tissue perfusion was developed. The corrected TIMI frame count (CTFC) assesses the number of cine frames required for contrast to first reach standardized distal coronary landmarks in the culprit artery. A higher CTFC and thus slower epicardial flow $90 \mathrm{~min}$. after administration of a 
thrombolytic agent is associated with increased mortality and composite end points and adds prognostic information even in patients with TIMI flow grade $3 .{ }^{43}$

\section{Coronary blood flow velocity patterns}

In patients with substantial MVO the coronary blood flow velocity pattern is characterized by a reduction in systolic antegrade flow, the appearance of abnormal retrograde flow in early systole $(>10 \mathrm{~cm} / \mathrm{s})$, and a rapid deceleration time of diastolic flow velocity (<600 ms), which was found to be highly associated with TIMI flow grade 2 . 44,45

These distinct coronary flow velocity patterns are associated with a decreased recovery of LV function over time and seem accurate predictors of long-term cardiac events in reperfused AMI. ${ }^{46}$ These altered coronary blood flow velocity patterns are believed to be related to increased microvascular impedance as a consequence of profound microvascular damage and obstruction.

\section{Myocardial blush grading}

The assessment of TIMI flow grade and CTFC and the identification of distinct coronary flow velocity patterns only indirectly reflect the status of the microcirculation. Perfusion can also be assessed angiographically with a semi-quantitative description of myocardial contrast density on the final angiogram after reperfusion, described as TIMI myocardial perfusion (TMP) or myocardial blush grade. ${ }^{47,48}$ The TMP flow grades are defined as follows: TMP flow grade 0: failure of dye to enter the microvasculature; TMP flow grade 1: dye slowly enters but fails to exit the microvasculature; TMP flow grade 2: delayed entry and exit of dye from the microvasculature; TMP flow grade 3: normal entry and exit of dye from the microvasculature.

Myocardial perfusion grading also permits risk stratification and adds prognostic information even in patients with TIMI flow grade 3. Achievement of both TIMI flow grade 3 and myocardial blush grade 3 is associated with mortality under $1 \% .{ }^{49}$

Previous studies have shown that $11 \%$ of patients who had established epicardial patency and TIMI flow grade 3 had a myocardial blush grade of $0 / 1$, again emphasizing that TIMI flow grading is an inaccurate marker of tissue reperfusion. ${ }^{50}$ It is believed, however, that myocardial blush only indirectly reflects microvascular status but predominantly describes varying degrees of extravasation and washout of dye that is caused by a combination of increased capillary permeability, capillary resistance, and interstitial edema. ${ }^{51}$

The usefulness of angiographic and coronary blood flow velocity methods to detect MVO is disputable, because they are often performed immediately after mechanical reperfusion, a time point where microvascular flow is known to be hyperemic, and therefore they might not be an accurate reflection of the final mi- 
crovascular damage. Experimental studies have shown that the size of MVO increases depending on the time after reperfusion as a result of reperfusion injury. ${ }^{35}$

\section{Myocardial contrast echocardiography}

Myocardial contrast echocardiography (MCE) uses small microbubbles $(<5 \mu \mathrm{m})$ that possess an intravascular rheology similar to that of red blood cells and remain entirely within the microvasculature. During constant infusion, a steady state is achieved after approximately 2 to $3 \mathrm{~min}$. After destruction with high energy pulses, the rate of microbubble replenishment is proportionate to blood flow. Myocardial contrast echocardiography is able to delineate the area-at-risk for necrosis during acute coronary occlusion and has gained tremendous insight into post-ischemic tissue reperfusion. ${ }^{52}$ A persistent contrast defect despite a patent epicardial artery after reperfusion correlates with MVO. ${ }^{2}$

Myocardial contrast echocardiography studies also made clear that substantial MVO could be observed in all patients with TIMI flow grade 2 and in $16 \%$ of patients with TIMI flow grade $3 .{ }^{53}$

Myocardial contrast echocardiography predicted segmental myocardial recovery with a sensitivity of $88 \%$ and a specificity of $74 \%$ and a positive and negative predictive value of $83 \%$ and $81 \%$, respectively. ${ }^{54}$ In addition, MCE studies have shown that pericardial effusion, early congestive heart failure, and adverse arrhythmias occur significantly more frequently in patients with MVO. ${ }^{55}$

The technique of intracoronary injection of microbubbles before and shortly after coronary reflow make it less universally applicable, and although intravenous methods have become available, several disadvantages to the use of MCE remain: moderate spatial resolution, operator dependency, incomplete LV coverage with suboptimal visualization of the lateral wall, and semi-quantitative assessment of MVO. ${ }^{54,56}$

\section{Cardiovascular magnetic resonance}

Two contrast (gadolinium)-enhanced cardiovascular MRI (CMR) techniques are used to detect MVO in AMI. The primary technique is first-pass perfusion cardiovascular MRI (FPP-CMR). ${ }^{7,57,58}$ With this technique, multiple imaging planes are dynamically imaged to track an intravenous bolus of contrast into the myocardium. A homogenous increase in signal intensity in normal and infarcted myocardium is observed early after contrast administration, whereas MVO is seen as an area of reduced signal intensity (hypoenhancement) in the central core of the infarct that persists for $>2$ min. after contrast administration (early MVO).

The second technique is delayed enhancemant (DE-) CMR. ${ }^{5,59,60}$ Due to slow washout and local accumulation of contrast 10 to $15 \mathrm{~min}$. after contrast injection, 
the infarcted myocardium appears hyperenhanced or "bright" compared with normal noninfarcted myocardium. ${ }^{61}$ This hyperenhanced region closely corresponds to the area of necrosis as measured by triphenyltetrazolium chloride staining. ${ }^{62} \mathrm{~A}$ central hypoenhanced region within the hyperenhanced region corresponds to MVO (late MVO).

FPP-CMR was found to be more sensitive for the detection of MVO compared with DE-CMR. ${ }^{59}$ Experimental studies have demonstrated that the spatial extent as well as location of early MVO closely correlates with histopathological analyses. ${ }^{36,63}$

DE-CMR, as compared with FPP-CMR, underestimates the extent of MVO and might miss small regions of MVO due to slow penetration of contrast into the MVO region over time. ${ }^{64}$ FPP-CMR is hampered by incomplete LV coverage, low signal-tonoise ratio, and low spatial resolution interfering with MVO detection. Imaging protocols that combine early imaging and complete LV coverage might overcome this. ${ }^{65}$ Not only the timing after contrast administration but also the timing of imaging after AMI is important, because only persistent MVO after 1 week independently predicted adverse LV remodeling, unlike MVO that was present at 2 days but disappeared after 1 week. ${ }^{58}$

In contrast to MCE, cardiovascular MRI allows multislice imaging with high tissue contrast and high spatial resolution, enabling accurate quantification and localization of MVO and infarct size relative to the entire LV. Only one experimental study directly compared MCE and FPP-CMR with thioflavin-S staining and found MCE to overestimate and FPP-CMR to underestimate MVO size. This can be explained by the predominantly intravascular sojourn of microbubbles, whereas gadolinium as a freely diffusible tracer rapidly extravasates into the interstitium, thereby artificially decreasing the area of MVO. ${ }^{63}$ In addition, microbubbles are larger than gadolinium molecules and might become temporarily plugged in microvessels that would allow passage of gadolinium.

Cardiovascular MRI studies have shown that the presence of MVO significantly increases with infarct transmurality and independently predicts adverse events, including adverse LV remodeling, congestive heart failure, and death. ${ }^{7,55}$ In a recent study, the extent of MVO was not found to be related to the change in LVEF at follow-up, suggesting that MVO size might be clinically less important than its mere presence. ${ }^{66}$

\section{ECG}

In patients treated with thrombolytic therapy, complete STR $(\geq 70 \%)$ is a highly accurate predictor of infarct-related artery (IRA) patency and is associated with lower mortality. ${ }^{67-69}$ However, up to $50 \%$ of patients with absence of complete STR (STR $<70 \%$ ) might still have a patent IRA and TIMI flow grade 3. ${ }^{68,70}$ After successful primary $\mathrm{PCl}$ and establishment of TIMI flow grade 3, 30\% to $50 \%$ of patients have 
incomplete STR, indicating that microcirculatory perfusion might be impaired. ${ }^{71,72}$ Only a limited number of studies have directly related incomplete STR to MVO by coronary Doppler flow velocity measurements, cardiovascular MRI, and MCE. ${ }^{66,73,74}$ The interpretation of STR data should be done cautiously, because methodologies, definitions, and time points of measuring STR differ between studies.

Most studies have used the sum of STR method from standard 12-lead ECG, in which ST-segment deviation in all leads is summed and compared before and after intervention. Single lead STR $\geq 50 \%$ in the lead with maximum initial ST-segment elevation is a simple, universally available alternative that predicts TIMI flow grade 3 with $70 \%$ sensitivity and $54 \%$ specificity. ${ }^{75}$ Single lead STR is comparably accurate in predicting outcome as sum STR measurements. ${ }^{76}$ Minimal absolute ST-segment deviation after thrombolytic therapy can also be used to predict IRA patency. ${ }^{77}$ Because ST-segment elevation and STR can behave dynamically over time, continuous instead of serial ECG assessment might provide additional information on epicardial patency and quality of myocardial reperfusion. ${ }^{78}$

Other electrocardiographic changes have been described as signs of successful angiographic reperfusion (TIMI flow grades 2 or 3). Terminal T-wave inversion was observed in $60 \%$ of patients, and a patent coronary artery was associated with an increased number of accelerated idioventricular rhythms in small studies. ${ }^{79-81}$ These markers, however, only reflect IRA patency and have not been validated against other measures of MVO.

\section{Management of no-reflow}

Despite major advances in the understanding of the underlying pathophysiology of MVO, many different mechanical and pharmacologic therapeutic approaches to prevent or attenuate MVO have revealed very different and, in part, contradictory results. Interventions that were effective in the experimental setting did not show significant effects clinically, and vice versa.

The use of a microcirculatory protection device effectively retrieved embolic debris during $\mathrm{PCl}$ in $\mathrm{AMI}$ but failed to improve microvascular flow, reduce infarct size, or result in enhanced event-free survival. ${ }^{32}$ Although the aspiration of thrombus significantly improved reperfusion compared with conventional $\mathrm{PCl}$, a substantial number of patients still experienced low myocardial blush grades and incomplete STR. ${ }^{82}$ Likewise, intra-aortic balloon counterpulsation significantly reduced MVO experimentally, but a recent meta-analysis of clinical studies using intra-aortic balloon counterpulsation in the setting of ST-segment elevation myocardial infarction suggested that this is not associated with a change in LVEF and increased survival at follow-up. ${ }^{83,84}$ 
A range of pharmacologic adjunctive measures of reperfusion have been evaluated. Glycoprotein Ilb/IIla inhibition significantly improved microvascular flow and reduced infarct size in a dog model of coronary occlusion/reperfusion and significantly increased the rate of complete STR, coronary artery patency, and CTFC in clinical trials. ${ }^{85-88}$ Possibly by reversing microvascular spasm, the administration of intracoronary verapamil after $\mathrm{PCl}$ decreased no-reflow zones leading to a better functional outcome in first AMI patients. ${ }^{89}$ The extent of MVO is proportional to the number of polymorphonuclear leukocytes present in the infarcted area. The inhibition of complement pathways decreased polymorphonuclear leukocyte free radical generation and enhanced the recovery of coronary flow in an isolated rat heart model. $^{90,91}$

Circulating levels of endothelin, a potent and long-acting vasoconstrictor, are low in normal individuals but increased during ischemia, with a further increase during reperfusion. ${ }^{16}$ Endothelin-mediated vasoconstriction, neutrophil plugging, and microvascular permeability play a role in the pathogenesis of MVO. The significant reduction of MVO and infarct size in a canine model after the administration of an endothelin A-receptor antagonist after $90 \mathrm{~min}$ of coronary occlusion supports this concept. ${ }^{92}$

Selective administration of intracoronary adenosine attenuated functional and structural abnormalities of the microvasculature, leading to improved regional ventricular function as compared with placebo in a canine model. ${ }^{93}$ In the clinical setting, however, although infarct size was reduced, a 3-hour infusion of adenosine in reperfused ST-segment elevation myocardial infarction patients failed to show an improved outcome in the AMISTAD II (Acute Myocardial Infarction STudy of ADenosine) trial. ${ }^{94}$

These discrepant results underscore the complex underlying mechanisms implicated in the pathophysiology of MVO and suggest that successful therapy should be multitargeted.

\section{Conclusions}

Temporary coronary occlusion followed by reperfusion results in microvascular damage-with endothelial swelling, neutrophil activation, and entrapment-leading to reperfusion-induced myocardial damage. Microvascular flow after reperfusion is spatially and temporally complex. Regions of hyperemia, impaired vasodilatory flow reserve, and very low flow coexist. The magnitude and spatial extent of these perfusion patterns vary over time as a result of reperfusion injury. Microvascular obstruction is independently associated with adverse LV remodeling and prognosis. Therefore, its detection is crucial. Several techniques (coronary angiography, MCE, cardiovascular MRI, ECG) measuring slightly different biological and functional parame- 
ters are used clinically and experimentally for the evaluation of MVO (Table 1). Currently, there is no consensus on how and when MVO should be evaluated after AMI.

Table 1. Clinical Methods to Detect MVO.

\begin{tabular}{|c|c|c|}
\hline Technique & Generally accepted as MVO & reference \\
\hline \multicolumn{3}{|l|}{ Invasive } \\
\hline \multicolumn{3}{|l|}{ Coronary angiography } \\
\hline TIMI flow & $\leq 2$ & $(42)$ \\
\hline Corrected TIMI Frame Count & $>40$ & (49) \\
\hline \multicolumn{3}{|l|}{ Coronary blood flow patterns } \\
\hline Systolic retrograde flow & $>10 \mathrm{~cm} / \mathrm{sec}$ & $(44,45)$ \\
\hline Diastolic flow velocity deceleration & $<600$ msec & $(44,45)$ \\
\hline \multicolumn{3}{|l|}{ Myocardial blush grade } \\
\hline TMP grade & $\leq 1$ & $(47,50)$ \\
\hline \multicolumn{3}{|l|}{ Non-invasive } \\
\hline$\overline{M C E}$ & Contrast defect within area at risk despite angi- & $(2,53)$ \\
\hline \multicolumn{3}{|l|}{$C M R$} \\
\hline Early contrast enhancement & Central hypoenhancement within the infarcted & $(7,57,58)$ \\
\hline Late contrast enhancement & Central hypoenhancement within the area of & $(5,59,60)$ \\
\hline \multicolumn{3}{|l|}{ ECG } \\
\hline STR (60-90 min after reperfusion) & $<30 \%$ & $(67,70,74)$ \\
\hline STD (90 min after reperfusion) & $1 \mathrm{~mm}$ for inferior $\mathrm{Ml}$ & $(77)$ \\
\hline
\end{tabular}

Abbreviations: $\mathrm{MVO}=$ microvascular obstruction; $\mathrm{TIMI}=$ Thrombolysis In Myocardial Infarction; TMP=TIMI myocardial perfusion; $\mathrm{MCE}=$ myocardial contrast echocardiography; $\mathrm{CMR}=$ cardiovascular magnetic resonance; FPP=first pass perfusion; $\mathrm{MI}=$ myocardial infarction; STR=ST segment resolution; STD=ST segment deviation. 


\section{References}

1. An international randomized trial comparing four thrombolytic strategies for acute myocardial infarction. The GUSTO investigators. N Engl J Med. Sep 2 1993;329(10):673-682.

2. Ito H, Tomooka T, Sakai N, et al. Lack of myocardial perfusion immediately after successful thrombolysis. A predictor of poor recovery of left ventricular function in anterior myocardial infarction. Circulation. May 1992;85(5):1699-1705.

3. Kloner RA, Ganote CE, Jennings RB. The "no-reflow" phenomenon after temporary coronary occlusion in the dog. J Clin Invest. Dec 1974;54(6):1496-1508.

4. Gerber BL, Rochitte CE, Melin JA, et al. Microvascular obstruction and left ventricular remodeling early after acute myocardial infarction. Circulation. Jun 13 2000;101(23):2734-2741.

5. Hombach V, Grebe O, Merkle N, et al. Sequelae of acute myocardial infarction regarding cardiac structure and function and their prognostic significance as assessed by magnetic resonance imaging. Eur Heart J. Mar 2005;26(6):549-557.

6. Piana RN, Paik GY, Moscucci M, et al. Incidence and treatment of 'no-reflow' after percutaneous coronary intervention. Circulation. Jun 1994;89(6):2514-2518.

7. Wu KC, Zerhouni EA, Judd RM, et al. Prognostic significance of microvascular obstruction by magnetic resonance imaging in patients with acute myocardial infarction. Circulation. Mar 3 1998;97(8):765-772.

8. Roe MT, Ohman EM, Maas AC, et al. Shifting the open-artery hypothesis downstream: the quest for optimal reperfusion. J Am Coll Cardiol. Jan 2001;37(1):9-18.

9. Yellon DM, Hausenloy DJ. Myocardial reperfusion injury. N Engl J Med. Sep 13 2007;357(11):11211135.

10. Buja LM. Myocardial ischemia and reperfusion injury. Cardiovasc Pathol. Jul-Aug 2005;14(4):170175.

11. Stanley WC. Cardiac energetics during ischaemia and the rationale for metabolic interventions. Coron Artery Dis. Feb 2001;12 Suppl 1:S3-7.

12. Reimer KA, Lowe JE, Rasmussen MM, et al. The wavefront phenomenon of ischemic cell death. 1. Myocardial infarct size vs duration of coronary occlusion in dogs. Circulation. Nov 1977;56(5):786794.

13. Jennings RB, Steenbergen C, Jr., Reimer KA. Myocardial ischemia and reperfusion. Monogr Pathol. 1995;37:47-80.

14. Kloner RA, Rude RE, Carlson N, et al. Ultrastructural evidence of microvascular damage and myocardial cell injury after coronary artery occlusion: which comes first? Circulation. Nov 1980;62(5):945952.

15. Reimer KA, Jennings RB. The changing anatomic reference base of evolving myocardial infarction. Underestimation of myocardial collateral blood flow and overestimation of experimental anatomic infarct size due to tissue edema, hemorrhage and acute inflammation. Circulation. Oct 1979;60(4):866-876.

16. Virmani R, Forman MB, Kolodgie FD. Myocardial reperfusion injury. Histopathological effects of perfluorochemical. Circulation. Mar 1990;81(3 Suppl):IV57-68.

17. Velasco CE, Turner M, Inagami T, et al. Reperfusion enhances the local release of endothelin after regional myocardial ischemia. Am Heart J. Sep 1994;128(3):441-451.

18. Piper HM, Garcia-Dorado D, Ovize M. A fresh look at reperfusion injury. Cardiovasc Res. May 1998;38(2):291-300.

19. Eeckhout E, Kern MJ. The coronary no-reflow phenomenon: a review of mechanisms and therapies. Eur Heart J. May 2001;22(9):729-739.

20. Maxwell SR, Lip GY. Reperfusion injury: a review of the pathophysiology, clinical manifestations and therapeutic options. Int J Cardiol. Jan 31 1997;58(2):95-117.

21. Braunwald E, Kloner RA. Myocardial reperfusion: a double-edged sword? J Clin Invest. Nov 1985;76(5):1713-1719. 
22. Engler RL, Dahlgren MD, Morris DD, et al. Role of leukocytes in response to acute myocardial ischemia and reflow in dogs. Am J Physiol. Aug 1986;251(2 Pt 2):H314-323.

23. Babior BM. The respiratory burst of phagocytes. J Clin Invest. Mar 1984;73(3):599-601.

24. Erbel R, Heusch G. Coronary microembolization. J Am Coll Cardiol. Jul 2000;36(1):22-24.

25. Kawano $\mathrm{H}, \mathrm{Hayashida} \mathrm{T}$, Ohtani $\mathrm{H}$, et al. Histopathological findings of the no-reflow phenomenon following coronary intervention for acute coronary syndrome. Int Heart J. Mar 2005;46(2):327-332.

26. Saber RS, Edwards WD, Bailey KR, et al. Coronary embolization after balloon angioplasty or thrombolytic therapy: an autopsy study of 32 cases. J Am Coll Cardiol. Nov 1 1993;22(5):1283-1288.

27. Davies MJ, Thomas AC, Knapman PA, et al. Intramyocardial platelet aggregation in patients with unstable angina suffering sudden ischemic cardiac death. Circulation. Mar 1986;73(3):418-427.

28. Falk E. Unstable angina with fatal outcome: dynamic coronary thrombosis leading to infarction and/or sudden death. Autopsy evidence of recurrent mural thrombosis with peripheral embolization culminating in total vascular occlusion. Circulation. Apr 1985;71(4):699-708.

29. Frink RJ, Rooney PA, Jr., Trowbridge JO, et al. Coronary thrombosis and platelet/fibrin microemboli in death associated with acute myocardial infarction. Br Heart J. Feb 1988;59(2):196-200.

30. Hong MK, Popma JJ, Pichard AD, et al. Clinical significance of distal embolization after transluminal extraction atherectomy in diffusely diseased saphenous vein grafts. Am Heart J. Jun 1994;127(6):1496-1503.

31. Kaplan BM, Benzuly KH, Kinn JW, et al. Treatment of no-reflow in degenerated saphenous vein graft interventions: comparison of intracoronary verapamil and nitroglycerin. Cathet Cardiovasc Diagn. Oct 1996;39(2):113-118.

32. Stone GW, Webb J, Cox DA, et al. Distal microcirculatory protection during percutaneous coronary intervention in acute ST-segment elevation myocardial infarction: a randomized controlled trial. Jama. Mar 2 2005;293(9):1063-1072.

33. Henriques JP, Zijlstra F, Ottervanger JP, et al. Incidence and clinical significance of distal embolization during primary angioplasty for acute myocardial infarction. Eur Heart J. Jul 2002;23(14):11121117.

34. Silva-Orrego $\mathrm{P}$, Colombo $\mathrm{P}$, Bigi $\mathrm{R}$, et al. Thrombus aspiration before primary angioplasty improves myocardial reperfusion in acute myocardial infarction: the DEAR-MI (Dethrombosis to Enhance Acute Reperfusion in Myocardial Infarction) study. J Am Coll Cardiol. Oct 17 2006;48(8):1552-1559.

35. Ambrosio G, Weisman HF, Mannisi JA, et al. Progressive impairment of regional myocardial perfusion after initial restoration of postischemic blood flow. Circulation. Dec 1989;80(6):1846-1861.

36. Judd RM, Lugo-Olivieri $\mathrm{CH}$, Arai M, et al. Physiological basis of myocardial contrast enhancement in fast magnetic resonance images of 2-day-old reperfused canine infarcts. Circulation. Oct 1 1995;92(7):1902-1910.

37. Villanueva FS. Myocardial contrast echocardiography in acute myocardial infarction. Am J Cardiol. Nov 18 2002;90(10A):38J-47J.

38. Reffelmann T, Kloner RA. Microvascular reperfusion injury: rapid expansion of anatomic no reflow during reperfusion in the rabbit. Am J Physiol Heart Circ Physiol. Sep 2002;283(3):H1099-1107.

39. Rochitte CE, Lima JA, Bluemke DA, et al. Magnitude and time course of microvascular obstruction and tissue injury after acute myocardial infarction. Circulation. Sep 8 1998;98(10):1006-1014.

40. Kennedy JW, Ritchie JL, Davis KB, et al. The western Washington randomized trial of intracoronary streptokinase in acute myocardial infarction. A 12-month follow-up report. N Engl J Med. Apr 25 1985;312(17):1073-1078.

41. Brener SJ, Moliterno DJ, Aylward PE, et al. Reperfusion after primary angioplasty for ST-elevation myocardial infarction: predictors of success and relationship to clinical outcomes in the APEX-AMI angiographic study. Eur Heart J. May 2008;29(9):1127-1135.

42. Morishima I, Sone T, Okumura K, et al. Angiographic no-reflow phenomenon as a predictor of adverse long-term outcome in patients treated with percutaneous transluminal coronary angioplasty for first acute myocardial infarction. J Am Coll Cardiol. Oct 2000;36(4):1202-1209. 
43. Gibson CM, Murphy SA, Rizzo MJ, et al. Relationship between TIMI frame count and clinical outcomes after thrombolytic administration. Thrombolysis In Myocardial Infarction (TIMI) Study Group. Circulation. Apr 20 1999;99(15):1945-1950.

44. Iwakura K, Ito H, Takiuchi S, et al. Alternation in the coronary blood flow velocity pattern in patients with no reflow and reperfused acute myocardial infarction. Circulation. Sep 15 1996;94(6):12691275.

45. Lepper W, Hoffmann R, Kamp O, et al. Assessment of myocardial reperfusion by intravenous myocardial contrast echocardiography and coronary flow reserve after primary percutaneous transluminal coronary angioplasty [correction of angiography] in patients with acute myocardial infarction. Circulation. May 23 2000;101(20):2368-2374.

46. Furber AP, Prunier F, Nguyen HC, et al. Coronary blood flow assessment after successful angioplasty for acute myocardial infarction predicts the risk of long-term cardiac events. Circulation. Dec 7 2004;110(23):3527-3533.

47. Gibson CM, Cannon CP, Murphy SA, et al. Relationship of TIMI myocardial perfusion grade to mortality after administration of thrombolytic drugs. Circulation. Jan 18 2000;101(2):125-130.

48. van 't Hof AW, Liem A, Suryapranata $H$, et al. Angiographic assessment of myocardial reperfusion in patients treated with primary angioplasty for acute myocardial infarction: myocardial blush grade. Zwolle Myocardial Infarction Study Group. Circulation. Jun 16 1998;97(23):2302-2306.

49. Gibson CM, Cannon CP, Murphy SA, et al. Relationship of the TIMI myocardial perfusion grades, flow grades, frame count, and percutaneous coronary intervention to long-term outcomes after thrombolytic administration in acute myocardial infarction. Circulation. Apr 23 2002;105(16):1909-1913.

50. Henriques JP, Zijlstra F, van 't Hof AW, et al. Angiographic assessment of reperfusion in acute myocardial infarction by myocardial blush grade. Circulation. Apr 29 2003;107(16):2115-2119.

51. Kaul S. Coronary angiography cannot be used to assess myocardial perfusion in patients undergoing reperfusion for acute myocardial infarction. Heart. Nov 2001;86(5):483-484.

52. Villanueva FS, Glasheen WP, Sklenar J, et al. Assessment of risk area during coronary occlusion and infarct size after reperfusion with myocardial contrast echocardiography using left and right atrial injections of contrast. Circulation. Aug 1993;88(2):596-604.

53. Ito H, Okamura A, Iwakura K, et al. Myocardial perfusion patterns related to thrombolysis in myocardial infarction perfusion grades after coronary angioplasty in patients with acute anterior wall myocardial infarction. Circulation. Jun 1 1996;93(11):1993-1999.

54. Greaves K, Dixon SR, Fejka M, et al. Myocardial contrast echocardiography is superior to other known modalities for assessing myocardial reperfusion after acute myocardial infarction. Heart. Feb 2003;89(2):139-144.

55. Ito H, Maruyama A, Iwakura K, et al. Clinical implications of the 'no reflow' phenomenon. A predictor of complications and left ventricular remodeling in reperfused anterior wall myocardial infarction. Circulation. Jan 15 1996;93(2):223-228.

56. Porter TR, Li S, Oster R, et al. The clinical implications of no reflow demonstrated with intravenous perfluorocarbon containing microbubbles following restoration of Thrombolysis In Myocardial Infarction (TIMI) 3 flow in patients with acute myocardial infarction. Am J Cardiol. Nov 15 1998;82(10):1173-1177.

57. Lima JA, Judd RM, Bazille A, et al. Regional heterogeneity of human myocardial infarcts demonstrated by contrast-enhanced MRI. Potential mechanisms. Circulation. Sep 1 1995;92(5):1117-1125.

58. Orn S, Manhenke C, Greve OJ, et al. Microvascular obstruction is a major determinant of infarct healing and subsequent left ventricular remodelling following primary percutaneous coronary intervention. Eur Heart J. Jun 62009.

59. Nijveldt R, Hofman MB, Hirsch A, et al. Assessment of microvascular obstruction and prediction of short-term remodeling after acute myocardial infarction: cardiac MR imaging study. Radiology. Feb 2009;250(2):363-370. 
60. Yan AT, Gibson CM, Larose E, et al. Characterization of microvascular dysfunction after acute myocardial infarction by cardiovascular magnetic resonance first-pass perfusion and late gadolinium enhancement imaging. J Cardiovasc Magn Reson. 2006;8(6):831-837.

61. Simonetti OP, Kim RJ, Fieno DS, et al. An improved MR imaging technique for the visualization of myocardial infarction. Radiology. Jan 2001;218(1):215-223.

62. Kim RJ, Fieno DS, Parrish TB, et al. Relationship of MRI delayed contrast enhancement to irreversible injury, infarct age, and contractile function. Circulation. Nov 9 1999;100(19):1992-2002.

63. Wu KC, Kim RJ, Bluemke DA, et al. Quantification and time course of microvascular obstruction by contrast-enhanced echocardiography and magnetic resonance imaging following acute myocardial infarction and reperfusion. J Am Coll Cardiol. Nov 15 1998;32(6):1756-1764.

64. Lund GK, Stork A, Saeed M, et al. Acute myocardial infarction: evaluation with first-pass enhancement and delayed enhancement MR imaging compared with 201TI SPECT imaging. Radiology. Jul 2004;232(1):49-57.

65. Bekkers SC, Backes WH, Kim RJ, et al. Detection and characteristics of microvascular obstruction in reperfused acute myocardial infarction using an optimized protocol for contrast-enhanced cardiovascular magnetic resonance imaging. Eur Radiol. Jul 92009.

66. Nijveldt R, Beek AM, Hirsch A, et al. Functional recovery after acute myocardial infarction: comparison between angiography, electrocardiography, and cardiovascular magnetic resonance measures of microvascular injury. J Am Coll Cardiol. Jul 15 2008;52(3):181-189.

67. Barbash GI, Roth A, Hod H, et al. Rapid resolution of ST elevation and prediction of clinical outcome in patients undergoing thrombolysis with alteplase (recombinant tissue-type plasminogen activator): results of the Israeli Study of Early Intervention in Myocardial Infarction. $\mathrm{Br}$ Heart J. Oct 1990;64(4):241-247.

68. de Lemos JA, Antman EM, Giugliano RP, et al. ST-segment resolution and infarct-related artery patency and flow after thrombolytic therapy. Thrombolysis in Myocardial Infarction (TIMI) 14 investigators. Am J Cardiol. Feb 1 2000;85(3):299-304.

69. Schroder R, Dissmann R, Bruggemann T, et al. Extent of early ST segment elevation resolution: a simple but strong predictor of outcome in patients with acute myocardial infarction. J Am Coll Cardiol. Aug 1994;24(2):384-391.

70. de Lemos JA, Braunwald E. ST segment resolution as a tool for assessing the efficacy of reperfusion therapy. J Am Coll Cardiol. Nov 1 2001;38(5):1283-1294.

71. Feldman LJ, Coste P, Furber A, et al. Incomplete resolution of ST-segment elevation is a marker of transient microcirculatory dysfunction after stenting for acute myocardial infarction. Circulation. Jun 3 2003;107(21):2684-2689.

72. van 't Hof AW, Liem A, de Boer MJ, et al. Clinical value of 12-lead electrocardiogram after successful reperfusion therapy for acute myocardial infarction. Zwolle Myocardial infarction Study Group. Lancet. Aug 30 1997;350(9078):615-619.

73. Bekkers SC, Lemmert ME, Schalla S, et al. ST Segment Resolution After Reperfusion Therapy for Acute Myocardial Infarction is Related to Microvascular Obstruction (Abstr). Circulation. 2007;116 Suppl II:II-425.

74. Santoro GM, Valenti R, Buonamici $\mathrm{P}$, et al. Relation between ST-segment changes and myocardial perfusion evaluated by myocardial contrast echocardiography in patients with acute myocardial infarction treated with direct angioplasty. Am J Cardiol. Oct 15 1998;82(8):932-937.

75. Syed MA, Borzak S, Asfour A, et al. Single lead ST-segment recovery: a simple, reliable measure of successful fibrinolysis after acute myocardial infarction. Am Heart J. Feb 2004;147(2):275-280.

76. Zeymer $\mathrm{U}$, Schroder $\mathrm{R}$, Tebbe $\mathrm{U}$, et al. Non-invasive detection of early infarct vessel patency by resolution of ST-segment elevation in patients with thrombolysis for acute myocardial infarction; results of the angiographic substudy of the Hirudin for Improvement of Thrombolysis (HIT)-4 trial. Eur Heart J. May 2001;22(9):769-775. 
77. Cooper HA, de Lemos JA, Morrow DA, et al. Minimal ST-segment deviation: a simple, noninvasive method for identifying patients with a patent infarction-related artery after fibrinolytic administration. Am Heart J. Nov 2002;144(5):790-795.

78. Krucoff MW, Johanson P, Baeza R, et al. Clinical utility of serial and continuous ST-segment recovery assessment in patients with acute ST-elevation myocardial infarction: assessing the dynamics of epicardial and myocardial reperfusion. Circulation. Dec 21 2004;110(25):e533-539.

79. Gorgels AP, Vos MA, Letsch IS, et al. Usefulness of the accelerated idioventricular rhythm as a marker for myocardial necrosis and reperfusion during thrombolytic therapy in acute myocardial infarction. Am J Cardiol. Feb 1 1988;61(4):231-235.

80. Gressin V, Gorgels A, Louvard Y, et al. ST-segment normalization time and ventricular arrhythmias as electrocardiographic markers of reperfusion during intravenous thrombolysis for acute myocardial infarction. Am J Cardiol. Jun 15 1993;71(16):1436-1439.

81. Wehrens XH, Doevendans PA, Ophuis TJ, et al. A comparison of electrocardiographic changes during reperfusion of acute myocardial infarction by thrombolysis or percutaneous transluminal coronary angioplasty. Am Heart J. Mar 2000;139(3):430-436.

82. Svilaas T, Vlaar PJ, van der Horst IC, et al. Thrombus aspiration during primary percutaneous coronary intervention. N Engl J Med. Feb 7 2008;358(6):557-567.

83. Amado LC, Kraitchman DL, Gerber BL, et al. Reduction of "no-reflow" phenomenon by intra-aortic balloon counterpulsation in a randomized magnetic resonance imaging experimental study. J Am Coll Cardiol. Apr 7 2004;43(7):1291-1298.

84. Sjauw KD, Engstrom AE, Vis MM, et al. A systematic review and meta-analysis of intra-aortic balloon pump therapy in ST-elevation myocardial infarction: should we change the guidelines? Eur Heart J. Feb 2009;30(4):459-468.

85. Antman EM, Giugliano RP, Gibson CM, et al. Abciximab facilitates the rate and extent of thrombolysis: results of the thrombolysis in myocardial infarction (TIMI) 14 trial. The TIMI 14 Investigators. Circulation. Jun 1 1999;99(21):2720-2732.

86. de Lemos JA, Antman EM, Gibson CM, et al. Abciximab improves both epicardial flow and myocardial reperfusion in ST-elevation myocardial infarction. Observations from the TIMI 14 trial. Circulation. Jan 25 2000;101(3):239-243.

87. Kunichika H, Ben-Yehuda O, Lafitte $\mathrm{S}$, et al. Effects of glycoprotein Ilb/IIla inhibition on microvascular flow after coronary reperfusion. A quantitative myocardial contrast echocardiography study. J Am Coll Cardiol. Jan 21 2004;43(2):276-283.

88. Montalescot G, Barragan P, Wittenberg O, et al. Platelet glycoprotein IIb/IIla inhibition with coronary stenting for acute myocardial infarction. N Engl J Med. Jun 21 2001;344(25):1895-1903.

89. Taniyama $\mathrm{Y}$, Ito $\mathrm{H}$, Iwakura $\mathrm{K}$, et al. Beneficial effect of intracoronary verapamil on microvascular and myocardial salvage in patients with acute myocardial infarction. J Am Coll Cardiol. Nov 1 1997;30(5):1193-1199.

90. Prasad A, Stone GW, Stuckey TD, et al. Relation between leucocyte count, myonecrosis, myocardial perfusion, and outcomes following primary angioplasty. Am J Cardiol. Apr 15 2007;99(8):1067-1071.

91. Shandelya SM, Kuppusamy P, Herskowitz A, et al. Soluble complement receptor type 1 inhibits the complement pathway and prevents contractile failure in the postischemic heart. Evidence that complement activation is required for neutrophil-mediated reperfusion injury. Circulation. Dec 1993;88(6):2812-2826.

92. Galiuto L, DeMaria AN, del Balzo U, et al. Ischemia-reperfusion injury at the microvascular level: treatment by endothelin A-selective antagonist and evaluation by myocardial contrast echocardiography. Circulation. Dec 19 2000;102(25):3111-3116.

93. Babbitt DG, Virmani R, Forman MB. Intracoronary adenosine administered after reperfusion limits vascular injury after prolonged ischemia in the canine model. Circulation. Nov 1989;80(5):13881399. 
94. Ross AM, Gibbons RJ, Stone GW, et al. A randomized, double-blinded, placebo-controlled multicenter trial of adenosine as an adjunct to reperfusion in the treatment of acute myocardial infarction (AMISTAD-II). J Am Coll Cardiol. Jun 7 2005;45(11):1775-1780. 


\section{CHAPTER 3}

\section{Detection and characteristics of microvascular obstruction in reperfused acute myocardial infarction using an optimized protocol for contrast- enhanced cardiovascular magnetic resonance imaging}

Bekkers SCAM, Backes WH, Kim RJ, Snoep G, Gorgels APM, Lima Passos V, Waltenberger JL, Crijns HJGM, Schalla S 


\begin{abstract}
Several cardiovascular magnetic resonance imaging (CMR) techniques are used to detect microvascular obstruction (MVO) after acute myocardial infarction (AMI). To determine the prevalence of MVO and gain more insight into the dynamic changes in appearance of MVO, we studied 84 consecutive patients with a reperfused AMI on average 5 and 104 days after admission, using an optimized single breath-hold $3 \mathrm{D}$ inversion recovery gradient echo pulse sequence (IR-GRE) protocol. Early MVO ( 2 min post-contrast) was detected in 53 patients (63\%) and late MVO (10 min postcontrast) in 45 patients ( $54 \% ; p=0.008)$. The extent of MVO decreased from early to late imaging $(4.3 \pm 3.2 \%$ vs. $1.8 \pm 1.8 \%, p<0.001)$ and showed a heterogeneous pattern. At baseline, patients without MVO (early and late) had a higher left ventricular ejection fraction (LVEF) than patients with persistent late MVO (56 $\pm 7 \%$ vs. $48 \pm 7 \%$, $p<0.001$ ) and LVEF was intermediate in patients with early MVO but late MVO disappearance (54 $\pm 6 \%$ ). During follow-up, LVEF improved in all three subgroups but remained intermediate in patients with late MVO disappearance. This optimized single breath-hold 3D IR-GRE technique for imaging MVO early and late after contrast administration is fast, accurate and allows detection of patients with intermediate remodeling at follow-up.
\end{abstract}




\section{Introduction}

Reperfusion therapy in patients with acute myocardial infarction (AMI) salvages myocardium and reduces mortality. ${ }^{1-3}$ However, successful restoration of epicardial coronary artery patency after prolonged occlusion does not always lead to adequate reperfusion at the microvascular level. Previous studies have shown that the presence and absolute amount of microvascular obstruction (MVO) are associated with adverse left ventricular remodelling and prognosis. ${ }^{4-7}$ Microvascular flow after reperfusion is spatially and temporally complex, with coexisting regions of hyperemia, impaired vasodilatory flow reserve, low flow and no reflow. The magnitude and spatial extent of these perfusion patterns vary over time. ${ }^{8}$

MVO can be detected with cardiovascular magnetic resonance imaging (CMR) using the technique of first pass perfusion imaging (FPP) and traditional late contrast-enhanced CMR (late CE-CMR), but no consensus exists on which technique is more accurate. ${ }^{5,9-11}$ The importance of detecting MVO early after contrast injection is increasingly being recognized and has led to the development of additional CMR techniques. ${ }^{12,13}$ Whereas traditional late CE-CMR may underestimate the true extent of MVO as a result of diffusion of gadolinium into the area of MVO over time, FPP allows only incomplete coverage of the left ventricle and has a lower signal-tonoise ratio and spatial resolution. When the technique of traditional late CE-CMR is performed early after contrast administration (early CE-CMR), the advantages of complete ventricular coverage, optimal signal-to-noise ratio (SNR) and spatial resolution are combined and may overcome these shortcomings.

Optimal timing of imaging MVO and the dynamic changes of MVO over time in relation to clinical variables are not well known. In this study, a 3D inversion recovery pulse sequence was used early and late after contrast administration to determine the prevalence of MVO and gain more insight into the dynamic changes in appearance of MVO in relation to clinical variables. For early imaging the inversion time of the imaging sequence was first optimized in a pilot study.

\section{Materials and methods}

\section{Patients}

The study was approved by the institutional review board of our hospital, and patients were included after written informed consent was obtained. Between August 2006 and March 2008, 84 consecutive patients with a first ST segment elevation myocardial infarction (STEMI) referred to our institution for primary percutaneous coronary intervention ( $\mathrm{PCl}$ ) and presentation less than 12 hours after symptom onset were prospectively studied. Definition of myocardial infarction (MI) was based 
on the recent consensus document including appropriate rise and fall in cardiac biomarkers. ${ }^{14}$ Excluded were patients with claustrophobia, contraindications for CMR (cerebral clips, pacemakers, defibrillators, neurostimulators), severe congestive heart failure or cardiogenic shock (Killip class III and IV) at the time of CMR, atrial fibrillation, age below 18 years, severe renal failure (stage 4 or 5 ) and pregnancy.

Before emergency $\mathrm{PCl}$ all patients received aspirin $(500 \mathrm{mg})$, heparin (5,000 U) and clopidogrel $(600 \mathrm{mg})$. Use of thrombosuction and downstream administration of intravenous antiplatelet agents (abciximab), intracoronary nitroglycerine and adenosine were left to the discretion of the interventional cardiologist.

Cardiac biomarkers were sampled on admission, and at 6-, 12-, 18-, 24-, 36-, 48and 72-hour intervals. Area under the curve (AUC) biomarker levels were derived and expressed as 1,000 h.units $\mathrm{I}^{-1}$.

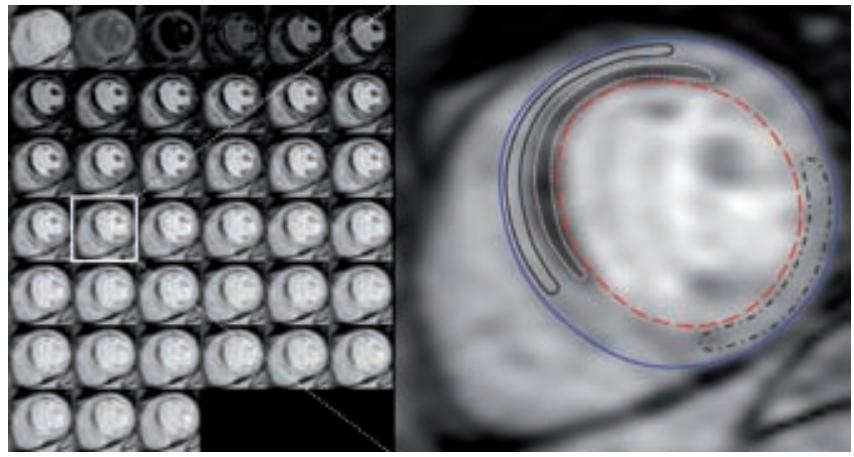

Figure 1. Look-Locker images (left panel) in a patient with regions of interest (ROIs) drawn in only one phase (right panel, enlarged from left panel). Left panel 39 phases of a single slice Look-Locker sequence in one of the eight patients. Right panel phase 20 is enlarged to demonstrate the different ROIs that were drawn in all phases: subendocardial hypoenhanced region (MVO) (dotted line), region directly adjacent to the hypoenhanced region (infarcted myocardium) (solid black line), region of noninfarcted (normal) myocardium (dotted-dashed line).

\section{CMR protocol}

Patients underwent CMR imaging $5 \pm 2$ days (range 2-10 days) and $104 \pm 11$ days (range 88-122 days) after admission. Images were acquired during multiple breathholds on a 1.5 Tesla MRI system (Philips Intera, Philips Medical Systems, Best, The Netherlands) with a cardiac software package and dedicated five-element phased array surface coil. ECG-gated cine images were obtained for functional analysis using a steady-state free precession sequence (slice thickness $6 \mathrm{~mm}$, slice gap $4 \mathrm{~mm}$, average repetition time (TR) and echo time (TE) 3.8/1.9 ms, respectively, flip angle $50^{\circ}$, FOV $350 \mathrm{~mm}$, matrix $256 \times 256$, typically $22-25$ phases per cardiac cycle), in the 
following orientations: single slice two-chamber long axis, three-chamber long axis, four-chamber long axis, and a short axis stack covering the left ventricle (LV) from the base to apex (typically 10-14 slices).

Subsequently, a single breath-hold (15-20 s) 3D inversion recovery gradient echo sequence completely covering the LV was used for early and late CE-CMR (acquired slice thickness $12 \mathrm{~mm}$, reconstructed slice thickness $6 \mathrm{~mm}$, average TR/TE 3.9/2.4 ms, multishot (50 profiles/shot) segmented partial echo readout every heartbeat (TFE), flip angle $15^{\circ}$, FOV $400 \mathrm{~mm}$, matrix $256 \times 256$, acquired and reconstructed pixel size $1.56 \times 1.56 \mathrm{~mm}$, typically $16-18$ slices). The pulse sequence was run at 2 and $10 \mathrm{~min}$ after intravenous administration of $0.2 \mathrm{mmol} / \mathrm{kg}$ body weight gadolinium diethylenetriaminepentaacetic acid (Gd-DTPA, Magnevist ${ }^{\circledR}$, Schering, Germany; injection rate $3 \mathrm{ml} / \mathrm{s}$ ). For early CE-CMR, the inversion time was first optimized in a pilot study:
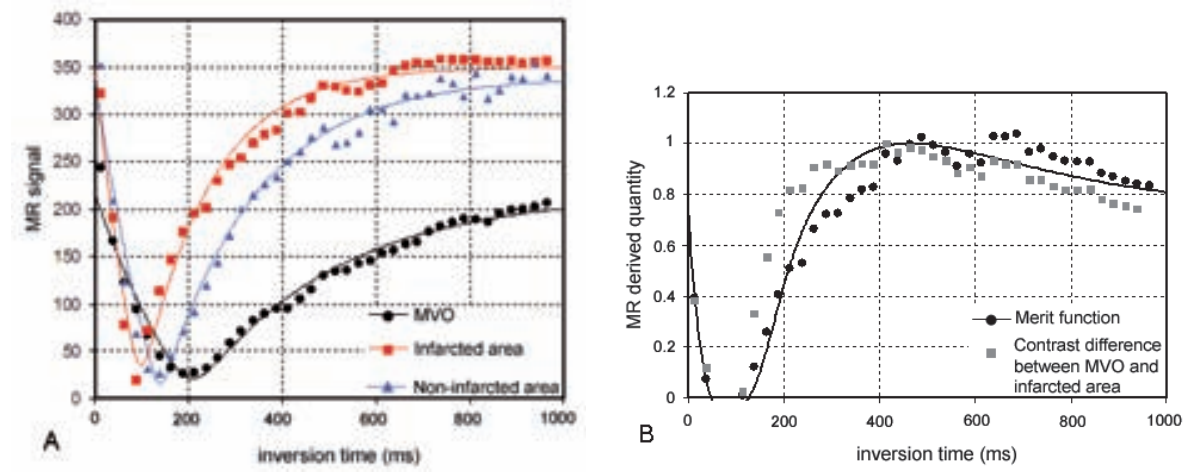

Figure 2. Signal intensity curves obtained from the Look-Locker sequence (A) and merit function of contrast and SNR. The MR-derived quantity was normalized to the maximum value (B). (A) Signal intensity (SI) curves in the region of microvascular obstruction (MVO), infarcted and noninfarcted myocardium obtained from Look-Locker inversion recovery images in a representative patient. Maximum contrast (i.e. SI difference) between MVO and infarcted myocardium would be obtained at an inversion time of approximately $340 \mathrm{~ms}$. b Combining optimised contrast and overall signal, the merit function provides a much less broader optimum at an inversion time of $465 \mathrm{~ms}$ for this case than for the contrast between MVO and infarcted area. Note the broad maximum in (B) and the relatively low signal at $340 \mathrm{~ms}$ (i.e. maximal SI difference in this case) in (A).

\section{Pilot study to find optimal TI for early CE-CMR}

We set out to find an optimal inversion time (TI) for early CE-CMR. For this, LookLocker images acquired 2 min after intravenous administration of gadolinium $(0.2 \mathrm{mmol} / \mathrm{kg}$ body weight; injection rate $3 \mathrm{ml} / \mathrm{s})$ were quantitatively analyzed in eight separate patients. This Look-Locker gradient echo (GRE) pulse sequence (slice thickness $10 \mathrm{~mm}$, average TR/TE 3.6/1.7 ms, multishot (3 profiles/shot) segmented full echo readout every heartbeat, flip angle $8^{\circ}$, FOV $370 \mathrm{~mm}$, resolution $256 \times 256$, 
39 phases, phase interval $15 \mathrm{~ms}$ ) was applied at the short axis level with the most pronounced wall motion abnormalities. For analysis, epicardial and endocardial contours were manually drawn in each phase. Additional regions of interest (ROIs) were defined in: (1) a subendocardial hypoenhanced region (MVO), (2) a region adjacent to the hypoenhanced region (infarcted myocardium) and (3) noninfarcted (normal) myocardium (Figure 1). All contours and ROls were carefully adjusted for cardiac motion as a result of contraction and breathing. The signal intensities (SI) of $\mathrm{MVO}$, infarcted and noninfarcted myocardium were determined as a function of the Look-Locker TI (Figure 2A). Measured inversion recovery SI curves were mathematically fitted to the inversion recovery magnetization function, which provides estimates of the T1 relaxation time of these tissue regions ${ }^{15}$. T1 parameter estimations were calculated in the MATLAB (The MathWorks, Natick, MA) programming environment using a Levenberg-Marquardt nonlinear fitting algorithm. As the implemented Look-Locker pulse sequences provided magnitude images, the Rician noise distribution was taken into account. ${ }^{16}$

Subsequently, the fitted SI curves of MVO and infarcted myocardium were subtracted to find the TI of maximum contrast, where contrast was defined as the difference in signal intensity (SI) between MVO and adjacent infarcted myocardium:

$$
\Delta \mathrm{SI}(\mathrm{TI})=\mathrm{SI}_{\text {infarct }}(\mathrm{TI})-\mathrm{SI}_{M v O}(\mathrm{TI})
$$

The TI for which this contrast between MVO and adjacent infarcted myocardium was maximal would at first sight provide the optimal TI for early CE-CMR (Figure 2A). However, a broad range of $\mathrm{TI}$ values (approximately 300-700 ms) were found with suboptimal overall signal characteristics of normal and infarcted myocardium.

It was beneficial to use higher TI values, because higher signal levels will be acquired at nearly constant contrast, but with improved endocardial and epicardial border definition. This observation led us to define a merit function (MF), which was defined as the product of the signal difference between MVO and infarcted myocardium multiplied by the signal intensity of noninfarcted myocardium ( $\mathrm{SI}_{\text {normal }}$ ) (Figure $2 \mathrm{~B})$ :

$$
\mathrm{MF}(\mathrm{TI})=\left[\mathrm{SI}_{\text {infarct }}(\mathrm{TI})-\mathrm{SI}_{M v O}(\mathrm{TI})\right] \times \mathrm{SI}_{\text {normal }}
$$

With this approach, both contrast and signal characteristics were simultaneously optimized. The merit function had a more narrow range of optimal TI values. The TI value of $530 \mathrm{~ms}$ ( $528 \pm 201 \mathrm{~ms}$ ), for which this merit function has its maximum, was used for subsequent early CE-CMR. 


\section{Early CE-CMR}

With the inversion time ( $\mathrm{TI}$ ) set to $530 \mathrm{~ms}$, images showed increased signal intensity of the blood pool in the ventricular lumen, infarcted and noninfarcted myocardium. MVO was defined as a central hypoenhanced region within the area of the infarctrelated artery (Figure 3, left panel).

\section{Late CE-CMR}

Based on the results of a Look-Locker sequence run at 10 min after contrast injection ( 8 min after early CE-CMR), images were then acquired with an individually set inversion time (typical range 200-280 ms) to optimally "null" noninfarcted myocardium. MVO was defined as a persistent central hypoenhanced area within the hyperenhanced area (Figure 3, right panel).

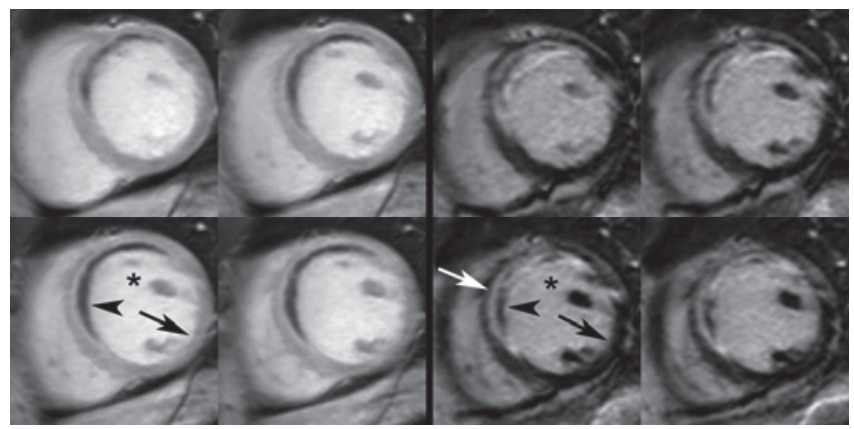

Figure 3. Early (left panel) and late CE-CMR images (right panel) in one of the patients. Two sets of four contiguous short axis slices at midlevel of the LV in a patient 5 days after reperfused acute anteroseptal wall myocardial infarction. Left panel 2 min after contrast injection: the blood pool $\left(^{*}\right)$ and myocardium show increased signal intensity (arrow), except for a central dark anteroseptal rim (arrowhead; early MVO). Right panel late hyper- and hypoenhancement: 10 min after contrast injection, signal from noninfarcted myocardium is "nulled" (black arrow) and the infarcted myocardium is hyperenhanced (white arrow). A dark central rim is observed within the bright area (late MVO; arrowhead).

\section{CMR image analysis}

CMR images were analyzed off-line using commercially available software (CAAS MRV 3.0, Pie Medical Imaging, Maastricht, the Netherlands) blinded to clinical information. Endocardial and epicardial contours were manually traced in enddiastolic and end-systolic short axis slices to determine left ventricular end-diastolic volume (LVEDV) and left ventricular end-systolic volume (LVESV), left ventricular 
ejection fraction (LVEF), and left ventricular end-diastolic mass and were indexed to body surface area (BSA). Endocardial and epicardial contours were manually traced on both early and late CE-CMR images. The presence or absence of MVO was determined independently by two experienced observers. Infarct size was measured on the late CE-CMR images by manual planimetry of hyperenhanced areas (including central hypoenhancement if present) and expressed as a percentage of LV mass.

\section{Statistical analysis}

Continuous data are expressed as mean \pm SD or as median and range when nonnormally distributed. Categorical data are expressed as frequencies with percentages. Differences between patients with and without early MVO were tested using an unpaired $t$ test. Spearman's rank correlation coefficient $(r)$ was used to determine correlations. The differences between proportions of paired categorical data were analyzed using McNemar's test. All images were independently analyzed by two observers blinded to the clinical data to determine the presence or absence of MVO, both for early and late. The interobserver agreement between readers was analyzed by using kappa $(\kappa)$ statistics. The same statistics was used to check agreement between early and late MVO by one observer only. Unless otherwise stated, a two-tailed $p$-value less than 0.05 was considered statistically significant.

Depending on the MVO status, patients were classified into three groups: those without MVO on early and late CE-CMR (absence), those with MVO on both early and late CE-CMR (persistence), and those with MVO on early but not late CE-CMR (disappearance). Comparisons among these groups were conducted either with the parametric one-way ANOVA or the nonparametric Kruskal-Wallis test for continuous variables. Post hoc pairwise comparisons were Bonferroni corrected. For categorical variables Chi-squared or Fisher's exact tests were applied. Logistic univariable regression models were fitted to investigate which variables individually were significantly associated with presence of MVO (persistence or disappearance). Subsequently, only significant predictors (at a 0.1 significance level) were added to a multivariate model to estimate their independent/adjusted effects. Similarly, univariable logistic regression followed by multivariable analyses were conducted to detect independent predictors of MVO disappearance.

A paired sample $t$ test was used to investigate whether LVEDV index and LVEF significantly changed between baseline and follow-up measurements. Statistical analysis was performed with SPSS software (version 15.0 for Windows; SPSS Inc., Chicago, Illinois). 


\section{Results}

The mean patient age was $60 \pm 11$ years and 61 (73\%) were men (Table 1 ). Most myocardial infarctions involved the right coronary artery $(55 \%)$, followed by the left anterior descending (32\%) and circumflex artery (13\%). More than half of the patients had single vessel disease (52\%). PCl with stenting was performed in 80 patients (95\%). In four patients no $\mathrm{PCl}$ was performed because of baseline thrombolysis in myocardial infarction (TIMI) 3 flow and a residual stenosis less than $30 \%$. Thrombosuction was performed in 26 patients (31\%), and intracoronary adenosine or nitroglycerine was given in 57 patients (68\%). Downstream abciximab was given in 43 patients (51\%). Final TIMI 3 flow was established in 75 patients (89\%) and TIMI 2 flow in the remaining 9 patients (11\%). The mean time from symptom onset to first balloon inflation or stent placement (total ischemic time) was $219 \pm 85 \mathrm{~min}$. Mean CK area under the curve (AUC) was $54 \pm 37\left(1,000 \mathrm{~h} \cdot \mathrm{units}^{-1}\right)$. Mean infarct size was $16 \pm 11 \%$ of the LV mass.

Table 1. Patient characteristics

\begin{tabular}{ll}
\hline Characteristic & Value \\
\hline Age (years) & $60 \pm 11$ \\
Male sex & $61(73)$ \\
BMI & $27 \pm 4$ \\
Smoking & $74(88)$ \\
Hypercholesterolaemia & $30(36)$ \\
Diabetes & $5(6)$ \\
Previous angina pectoris & $38(45)$ \\
Culprit & \\
$\quad$ RCA & $46(55)$ \\
$\quad$ LAD & $27(32)$ \\
$\quad$ Cx & $11(13)$ \\
Number of diseased vessels ${ }^{*}$ & \\
$\quad$ One & $44(52)$ \\
$\quad$ Two & $19(23)$ \\
$\quad$ Three & $21(25)$ \\
Thrombosuction & $26(31)$ \\
Time to balloon inflation (minutes) & $219 \pm 85$ \\
Adenosine or nitroglycerine i.c. & $57(68)$ \\
Abciximab downstream & $43(51)$ \\
Final TIMI 3 flow & $75(89)$ \\
CK (AUC, 1,000 h.units I ${ }^{-1}$ ) & $54 \pm 37$ \\
Infarct size (\% of LV mass) & $16 \pm 11$ \\
\hline
\end{tabular}

Values are presented as number of patients with percentages in parentheses or mean \pm standard deviation. Abbreviations: i.c.=intracoronary, AUC=area under the curve, $\mathrm{BMI}=$ body mass index, $\mathrm{RCA}=$ right coronary artery, $\mathrm{LAD}=$ left anterior descending artery, $\mathrm{Cx}=$ circumflex artery. * stenosis greater than $70 \%$. 


\section{Early CE-CMR}

MVO on early CE-CMR was observed in 53 patients (63\%). The extent of MVO was $4.3 \pm 3.2 \%$ of the LV mass; expressed as absolute values this was $7.8 \pm 6.4 \mathrm{~g}$. Compared with patients with absence of MVO, those with MVO had a higher left ventricular end-systolic volume index (LVESVi) $\left(45 \pm 13 \mathrm{ml} / \mathrm{m}^{2}\right.$ vs. $\left.37 \pm 11 \mathrm{ml} / \mathrm{m}^{2}, p=0.008\right)$, lower ejection fraction ( $49 \pm 7 \%$ vs. $56 \pm 7 \%, p<0.001)$, larger infarct size ( $21 \pm 9 \%$ vs. $6 \pm 8 \%, p<0.001)$ and higher CK AUC (68 \pm 32 vs. $\left.30 \pm 31\left(1,000 \mathrm{~h} \mathrm{U} \mathrm{I}^{-1}\right), p<0.001\right)$. Left ventricular end-diastolic volume index (LVEDVi) and LV mass tended to be higher in patients with MVO but did not reach statistical significance $\left(86 \pm 17 \mathrm{ml} / \mathrm{m}^{2} \mathrm{vs}\right.$. $81 \pm 14 \mathrm{ml} / \mathrm{m}^{2}, p=0.2$ and $64 \pm 12 \mathrm{~g} / \mathrm{m}^{2}$ vs. $59 \pm 10 \mathrm{~g} / \mathrm{m}^{2}, p=0.07$, respectively) .

In the multivariable analysis with variables from Table 1 , only infarct size remained an independent predictor of the presence of MVO (exp8 (OR) $1.2,95 \% \mathrm{Cl}$ $1.1-1.3, p<0.0001)$.

\section{Late CE-CMR}

MVO on late CE-CMR (persistence of MVO) was observed in 45 patients (54\%) compared with $53(63 \%)$ patients with MVO on early CE-CMR ( $p=0.008)$. Late MVO disappeared in eight patients (9\%) at the later time point. Late MVO was always found at the same location as early MVO (Figure 3). The agreement between early and late MVO ( $k=0.8, p<0.001$, Table 2 ) and the correlation between the extent of early and late MVO were good ( $r=0.8, p<0.0001$, Figure 4). The interobserver agreement for the presence or absence of early and late MVO was excellent ( $k$ value 0.9 for both early and late measurements, $p<0.001$ ).

Table 2. Agreement between early and late MVO

\begin{tabular}{llll}
\hline Early MVO & \multicolumn{2}{l}{ Late MVO } & \\
\cline { 2 - 4 } & Yes (\%) & No (\%) & Total (\%) \\
\hline Yes (\%) & $45(54)$ & $8(9)$ & $53(63)$ \\
No (\%) & 0 & $31(37)$ & $31(37)$ \\
Total (\%) & $45(54)$ & $39(46)$ & $84(100)$ \\
\hline
\end{tabular}

Values are presented as number of patients with percentages in parentheses. Good agreement was found between early and late MVO ( $\mathrm{k}=0.8$, $p<0.001)$. 

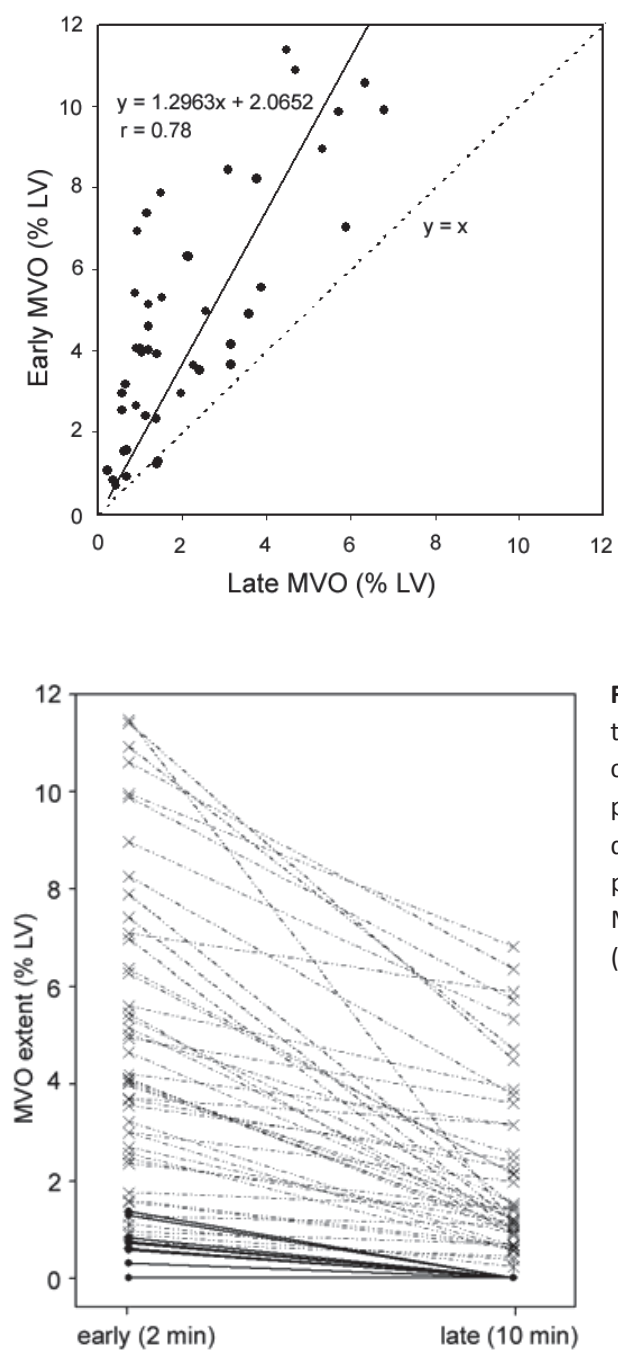

Figure 4. Correlation between early and late MVO. A good correlation was found between the extent of early and persistent late MVO. The average extent of early MVO was larger than that of persistent late MVO ( $r=0.78$, $p<0.001)$. The dotted line represents the line of identity and emphasizes the inequality of the measures.
Figure 5. Change in the extent of MVO. In all patients, the extent of early MVO decreased significantly but showed a heterogeneous pattern (MVO persistence $(\mathbf{x} \cdot \cdots \mathbf{x}))$. In eight patients early MVO

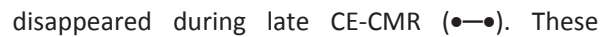
patients had a significantly smaller extent of early MVO than the patients with persistence of MVO $(0.8 \% \pm 0.4 \%$ vs. $5 \pm 3.1 \%, p<0.001)$.

\section{Early vs. late CE-CMR}

In all except one patient, the extent of MVO decreased significantly from early to late imaging $(4.3 \pm 3.2 \%$ vs. $1.8 \pm 1.8 \%, p<0.001$, Figures 3 and 5$)$. The median decrement in all patients was $66 \%$ (range 0-100\%); expressed as absolute amounts this resulted in a median decrement of $3.4 \mathrm{~g}$ (range $0-21 \mathrm{~g}$ ). The individual decrement was quite heterogeneous (Figure 5).

Thus, early MVO was absent in 31 (37\%) patients, persisted in 45 (54\%) patients and disappeared in $8(9 \%)$ patients during late CE-CMR. Most baseline characteristics 
were not found to be related to absence, persistence and disappearance of MVO (Table 3).

At baseline, infarct size and CK AUC were smallest and lowest in patients with absence of MVO, and largest for patients with persistence of late MVO (30 \pm 31 vs. $73 \pm 32\left(1,000 \mathrm{~h} \cdot\right.$ units $\left.^{-1}\right)$ and $6 \pm 8 \%$ vs. $23 \pm 9 \%, p<0.001$ for both). In addition, LVEF was highest in patients with absence of MVO, and lowest for patients with persistence of MVO (56 \pm 7 vs. $48 \pm 7 \%, p<0.001$ ).

The eight patients with disappearance of MVO had an infarct size and LVEF that was intermediate between those of patients with absence and persistence of MVO ( $12 \pm 4 \%$ and $54 \pm 6 \%$, respectively, Table 3 ). These eight patients had a significantly smaller extent of early MVO than patients with persistent late MVO $(0.8 \pm 0.4 \%$ vs. $5 \pm 3.1 \%, p<0.001$, Figure 5).

None of the clinical variables from Table 3 was found to be a significant predictor of MVO disappearance. From the CMR variables and after correction, only a smaller extent of early MVO remained independently associated with disappearance of late MVO (expB (OR) 0.034, 95\%Cl 0.001-0.908, $p<0.04)$.

\section{Follow-up CMR}

LVEDV index decreased and LVEF increased in all three subgroups as compared with baseline (Table 3). LVEF remained highest in patients without MVO as compared with patients with persistence of late MVO ( $59 \pm 6 \%$ vs. $51 \pm 7 \%, p<0.001)$. LVEF in patients with late MVO disappearance remained intermediate $(55 \pm 4 \%)$. 
Table 3. Patient characteristics in relation to MVO category (absence, disappearance and persistence)

\begin{tabular}{|c|c|c|c|c|}
\hline & \multicolumn{4}{|l|}{ Early MVO } \\
\hline & \multirow{2}{*}{$\begin{array}{l}\text { Absent } \\
(n=31)\end{array}$} & \multicolumn{2}{|l|}{ Present } & \multirow[b]{2}{*}{$p$-value } \\
\hline & & Disappearance & Persistence & \\
\hline Age (years) & $61 \pm 9$ & $61 \pm 10$ & $59 \pm 13$ & 0.8 \\
\hline Male sex & $22(71)$ & $5(63)$ & $34(76)$ & 0.7 \\
\hline BMI & $27 \pm 4$ & $26 \pm 2$ & $27 \pm 4$ & 0.7 \\
\hline Smoking & $26(84)$ & $7(88)$ & $41(91)$ & 0.6 \\
\hline Hypercholesterolaemia & $8(35)$ & $2(29)$ & $13(37)$ & 0.9 \\
\hline Diabetes & $3(10)$ & 0 & $2(4)$ & 0.4 \\
\hline Previous angina pectoris & $11(36)$ & $2(25)$ & $20(44)$ & 0.5 \\
\hline Anterior localisation of MI & $7(23)$ & $1(13)$ & $19(42)$ & 0.08 \\
\hline 3 vessel disease* & $8(26)$ & $1(13)$ & $12(27)$ & 0.7 \\
\hline Thrombosuction & $9(29)$ & $2(25)$ & $15(33)$ & 0.9 \\
\hline Time to balloon inflation (minutes) & $232 \pm 96$ & $196 \pm 80$ & $215 \pm 80$ & 0.6 \\
\hline Adenosine or nitroglycerine i.c. & $21(68)$ & $5(63)$ & $31(69)$ & 0.9 \\
\hline Abciximab downstream & $12(39)$ & $5(63)$ & $26(58)$ & 0.2 \\
\hline Final TIMI 3 flow & $27(90)$ & $5(71)$ & $41(91)$ & 0.3 \\
\hline Total CK (AUC, 1,000 h.units I ${ }^{-1}$ ) & $30 \pm 31^{\dagger}$ & $39 \pm 14^{\ddagger}$ & $73 \pm 32^{+, \neq}$ & $<0.001$ \\
\hline \multicolumn{5}{|l|}{ Baseline CMR } \\
\hline $\operatorname{LVEDV}_{\mathrm{i}}\left(\mathrm{ml} / \mathrm{m}^{2}\right)$ & $81 \pm 14$ & $87 \pm 17$ & $86 \pm 17$ & 0.2 \\
\hline $\operatorname{LVEF}(\%)$ & $56 \pm 7^{+}$ & $54 \pm 6$ & $48 \pm 7^{+}$ & $<0.001$ \\
\hline Infarct size (\% LV) & $6 \pm 8^{+}$ & $12 \pm 4^{\ddagger}$ & $23 \pm 9^{+, \neq}$ & $<0.001$ \\
\hline Early MVO size (\% of LV) & $0^{+}$ & $0.8 \pm 0.4$ & $5 \pm 3.1^{+}$ & $<0.001$ \\
\hline \multicolumn{5}{|l|}{ Follow-up CMR } \\
\hline $\operatorname{LVEDV}_{\mathrm{i}}\left(\mathrm{ml} / \mathrm{m}^{2}\right)$ & $76 \pm 17$ & $85 \pm 16$ & $84 \pm 20$ & 0.2 \\
\hline LVEF (\%) & $59 \pm 6^{\dagger}$ & $55 \pm 4$ & $51 \pm 7^{\dagger}$ & $<0.001$ \\
\hline
\end{tabular}

Values are presented as number of patients with percentages in parentheses or mean \pm standard deviation. Abbreviations: $\mathrm{BMI}=$ body mass index; $\mathrm{MI}=$ myocardial infarction; i.c.=intracoronary; $\mathrm{AUC}=\mathrm{area}$ under the curve; LVEDV $_{i}$ left ventricular end-diastolic volume index; LVEF left ventricular ejection fraction; $\mathrm{MVO}=$ microvascular obstruction. *Stenosis greater than $70 \%$. The superscripts $\left({ }^{+,} \neq\right)$indicate significant pairwise group comparison. 


\section{Discussion}

The findings of this study in 84 patients with a reperfused first acute STEMI can be summarized as follows. First, the prevalence of MVO early after contrast injection at $2 \mathrm{~min}$. (early MVO) is higher than with traditional late CE-CMR at $10 \mathrm{~min}$. (late MVO) using an optimized single breath-hold 3D inversion recovery gradient echo technique. Second, the extent of MVO decreases significantly depending on the time of imaging after contrast administration and this decrease is quite heterogeneous (Figures 3 and 5). Only the extent of early MVO is independently associated with persistence of late MVO. At baseline, patients with absence of MVO (early and late) have a significantly smaller infarct size and higher LVEF than patients with persistence of late MVO. In patients with early but late disappearance of MVO $(n=8)$, infarct size and LVEF are intermediate between those with absence and persistence of MVO and LVEF remains intermediate at follow-up.

The prevalence of MVO not only depends on the timing of imaging after infarction but also on the timing of imaging after contrast administration. ${ }^{9,17}$ In addition, it depends on the imaging technique used and as a consequence the reported prevalence of MVO has been inconsistent, ranging between 38 and $84 \%$ using FPP and between 28 and 36\% using traditional late enhancement imaging. ${ }^{9,18}$ Although the numbers were not greatly different, we found that the prevalence of MVO using early CE-CMR at 2 min after contrast administration was significantly higher compared with late CE-CMR. Early MVO completely filled in during late CE-CMR in $9 \%$ of patients.

Despite the importance of detecting MVO in AMI patients, a 'gold standard' for the clinical detection of MVO has not been established yet. Several techniques measuring slightly different biological parameters are currently used (TIMI flow, blush grade, ST segment resolution, myocardial contrast echocardiography and scintigraphy). In addition, different CMR techniques are being used such as traditional late CE-CMR, contrast-enhanced cine MR imaging, T2-weighted imaging and FPP, but the sensitivity is still undetermined. ${ }^{5,9-11,17,19-22}$

For the detection of early MVO, FPP seems sensitive because of its histopathological correlation to $\mathrm{MVO}$, as measured with thioflavin $\mathrm{S}$ staining and radioactive microsphere blood flow measurements in experimental studies. ${ }^{23} \mathrm{FPP}$, however, is hampered by incomplete left ventricular coverage, low SNR and low spatial resolution. Because of these shortcomings, other fast CMR techniques are increasingly being used for the detection of MVO early after contrast administration, including 3D T1-weighted inversion recovery gradient echo techniques. ${ }^{13,24}$ However, these studies did not specify the inversion times that were used for early imaging. Because we first optimized the inversion time for early imaging our approach allows imaging of the complete LV within a single breath-hold with good resolution, signal and contrast to noise. 
As we used the same pulse sequence for early and late CE-CMR, except for different inversion times, this enabled us to accurately evaluate the dynamic changes in appearance of MVO over time. In all but one patient the extent of MVO significantly decreased from early to late imaging. We found a great heterogeneity in the shrinkage of MVO over time, indicating that the prevalence of MVO is only one part of the larger underlying pathophysiology of MVO (Figures 3 and 5). For the first time we have described this heterogeneity in the shrinkage of MVO by using CMR. It likely represents the underlying heterogeneity in tissue perfusion, which was previously demonstrated in experimental studies. Negligible flow within the infarct core and progressively increasing flow towards the outer edges of the infarcted region are likely related to varying degrees of microvascular damage and obstruction. ${ }^{23,25,26}$ Whether this relates to varying degrees of remodeling over time or carries adverse prognostic information is currently unknown and needs to be studied further.

Our protocol enabled us to distinguish an additional group of patients with early but disappearance of late MVO. Recently, Nijveldt et al. compared three CMR techniques (early, intermediate and late hypoenhancement imaging) but did not specifically describe this subgroup. ${ }^{13}$ They found intermediate hypoenhancement in only two additional patients (3\%) compared with late hypoenhancement, whereas we observed this in eight additional patients (9\%). Although late hypoenhancement was the strongest predictor of LV remodeling over time in their study, we have shown that these patients have an LVEF that is intermediate at baseline and after followup. This may imply that these patients represent an intermediate risk group and should not be excluded from future therapies for MVO.

There are some limitations to our study. The inversion times used for the 3D inversion recovery sequence of both early and late CE-CMR were determined with a Look-Locker sequence. Although both sequences are gradient echo (GRE) readouts, the inherent difference in sequence parameters may cause differences in the amount of T1 weighting. It is therefore possible that the inversion times determined in our study are close but may be slightly out of kilter with the optimal inversion times for early and late CE-CMR. As the TI used in early CE-CMR was far above the "null" point for normal myocardium, small uncertainties in the optimal TI for early CE-CMR are not that critical as those for late CE-CMR. The optimal TI for late CECMR is traditionally set to "null" healthy myocardium 10-15 min. after contrast administration in each patient. This is not possible for early CE-CMR, as the 2-min. interval is too short for individual patients. Therefore, optimal TI for early CE-CMR was first determined in a pilot study.

The 2-min. interval was chosen in line with histopathological validation of MVO in earlier studies. ${ }^{17,23}$ With even earlier imaging (i.e. 30 sec.) sensitivity may increase but specificity may be decreased, because central hypoenhancement may appear in the setting of significant epicardial coronary disease even in the absence 
of myocardial infarction. Moreover, in our study we were interested in the dynamic change in appearance of MVO over time. Earlier imaging might have possibly shown a greater level of dynamic change. However, because most patients showed substantial shrinkage of MVO, the exact timing of early imaging is not expected to be that critical.

\section{Conclusion}

Using an optimized single breath-hold 3D inversion recovery gradient echo pulse sequence, the prevalence of MVO early after the injection of contrast medium is higher than with traditional late CE-CMR. Our results show that the appearance of MVO is dynamic and heterogeneous, and imaging MVO early and late after contrast administration may give more information about the severity and pathophysiology of MVO. In addition, this approach allows the discrimination of MVO subgroups that have different infarct sizes and remodeling parameters. 


\section{References}

1. An international randomized trial comparing four thrombolytic strategies for acute myocardial infarction. The GUSTO investigators. N Engl J Med. Sep 2 1993;329(10):673-682.

2. Serruys $P W$, Simoons ML, Suryapranata $H$, et al. Preservation of global and regional left ventricular function after early thrombolysis in acute myocardial infarction. I Am Coll Cardiol. Apr 1986;7(4):729-742.

3. Sheehan FH, Doerr R, Schmidt WG, et al. Early recovery of left ventricular function after thrombolytic therapy for acute myocardial infarction: an important determinant of survival. J Am Coll Cardiol. Aug 1988;12(2):289-300.

4. Gerber $\mathrm{BL}$, Rochitte $\mathrm{CE}$, Melin JA, et al. Microvascular obstruction and left ventricular remodeling early after acute myocardial infarction. Circulation. Jun 13 2000;101(23):2734-2741.

5. Hombach V, Grebe O, Merkle N, et al. Sequelae of acute myocardial infarction regarding cardiac structure and function and their prognostic significance as assessed by magnetic resonance imaging. Eur Heart J. Mar 2005;26(6):549-557.

6. Ito H, Tomooka T, Sakai N, et al. Lack of myocardial perfusion immediately after successful thrombolysis. A predictor of poor recovery of left ventricular function in anterior myocardial infarction. Circulation. May 1992;85(5):1699-1705.

7. Wu KC, Zerhouni EA, Judd RM, et al. Prognostic significance of microvascular obstruction by magnetic resonance imaging in patients with acute myocardial infarction. Circulation. Mar 3 1998;97(8):765-772.

8. Villanueva FS. Myocardial contrast echocardiography in acute myocardial infarction. Am J Cardiol. Nov 18 2002;90(10A):38J-47J.

9. Lund GK, Stork A, Saeed M, et al. Acute myocardial infarction: evaluation with first-pass enhancement and delayed enhancement MR imaging compared with 201TI SPECT imaging. Radiology. Jul 2004;232(1):49-57.

10. Taylor AJ, Al-Saadi N, Abdel-Aty $\mathrm{H}$, et al. Detection of acutely impaired microvascular reperfusion after infarct angioplasty with magnetic resonance imaging. Circulation. May 4 2004;109(17):20802085.

11. Tarantini G, Cacciavillani L, Corbetti F, et al. Duration of ischemia is a major determinant of transmurality and severe microvascular obstruction after primary angioplasty: a study performed with contrast-enhanced magnetic resonance. J Am Coll Cardiol. Oct 4 2005;46(7):1229-1235.

12. Comte $A$, Kastler $B$, Laborie $L$, et al. Using a contrast-enhanced imaging sequence at 3-minute delay in 3-T magnetic resonance imaging for acute infarct evaluation. Invest Radiol. Sep 2008;43(9):669675.

13. Nijveldt R, Hofman MB, Hirsch A, et al. Assessment of microvascular obstruction and prediction of short-term remodeling after acute myocardial infarction: cardiac MR imaging study. Radiology. Feb 2009;250(2):363-370.

14. Thygesen K, Alpert JS, White HD, et al. Universal definition of myocardial infarction. Circulation. Nov 27 2007;116(22):2634-2653.

15. Haacke EM BR, Thompson MR. Magnetic Resonance Imaging, Physical Principles and Sequence Design. Vol chapter 8. New York: John Wiley \& Sons; 1999:133-134.

16. Karlsen OT, Verhagen R, Bovee WM. Parameter estimation from Rician-distributed data sets using a maximum likelihood estimator: application to T1 and perfusion measurements. Magn Reson Med. Mar 1999;41(3):614-623.

17. Rochitte CE, Lima JA, Bluemke DA, et al. Magnitude and time course of microvascular obstruction and tissue injury after acute myocardial infarction. Circulation. Sep 8 1998;98(10):1006-1014.

18. Yan AT, Gibson CM, Larose E, et al. Characterization of microvascular dysfunction after acute myocardial infarction by cardiovascular magnetic resonance first-pass perfusion and late gadolinium enhancement imaging. J Cardiovasc Magn Reson. 2006;8(6):831-837. 
19. Gerber BL, Garot J, Bluemke DA, et al. Accuracy of contrast-enhanced magnetic resonance imaging in predicting improvement of regional myocardial function in patients after acute myocardial infarction. Circulation. Aug 27 2002;106(9):1083-1089.

20. Kramer CM, Rogers WJ, Jr., Mankad S, et al. Contractile reserve and contrast uptake pattern by magnetic resonance imaging and functional recovery after reperfused myocardial infarction. J Am Coll Cardiol. Nov 15 2000;36(6):1835-1840.

21. Raff GL, O'Neill WW, Gentry RE, et al. Microvascular obstruction and myocardial function after acute myocardial infarction: assessment by using contrast-enhanced cine MR imaging. Radiology. Aug 2006;240(2):529-536.

22. Stork A, Lund GK, Muellerleile K, et al. Characterization of the peri-infarction zone using T2weighted MRI and delayed-enhancement MRI in patients with acute myocardial infarction. Eur Radiol. Oct 2006;16(10):2350-2357.

23. Judd RM, Lugo-Olivieri $\mathrm{CH}$, Arai $\mathrm{M}$, et al. Physiological basis of myocardial contrast enhancement in fast magnetic resonance images of 2-day-old reperfused canine infarcts. Circulation. Oct 1 1995;92(7):1902-1910.

24. Bogaert J, Kalantzi M, Rademakers FE, et al. Determinants and impact of microvascular obstruction in successfully reperfused ST-segment elevation myocardial infarction. Assessment by magnetic resonance imaging. Eur Radiol. Mar 152007.

25. Ambrosio G, Weisman HF, Mannisi JA, et al. Progressive impairment of regional myocardial perfusion after initial restoration of postischemic blood flow. Circulation. Dec 1989;80(6):1846-1861.

26. Kloner RA, Ganote CE, Jennings RB. The "no-reflow" phenomenon after temporary coronary occlusion in the dog. J Clin Invest. Dec 1974;54(6):1496-1508. 


\title{
CHAPTER 4
}

\author{
Clinical implications of microvascular \\ obstruction and intramyocardial \\ hemorrhage in acute myocardial \\ infarction using cardiovascular magnetic \\ resonance imaging
}

Bekkers SCAM, Smulders MW, Lima Passos V, Leiner T, Waltenberger JL, Gorgels APM, Schalla S

European Radiology 2010; 20(11): 2572-2578 


\begin{abstract}

\section{Objectives}

To investigate the clinical implications of microvascular obstruction (MVO) and intramyocardial hemorrhage (IMH) in acute myocardial infarction (AMI).
\end{abstract}

\title{
Methods
}

Ninety patients with a first AMI undergoing primary percutaneous coronary intervention ( $\mathrm{PCl}$ ) were studied. T2-weighted, cine and delayed enhancement cardiovascular magnetic resonance imaging was performed at $5 \pm 2$ and $103 \pm 11$ days. Patients were categorized into three groups based on the presence or absence of MVO and IMH.

\section{Results}

MVO was observed in $54 \%$ and IMH in $43 \%$ of patients, and correlated significantly $(r=0.8, p<0.001)$. Pre- $\mathrm{PCl}$ thrombolysis in myocardial infarction 3 flow was only observed in $\mathrm{MVO}(-) / \mathrm{IMH}(-)$ patients. Infarct size and impairment of systolic function were largest in $\mathrm{MVO}(+) / \mathrm{IMH}(+)$ patients $(\mathrm{n}=39,23 \pm 9 \%$ and $47 \pm 7 \%)$, smallest in $\mathrm{MVO}(-) / \mathrm{IMH}(-)$ patients $(\mathrm{n}=41,8 \pm 8 \%$ and $55 \pm 8 \%)$ and intermediate in $\mathrm{MVO}(+) / \mathrm{IMH}(-)$ patients $(\mathrm{n}=10,16 \pm 7 \%$ and $51 \pm 6 \%, \mathrm{p}<0.001)$. LVEF increased in all three subgroups at follow-up, but remained intermediate in $\mathrm{MVO}(+) / \mathrm{IMH}(-)$ and was lowest in $\mathrm{MVO}(+) / \mathrm{IMH}(+)$ patients. Using random intercept model analysis, only infarct size was an independent predictor for adverse LV remodeling.

\section{Conclusions}

$\mathrm{IMH}$ and $\mathrm{MVO}$ are strongly related. Pre-PCI TIMI 3 flow is less frequently observed in patients with MVO and IMH. Only infarct size was an independent predictor of LV remodeling. 


\section{Introduction}

Coronary occlusion that persists for more than $40 \mathrm{~min}$. leads to irreversible myocardial damage that starts in the endocardium and progresses towards the epicardium as a wavefront phenomenon. ${ }^{1}$ Although timely reperfusion salvages myocardium and reduces mortality, successful restoration of epicardial artery patency after prolonged occlusion does not always lead to adequate reperfusion at the microvascular level. Reperfused acute myocardial infarction (AMI) is characterized by myocyte swelling due to osmotic overload, intracellular calcium overload, myocyte hypercontraction, microvascular obstruction (MVO) and intramyocardial hemorrhage (IMH). 2,3

Both MVO and IMH occur exclusively in myocardium that sustains the most severe ischemia and are therefore confined to the central portions of infarcted myocardium. ${ }^{3}$ As IMH is caused by extravasation of blood into the extravascular space as a consequence of ischemic microvascular damage, it only occurs in reperfused AMI. ${ }^{4,5}$ IMH does not depend on the magnitude of early reflow but, similar to $\mathrm{MVO}$, on the severity of ischemia as reflected by a longer duration of coronary occlusion and low collateral flow. ${ }^{6,7}$

Cardiovascular magnetic resonance imaging (CMR) is currently regarded as the reference standard to assess myocardial infarction and allows the detection of MVO and IMH in vivo, using delayed enhancement (DE) and T2-weighted (T2W) imaging. ${ }^{8-10}$ Previous studies have suggested that MVO might be associated with adverse ventricular remodeling and clinical outcome. ${ }^{9,11-14}$ The clinical significance of IMH is less well defined, and conflicting results have been reported.

In this study, we sought to investigate the clinical implications of both MVO and $\mathrm{IMH}$ with regard to LV remodeling.

\section{Methods}

\section{Study population}

We studied 90 consecutive patients (65 men, age $60 \pm 11$ years) with a first AMI referred for primary percutaneous coronary intervention $(\mathrm{PCI})$ and presentation $<12$ $\mathrm{h}$ after symptom onset. The definition of AMI was based on the recent consensus document including appropriate rise and fall in cardiac biomarkers. ${ }^{17}$ Excluded were patients $<18$ years and those with contraindications for CMR. The institutional review board of our hospital approved the study, and patients were included after written informed consent was obtained.

Before emergency $\mathrm{PCl}$ all patients received $500 \mathrm{mg}$ aspirin, 5,000 $\mathrm{U}$ heparin and $600 \mathrm{mg}$ clopidogrel. The culprit coronary artery was the LAD in 31\%, the RCA in $56 \%$ 
and LCX in $13 \%$ of patients; single vessel disease was present in $52 \%$, two vessel disease in $23 \%$ and three vessel disease in $25 \%$. All patients received standard post$\mathrm{PCl}$ care, including dual antiplatelet therapy for at least 1 month. Thrombosuction, which was not standard treatment at the time of this study, and downstream administration of intravenous abciximab, intracoronary nitroglycerine and adenosine were left to the discretion of the interventional cardiologist.

\section{Cardiovascular magnetic resonance imaging protocol}

CMR was performed at $5 \pm 2$ days and $103 \pm 11$ days after admission. Images were acquired on a 1.5 Tesla MRI system (Intera, Philips Medical Systems, Best, The Netherlands) with a dedicated five-element phased array surface coil. For functional analysis, ECG-gated cine images were obtained in the LV short axis plane covering the entire LV using a segmented balanced steady-state free precession sequence (slice thickness $6 \mathrm{~mm}$, slice gap $4 \mathrm{~mm}$, average repetition time (TR) and echo time (TE) 3.8/1.9 ms, respectively, flip angle $50^{\circ}$, FOV $350 \mathrm{~mm}$, matrix $256 \times 256$, typically 22-25 phases per cardiac cycle). Next, a breath-hold, multislice, black blood T2weighted turbo spin-echo sequence with short inversion time and fat suppression was used (T2 SPIR, slice thickness $8 \mathrm{~mm}$, slice gap $2 \mathrm{~mm}$, TR two R-R intervals and TE $100 \mathrm{~ms}$, FOV $350 \mathrm{~mm}$, matrix 236 x 186). DE CMR was performed $10 \mathrm{~min}$. after an intravenous bolus of $0.2 \mathrm{mmol} / \mathrm{kg}$ body weight gadolinium-diethylenetriaminepentaacetic acid (Magnevist ${ }^{\circledR}$, Bayer Schering Pharma, Berlin, Germany) using a breathhold three-dimensional inversion-recovery gradient-echo sequence (acquired slice thickness $12 \mathrm{~mm}$, reconstructed slice thickness $6 \mathrm{~mm}$, average TR/TE 3.9/2.4 ms, multi-shot (50 profiles/shot) segmented partial echo readout every heart beat (TFE), flip angle $15^{\circ}$, FOV $400 \mathrm{~mm}$, matrix $256 \times 256$, acquired and reconstructed pixel size $1.56 \times 1.56 \mathrm{~mm}$ ). The inversion time that optimally suppressed signal of non-infarcted myocardium (typical range 200-280 ms) was determined with a preceding Look-Locker sequence.

\section{CMR image analysis}

The CMR images were analyzed independently by two observers blinded to clinical data, using commercially available software (CAAS MRV 3.0, Pie Medical Imaging, Maastricht, The Netherlands). The interobserver agreement was excellent ( $\mathrm{k}$ value 0.9). Discrepancies were resolved in consensus. Endocardial and epicardial borders were manually traced, excluding the papillary muscles, in the end-diastolic and endsystolic short-axis phases to determine left ventricular end-diastolic volume (LVEDV), end-systolic volume (LVESV), stroke volume (LVSV), ejection fraction (LVEF) and end-diastolic mass (LV mass). These parameters were indexed for body surface area. 
Likewise, endocardial and epicardial contours were manually traced on the DE and $\mathrm{T} 2 \mathrm{~W}$ images, which were viewed as separate sets. The T2W images of the first CMR examination were used to determine the presence or absence of $\mathrm{IMH}$, defined as a central hypoenhanced area within the hyperintense edematous area, indicative of the area at risk (AAR). The AAR was quantified by semiautomatic detection using a signal intensity (SI) threshold of $>2$ SD above a remote non-infarcted region and expressed as a percentage of LV mass. Subendocardial 'slow flow' artifacts were carefully excluded, and areas of IMH were included in the AAR analysis by manual adjustment of contours. IMH was manually traced and expressed as a percentage of LV mass.

Infarct size (IS) was quantified on the DE images of the first CMR using an SI threshold of $>5$ SD above a remote non-infarcted reference region, including areas of MVO (central hypoenhancement within hyperenhanced area) and expressed as a percentage of LV mass. MVO was quantified by manually tracing the central hypoenhanced area and expressed as a percentage of LV mass.

\section{Statistics}

Summary statistics of continuous data with symmetric distribution are expressed as mean \pm standard deviation (SD), otherwise as median with interquartile range (IQR). Categorical data are expressed as frequencies with percentages. Univariate group comparisons were conducted with the one-way ANOVA or the non-parametric Kruskal-Wallis test for continuous variables. For categorical variables, chi-square or Fisher's exact tests were applied. Adjustment for multiple testing was conducted via the false discovery rate (FDR). Detected differences were considered significant when the corrected FDR $p$-value was $<0.05$. A multiple OLS linear regression model was fitted to quantify the association between $\mathrm{IMH}$ and $\mathrm{MVO}$ for the $\mathrm{MVO}(+) / \mathrm{IMH}(+)$ group, adjusting for other clinical variables. To stabilize residual variance, IMH and MVO values, expressed as a proportion of the LV mass, were logarithmically transformed (In). The final model was used to predict IMH size for the remaining $10 \mathrm{MVO}(+) / \mathrm{IMH}(-)$ patients. The estimated $\mathrm{IMH}$ values could provide some indication of the possible underlying reasons for the absence of IMH in these patients. A random intercept model analysis was conducted to determine independent predictors of LV remodeling. Statistical analysis was performed with SPSS software (version 17.0 for Windows; SPSS Inc., Chicago, IL). 

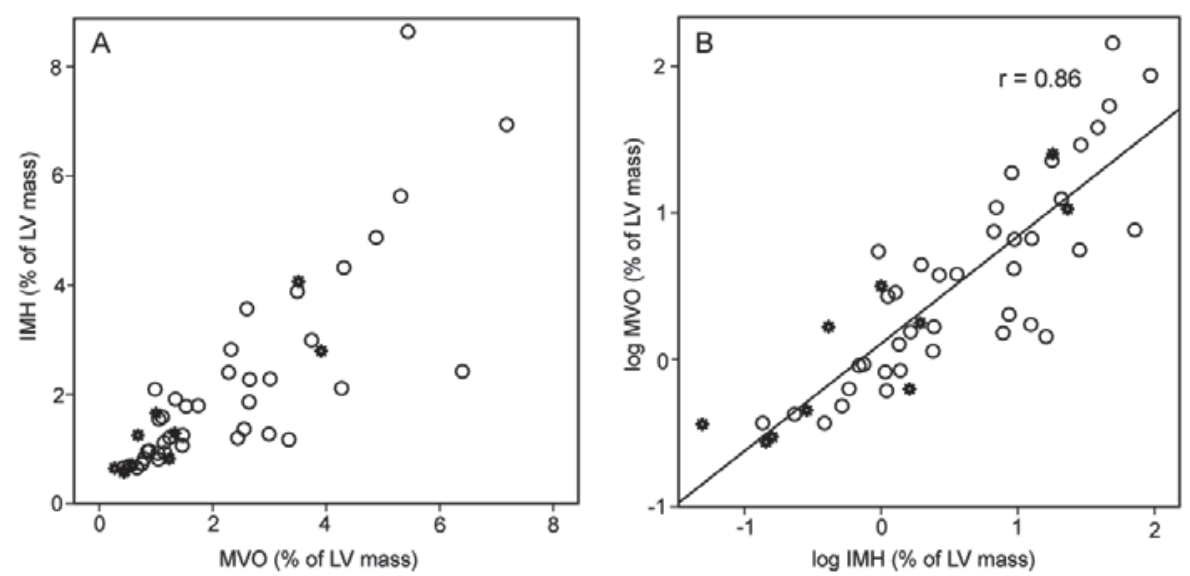

Figure 1. Correlation between IMH and MVO. (A) Original scale values. (B) Log transformed values. A good linear correlation is seen between MVO and IMH $(r=0.86, p<0.001)$. Open circles indicate observed values and stars indicate predicted values in the $\mathrm{MVO}(+) / \mathrm{IMH}(-)$ group. Note that most of the predicted values are within the lower range of observed values.

\section{Results}

\section{IMH and MVO}

Signal intensity on the T2W and LGE images within the territory of the IRA was increased in all patients. The AAR and IS were $26 \pm 12 \%$ and $15 \pm 11 \%$ of LV mass, respectively. MVO was observed in 49 (54\%) and IMH in 39 patients (43\%), and both were always located subendocardially within the infarct core. IMH was only observed in patients with MVO. A significant correlation was found between MVO and IMH extent ( $r=0.8, p<0.001$, Figure 1$)$, but absolute MVO extent was larger than that of IMH [3.1 ml (IQR 1.6-5.3) vs. $2.2 \mathrm{ml}$ (IQR 1.6-3.5), $p=0.04]$.

\section{Subgroups}

Based on the presence or absence of $\mathrm{MVO}$ and $\mathrm{IMH}$, patients were classified into three groups: 41 patients with $\mathrm{MVO}(-) / \mathrm{IMH}(-), 10$ with $\mathrm{MVO}(+) / \mathrm{IMH}(-)$ and 39 with $\mathrm{MVO}(+) / \mathrm{IMH}(+)$ (Figure 2). For most of the baseline characteristics, there were no significant differences between groups (Table 1), except for pre-PCI TIMI 3 flow, which was only observed in the $\mathrm{MVO}(-) / \mathrm{IMH}(-)$ group. There was no difference in the use of glycoprotein Ilbllla inhibitor between groups.

The AAR and IS were largest in the $\mathrm{MVO}(+) / \mathrm{IMH}(+)$ group, intermediate in the $\mathrm{MVO}(+) / \mathrm{IMH}(-)$ group and smallest in the $\mathrm{MVO}(-) / \mathrm{IMH}(-)$ group (Table 1$)$. 
Table 1. Baseline characteristics.

\begin{tabular}{|c|c|c|c|c|}
\hline & \multicolumn{3}{|c|}{ Group } & \multirow[b]{2}{*}{ FDR } \\
\hline & MVO (-)/IMH(-) & $\mathrm{MVO}(+) / \mathrm{IMH}(-)$ & $\mathrm{MVO}(+) / \mathrm{IMH}(+)$ & \\
\hline Age (years) & $61 \pm 9$ & $59 \pm 15$ & $59 \pm 12$ & 0.64 \\
\hline Male (\%) & $27(66)$ & $6(60)$ & $32(82)$ & 0.29 \\
\hline DM (\%) & $4(10)$ & $1(10)$ & $1(3)$ & 0.49 \\
\hline Smoking (\%) & $33(80)$ & $9(90)$ & $36(92)$ & 0.38 \\
\hline Hypertension (\%) & $20(49)$ & $3(30)$ & $12(31)$ & 0.35 \\
\hline Hypercholesterolemia (\%) & $11(27)$ & $3(30)$ & $11(28)$ & 0.95 \\
\hline Positive family history (\%) & $16(39)$ & $3(30)$ & $22(56)$ & 0.29 \\
\hline Anterior location (\%) & $10(24)$ & $2(20)$ & $16(41)$ & 0.30 \\
\hline Previous angina (\%) & $13(32)$ & $3(30)$ & $21(54)$ & 0.20 \\
\hline Gllbllla inhibitor (\%) & $17(41)$ & $5(50)$ & $23(59)$ & 0.38 \\
\hline \multicolumn{5}{|l|}{ TIMI 3} \\
\hline Pre-PCI (\%) & $10(24)$ & $0(0)$ & $0(0)$ & 0.004 \\
\hline Rentrop $\geq 2(\%)$ & $9(22)$ & $5(50)$ & $7(18)$ & 0.20 \\
\hline Thrombosuction (\%) & $9(22)$ & $2(20)$ & $11(28)$ & 0.89 \\
\hline Time to $\mathrm{PCl}$ (min.) & $217(165-304)$ & $177(148-248)$ & $201(160-291)$ & 0.65 \\
\hline AAR (\%) & $19 \pm 12^{*^{+}+}$ & $27 \pm 8^{*}$ & $33 \pm 9^{+}$ & $<0.001$ \\
\hline IS (\%) & $8 \pm 8^{*+}$ & $16 \pm 7^{* \prime}$ & $23 \pm 9^{\dagger, \neq}$ & $<0.001$ \\
\hline Myocardial salvage (\%) & $54 \pm 32^{*}$ & $40 \pm 25$ & $28 \pm 24^{*}$ & 0.004 \\
\hline MVO (\% of LV mass) & 0 & $0.8(0.4-1.9)$ & $1.7(1.0-3.3)$ & 0.07 \\
\hline IMH (\% of LV mass) & 0 & 0 & $1.6(1.0-2.4)$ & \\
\hline
\end{tabular}

Values are presented as mean \pm SD or median and IQR; Abbreviations: $A A R=$ area at risk; $D M=$ diabetes mellitus; IS=infarct size at baseline; IHM=intramyocardial hemorrhage; $M V O=$ microvascular obstruction; $\mathrm{PCl}=$ percutaneous coronary intervention. Superscripts $\left(*^{+},{ }^{\ddagger}\right)$ indicate significant post-hoc, pairwise group comparison.

Myocardial salvage, defined as the difference between AAR and IS, was lowest in the $\mathrm{MVO}(+) / \mathrm{IMH}(+)$ group, intermediate in the $\mathrm{MVO}(+) / \mathrm{IMH}(-)$ group and highest in the $\mathrm{MVO}(-) / \mathrm{IMH}(-)$ group $(28 \pm 24 \%, 40 \pm 25 \%$ and $54 \pm 32 \%$, respectively, $p<0.001$, Table 1 and Figure 3).

The MVO extent tended to be larger in the $\mathrm{MVO}(+) / \mathrm{IMH}(+)$ patients compared with the $\mathrm{MVO}(+) / \mathrm{IMH}(-)$ patients (1.7\% (IQR 1.0-3.3\%) vs. $0.8 \%$ (IQR $0.4-1.9 \%$ ) of LV mass, respectively, $p=0.07)$. 

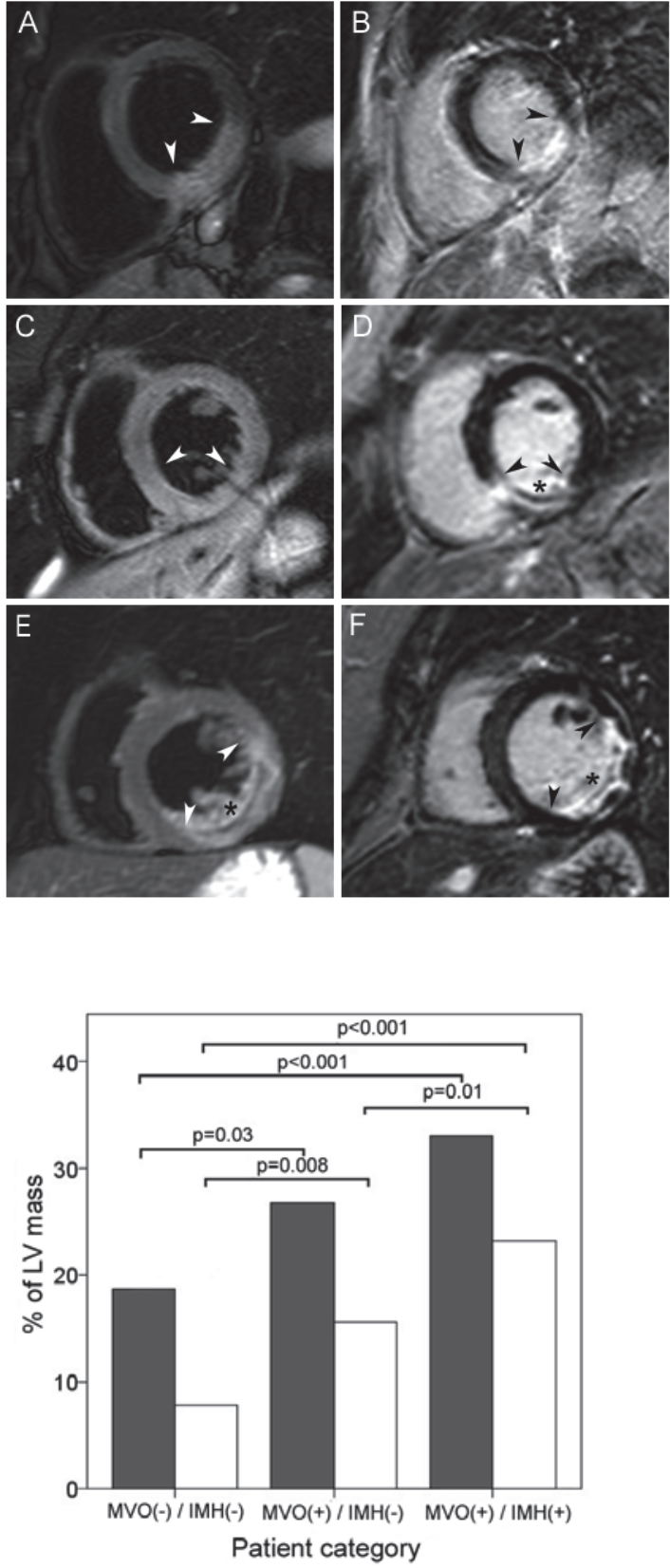

Figure 2. Magnetic resonance image examples from each patient group. Top row (A, B): $\mathrm{MVO}(-) / \mathrm{IMH}(-)$ patient; middle row (C, D): $\mathrm{MVO}(+) / \mathrm{IMH}(-)$ patient; bottom row (E, F): $\mathrm{MVO}(+) /$ $(\mathrm{IMH}(+)$ patient. T2-weighted images are shown on the left $(A, C, E)$ and corresponding delayed enhancement images on the right (B, D, F). Edema and infarct border zones are indicated by arrowheads and IMH and MVO by asterisks.
Figure 3. Area at risk (AAR) and infarct size (IS). AAR (grey bars) and IS (white bars) represented as percentages of LV mass for each patient group. The $\mathrm{MVO}(+) / \mathrm{IMH}(-)$ group represents an intermediate group. Myocardial salvage is the difference between AAR and IS. 


\section{Predicted IMH size in patients with MVO but without IMH}

The IMH in the $\mathrm{MVO}(+) / \mathrm{IMH}(-)$ patients could have been missed as a result of the limits of spatial resolution of T2W-CMR. To investigate this, predicted IMH size values for $\mathrm{MVO}(+) / \mathrm{IMH}(-)$ were calculated based on the following model derived from the $\mathrm{MVO}(+) / \mathrm{IMH}(+)$ group data:

$$
(\text { In_IMH }=-0.348+0.718 \times \text { In_MVO }+0.454 \times \text { sex }+0.395 \times \text { smoke }) .
$$

All predictors in the above model were statistically significant. Observed and predicted IMH values are displayed in Figure $1 \mathrm{~A}$ and $1 \mathrm{~B}$. Predicted values are marked as stars. In either case it is evident that only two patients had sufficiently small predicted IMH outside the observed value range in the other patients. Theoretically, these two values could have been missed by T2W-CMR. Conversely, several of the predicted values were still of sufficient size, at least comparable to that of the observed counterparts, and as such they could have been detected by CMR.

Table 2. Functional parameters at baseline and follow up.

\begin{tabular}{|c|c|c|c|c|}
\hline & \multicolumn{3}{|c|}{ Group } & \multirow[b]{2}{*}{ FDR } \\
\hline & MVO (-)/IMH(-) & MVO(+)/IMH(-) & MVO (+)/IMH(+) & \\
\hline \multicolumn{5}{|l|}{ LVEDVi $\left(\mathrm{mL} / \mathrm{m}^{2}\right)$} \\
\hline Baseline & $81 \pm 14^{*}$ & $86 \pm 15$ & $88 \pm 17^{*}$ & 0.24 \\
\hline Follow-up & $78 \pm 18^{\dagger}$ & $76 \pm 12$ & $88 \pm 20^{\dagger}$ & 0.13 \\
\hline \multicolumn{5}{|l|}{ LVESVi $\left(\mathrm{mL} / \mathrm{m}^{2}\right)$} \\
\hline Baseline & $38 \pm 11^{*}$ & $43 \pm 11$ & $47 \pm 13^{*}$ & 0.01 \\
\hline Follow-up & $34 \pm 12^{+}$ & $34 \pm 6$ & $45 \pm 15^{+}$ & 0.01 \\
\hline \multicolumn{5}{|l|}{ LVEF (\%) } \\
\hline Baseline & $55 \pm 8^{*}$ & $51 \pm 6$ & $47 \pm 7^{*}$ & $<0.001$ \\
\hline Follow-up & $57 \pm 7^{+}$ & $55 \pm 3$ & $50 \pm 7^{\dagger}$ & $<0.001$ \\
\hline
\end{tabular}

Values are presented as mean \pm SD; Abbreviations: IMH=intramyocardial hemorrhage; LVEDV $_{\mathrm{i}}=$ left ventricular end diastolic volume index; LVESV $_{\mathrm{i}}=$ left ventricular end-systolic volume index; LVEF=left ventricular ejection fraction; $\mathrm{MVO}=$ microvascular obstruction. Superscripts $\left({ }^{*}{ }^{+}\right)$indicate significant post-hoc, pairwise group comparison. 


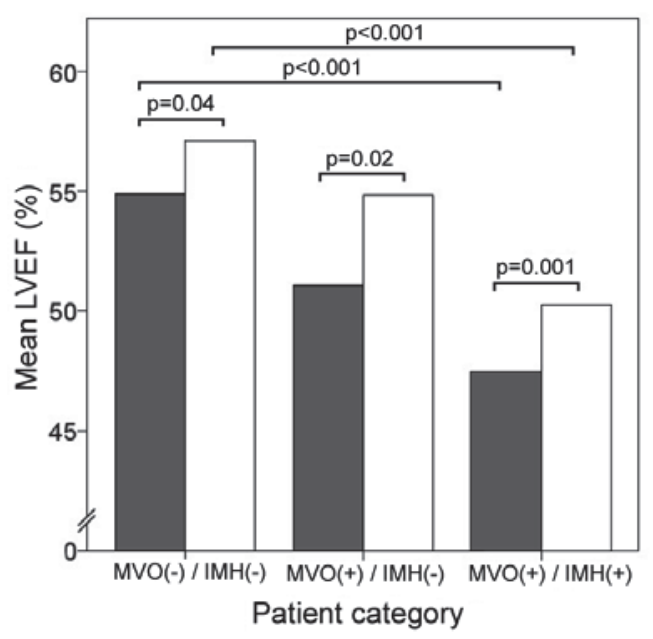

Figure 4. Left ventricular ejection fraction (LVEF) at baseline and follow-up. LVEF significantly increased in all three groups from baseline (grey bars) to follow-up CMR (white bars), but remained intermediate in the $\mathrm{MVO}(+) / \mathrm{IMH}(-)$ group

\section{Functional parameters and LV remodeling}

The LVEDV $V_{i}$ and LVESV ${ }_{i}$ decreased from baseline to follow-up and were highest in the $\mathrm{MVO}(+) / \mathrm{MVO}(+)$ group, intermediate in the $\mathrm{MVO}(+) / \mathrm{IMH}(-)$ group and lowest in the $\mathrm{MVO}(-) / \mathrm{IMH}(-)$ group at both time points (Table 2 ). Likewise, LVEF improved in all three groups, but remained lowest in the $\mathrm{MVO}(+) / \mathrm{MVO}(+)$ group, intermediate in the $\mathrm{MVO}(+) / \mathrm{IMH}(-)$ group and highest in the $\mathrm{MVO}(-) / \mathrm{IMH}(-)$ group in the univariate comparisons (Figure 4).

Table 3 displays the fixed effect parameter estimates of the random intercept model. Subjects were taken as random effect, whereas time and $\mathrm{MVO} / \mathrm{IMH}$ groups were taken as fixed effect factors adjusted for IS. LVEF significantly increased over time (for all groups), showing a significant negative association with IS. Note that the presence of IMH or MVO had no significant independent predictive value for LVEF once adjusted for IS.

Table 3. Fixed effect parameter estimates of the random intercept model. Outcome variable: LVEF.

\begin{tabular}{lll}
\hline & $\beta(S E)$ & $p$-value \\
\hline Intercept & $55.651(2.086)$ & $<0.001$ \\
IS & $-0.452(0,071)$ & $<0.001$ \\
Time & $2.660(0.623)$ & $<0.001$ \\
$\mathrm{MVO}(-) / \mathrm{IMH}(-)$ & $-0.023(1.648)$ & 0.989 \\
$\mathrm{MVO}(+) / \mathrm{IMH}(-)$ & $0.004(2.059)$ & 0.998 \\
$\mathrm{MVO}(+) / \mathrm{IMH}(+)$ & reference & \\
\hline
\end{tabular}

Abbreviations: IS=infarct size; $\mathrm{MVO}=$ microvascular obstruction. Error and random intercept variances were 15.762 and 20.571, respectively $(p<0.001)$. 


\section{Discussion}

The findings of our study can be summarized as follows: (1) MVO and IMH are frequently observed in reperfused AMI using T2W and LGE CMR, (2) IMH and MVO are strongly related phenomena, (3) patients with MVO and IMH have pre-PCI TIMI 3 flow less often, and (4) infarct size, once adjusted for MVO and IMH presence, remained the sole independent predictor of LV remodeling.

T2W-CMR is capable of detecting myocardial edema as regions of increased signal intensity due to prolongation of $\mathrm{T} 2$ relaxation time as a consequence of increased tissue free water content. ${ }^{18} \mathrm{IMH}$ is thought to be caused by leakage of blood out of severely injured microvasculature into the interstitium that can be depicted as a central area of low signal intensity (SI) within the edematous area on T2W-CMR. This low SI is explained by local field heterogeneities due to the paramagnetic effects of blood degradation products such as deoxyhaemoglobin, which shorten the $\mathrm{T} 2$ relaxation time. ${ }^{10}$ In two case reports, Basso et al. demonstrated a good correlation among the location, spatial extent, and shape of IMH on CMR and histopathology. ${ }^{8}$ Because of its excellent spatial resolution and high contrast, LGE CMR accurately assesses the extent of infarction and MVO. ${ }^{19}$ Combined with T2W$\mathrm{CMR}$, it is now possible to gain novel insights into the pathophysiology of IMH and MVO in vivo and evaluate their clinical significance.

Previous studies have shown that MVO infarcts show a lack of regional and global functional recovery compared with non-MVO infarcts; however, it remains unclear whether MVO provides independent prognostic information. ${ }^{11,12}$ Despite the strong relation of MVO with IS, some studies indicated that MVO is an important determinant of LV recovery and prognosis, even after controlling for IS, whereas others have reported that MVO loses its predictive power after adjusting for IS. 13, 14, 20, 21 Persistence of MVO, but not MVO that disappeared at 1 week after infarction, was associated with attenuated infarct healing and subsequent adverse remodeling, indicating that the timing of MVO imaging is important. ${ }^{22}$ In that study and similar to our findings, IS remained the major determinant of LV remodeling in a multivariate model.

Our clinical observation that MVO and IMH occur in the central portions of the infarct and not in patients with pre-PCI TIMI 3 flow is in line with experimental studies showing the association of IMH with markedly depressed flow before reperfusion and severe ischemia. ${ }^{5}$ Our finding that IMH was also observed in $10 \%$ of patients with post-PCI TIMI flow $<3$ supports experimental observations that IMH does not depend on the magnitude of early reflow. ${ }^{6,23}$ Conflicting results on the clinical significance of IMH have been reported. Our data are in contrast to those of Ganame et al., who found that LVEF did not improve in patients with hemorrhagic infarcts as opposed to patients with non-hemorrhagic infarcts. ${ }^{16} \mathrm{IMH}$ and infarct size were the strongest independent predictors of adverse LV remodeling in that 
study. Our results are in accordance with those from Beek et al., who showed that IMH did not have prognostic significance beyond the presence of MVO. ${ }^{15}$

The underlying pathophysiological mechanisms of MVO and IMH are complex and remain only partially understood. Irreversible ischemic myocardial cell injury progresses as a 'wave front' from the endocardium to the epicardium, with endothelial cell injury occurring after myocardial cell injury. ${ }^{1,3}$ After varying periods of ischemia, sequential changes of the microvasculature occur. Endothelial protrusion by cell swelling together with capillary plugging by neutrophils, red blood cells and platelets and swelling of surrounding myocytes compressing capillaries may all cause MVO. As ischemic injury progresses, endothelial cell death causes loss of capillary integrity with resultant IMH in cases of myocardial reperfusion. Our data show that MVO and IMH are strongly related phenomena and suggest that IMH is a sign of severely injured microvasculature that easily leaks blood into the interstitial space. Furthermore, our results suggest that microvascular injury is predominantly a reflection of extensive myocardial necrosis rather than a separate entity identifying patients at higher risk.

Despite having MVO, IMH was absent in ten patients in our study. We postulate that, for some patients in the $\mathrm{MVO}(+) / \mathrm{IMH}(-)$ group, the ischemic threshold for $\mathrm{IMH}$ may not have been reached yet. This could explain the discrepancy between the predicted IMH values and the negative findings at CMR. Lending support to this concept was the finding that all measured parameters, including MVO size, were in between the average values of the two other groups. It is therefore likely that the groups reflect a gradation of the severity of ischemic damage.

\section{Conclusions}

Microvascular obstruction and intramyocardial hemorrhage are frequently observed in reperfused $\mathrm{AMI}$ using $\mathrm{T} 2 \mathrm{~W}$ and $\mathrm{LGE}-\mathrm{CMR}$, and are strongly related phenomena. Patients with MVO and IMH have pre-PCI TIMI 3 flow less often, suggesting more severe ischemia before reperfusion. Only infarct size, but not the presence of IMH or MVO, was an independent predictor for LVEF. This suggests that MVO and IMH are not just separate entities, but rather ischemia-dependent consequences of severely injured microvasculature in larger infarcts. 


\section{References}

1. Reimer KA, Lowe JE, Rasmussen MM, et al. The wavefront phenomenon of ischemic cell death. 1. Myocardial infarct size vs duration of coronary occlusion in dogs. Circulation. Nov 1977;56(5):786794.

2. Bresnahan GF, Roberts R, Shell WE, et al. Deleterious effects due to hemorrhage after myocardial reperfusion. Am J Cardiol. Jan 1974;33(1):82-86.

3. Kloner RA, Ganote CE, Whalen DA, Jr., et al. Effect of a transient period of ischemia on myocardial cells. II. Fine structure during the first few minutes of reflow. Am J Pathol. Mar 1974;74(3):399-422.

4. Basso C, Thiene G. The pathophysiology of myocardial reperfusion: a pathologist's perspective. Heart. Nov 2006;92(11):1559-1562.

5. Fishbein MC, J YR, Lando $U$, et al. The relationship of vascular injury and myocardial hemorrhage to necrosis after reperfusion. Circulation. Dec 1980;62(6):1274-1279.

6. Garcia-Dorado D, Theroux P, Solares J, et al. Determinants of hemorrhagic infarcts. Histologic observations from experiments involving coronary occlusion, coronary reperfusion, and reocclusion. Am $J$ Pathol. Aug 1990;137(2):301-311.

7. Higginson LA, White F, Heggtveit HA, et al. Determinants of myocardial hemorrhage after coronary reperfusion in the anesthetized dog. Circulation. Jan 1982;65(1):62-69.

8. Basso C, Corbetti F, Silva C, et al. Morphologic validation of reperfused hemorrhagic myocardial infarction by cardiovascular magnetic resonance. Am J Cardiol. Oct 15 2007;100(8):1322-1327.

9. Hombach V, Grebe O, Merkle N, et al. Sequelae of acute myocardial infarction regarding cardiac structure and function and their prognostic significance as assessed by magnetic resonance imaging. Eur Heart J. Mar 2005;26(6):549-557.

10. Lotan CS, Bouchard A, Cranney GB, et al. Assessment of postreperfusion myocardial hemorrhage using proton NMR imaging at 1.5 T. Circulation. Sep 1992;86(3):1018-1025.

11. Baks T, van Geuns RJ, Biagini E, et al. Effects of primary angioplasty for acute myocardial infarction on early and late infarct size and left ventricular wall characteristics. J Am Coll Cardiol. Jan 3 2006;47(1):40-44.

12. Bogaert J, Kalantzi M, Rademakers FE, et al. Determinants and impact of microvascular obstruction in successfully reperfused ST-segment elevation myocardial infarction. Assessment by magnetic resonance imaging. Eur Radiol. Oct 2007;17(10):2572-2580.

13. Nijveldt R, Beek AM, Hirsch A, et al. Functional recovery after acute myocardial infarction: comparison between angiography, electrocardiography, and cardiovascular magnetic resonance measures of microvascular injury. J Am Coll Cardiol. Jul 15 2008;52(3):181-189.

14. Wu KC, Zerhouni EA, Judd RM, et al. Prognostic significance of microvascular obstruction by magnetic resonance imaging in patients with acute myocardial infarction. Circulation. Mar 3 1998;97(8):765-772.

15. Beek AM, Nijveldt R, van Rossum AC. Intramyocardial hemorrhage and microvascular obstruction after primary percutaneous coronary intervention. Int J Cardiovasc Imaging. Jan 2010;26(1):49-55.

16. Ganame J, Messalli G, Dymarkowski S, et al. Impact of myocardial haemorrhage on left ventricular function and remodelling in patients with reperfused acute myocardial infarction. Eur Heart J. Jun 2009;30(12):1440-1449.

17. Thygesen K, Alpert JS, White HD, et al. Universal definition of myocardial infarction. Circulation. Nov 27 2007;116(22):2634-2653.

18. Simonetti OP, Finn JP, White RD, et al. "Black blood" T2-weighted inversion-recovery MR imaging of the heart. Radiology. Apr 1996;199(1):49-57.

19. Kim RJ, Fieno DS, Parrish TB, et al. Relationship of MRI delayed contrast enhancement to irreversible injury, infarct age, and contractile function. Circulation. Nov 9 1999;100(19):1992-2002.

20. Shapiro MD, Nieman K, Nasir K, et al. Utility of cardiovascular magnetic resonance to predict left ventricular recovery after primary percutaneous coronary intervention for patients presenting with acute ST-segment elevation myocardial infarction. Am J Cardiol. Jul 15 2007;100(2):211-216. 
21. Tarantini G, Razzolini R, Cacciavillani L, et al. Influence of transmurality, infarct size, and severe microvascular obstruction on left ventricular remodeling and function after primary coronary angioplasty. Am J Cardiol. Oct 15 2006;98(8):1033-1040.

22. Orn S, Manhenke C, Greve OJ, et al. Microvascular obstruction is a major determinant of infarct healing and subsequent left ventricular remodelling following primary percutaneous coronary intervention. Eur Heart J. Aug 2009;30(16):1978-1985.

23. Roberts CS, Schoen FJ, Kloner RA. Effect of coronary reperfusion on myocardial hemorrhage and infarct healing. Am J Cardiol. Sep 1 1983;52(5):610-614. 


\section{CHAPTER 5}

\section{ST segment recovery in relation to underlying infarct characteristics and adverse remodeling as assessed by cardiovascular magnetic resonance} imaging 


\begin{abstract}
Background

The relation of early ST segment recovery (STR) with underlying infarct characteristics and adverse remodeling in ST elevation myocardial infarction (STEMI) is incompletely known.
\end{abstract}

\title{
Methods
}

In 79 first STEMI patients, eight different ST segment recovery (STR) variables were measured 30 and 60 minutes after percutaneous coronary intervention. Cardiovascular magnetic resonance imaging (CMR) was performed $5 \pm 2$ and $104 \pm 11$ days after admission. Using $K$-means cluster analysis, three CMR risk groups for adverse remodeling were identified (low, $n=16$; intermediate, $n=28$; high risk, $n=35$ ) based on combinations of infarct size (IS), transmurality and microvascular obstruction (MVO). Stepwise discriminant analysis was used to determine which STR variable best discriminated between groups.

\section{Results}

Clustering based on MVO and IS allowed a better separation between groups than clustering based on MVO, IS and transmurality, in relation to adverse remodeling. Baseline LVEF improved in all groups, but remained lowest in the high risk group (41 $\pm 7 \%$ and $44 \pm 6 \%$ ), as compared to the intermediate ( $51 \pm 5 \%$ and $56 \pm 5 \%$ ) and low risk group (56 $\pm 7 \%$ and $58 \pm 5 \%$ ). IS was significantly different among all groups (34 $\pm 5 \%, 19 \pm 4 \%$ and $6 \pm 4 \%, p<0.001)$ and mainly determined the effect on adverse remodeling. Of all STR variables, worst lead residual ST deviation (STD) 30 minutes after reperfusion accurately discriminated between the high and combined low/intermediate risk groups.

\section{Conclusion}

Worst lead residual STD 30 minutes after reperfusion allows accurate identification of patients at high risk for adverse remodeling after 3 months, which was mainly related to IS rather than transmurality or MVO. 


\section{Introduction}

Timely reperfusion in ST elevation myocardial infarction (STEMI) increases myocardial salvage limits infarct size (IS) and improves clinical outcome. ${ }^{1}$ A substantial number of patients fails to have adequate microvascular reperfusion despite successful restoration of epicardial artery patency. ${ }^{2,3}$

Studies comparing angiographic measures of reperfusion with ST segment recovery (STR) early after treatment (early STR) have shown a significant correlation between greater STR and higher rates of TIMI grade 3 flow. ${ }^{4}$ Incomplete STR in patients with TIMI grade 3 flow is widely accepted as an indicator of impaired tissue reperfusion, but only few studies have directly related STR to microvascular obstruction (MVO). ${ }^{5-8}$ It appears that incomplete STR is also related to other infarct characteristics such as decreased myocardial salvage, increased IS, decreased left ventricular ejection fraction (LVEF) and consequently, adverse clinical outcome. ${ }^{9-11}$

Functional recovery after STEMI is related to IS, infarct transmurality and MVO. Studies have reported inconsistent results, with some favoring $\mathrm{IS}^{12}$, and others $\mathrm{MVO}^{7}$ or transmurality ${ }^{13}$, as independent predictors for adverse ventricular remodeling and outcome. Moreover, these infarct characteristics appear to be strongly interrelated, hindering the elucidation of the relationship with STR. Thus, the exact relationship of STR with these underlying infarct characteristics and adverse remodeling remains to be established.

Cardiovascular magnetic resonance imaging (CMR) allows an accurate and integrative assessment of IS, MVO, transmurality and LVEF ${ }^{13-15}$ and was used to investigate the relationship of STR with underlying infarct characteristics and adverse remodeling.

\section{Methods}

The study was approved by the ethics committee of our institution and patients were included after written informed consent was obtained.

\section{Study population}

We studied 86 consecutive patients with a first STEMI, who were referred to our institution between September 2006 and March 2008 for primary percutaneous coronary intervention $(\mathrm{PCl})$. Patients were included when they had typical and prolonged ( $>30$ minutes) chest pain, ST elevation at the J-point $\geq 0.2 \mathrm{mV}$ in leads $V_{2}-V_{3}$ and/or $\geq 0.1 \mathrm{mV}$ in other leads in $\geq 2$ contiguous leads and an appropriate rise and fall in cardiac biomarkers. All patients received aspirin, heparin and clopidogrel before $\mathrm{PCl}$ and stenting. Thrombosuction and downstream administration of intra- 
venous abciximab, intracoronary nitroglycerine or adenosine were left at the discretion of the interventional cardiologist. Patients with left bundle branch block and (relative) contraindications for CMR were excluded. The ECG recordings and CMR images were non-diagnostic due to artifacts in 4 and 3 patients, respectively, leaving 79 patients for the final analysis.

Cardiac biomarkers were sampled on admission and at 6-hour intervals until 72 hours after admission. Creatine kinase area-under-the-curve (CK AUC) was derived and expressed as $1,000 \mathrm{~h} \cdot$ units $^{-1}$.

\section{ECG analysis}

Immediately upon admission and prior to $\mathrm{PCl}$, each patient received a continuous 24-h digital 12-lead Holter recorder (NEMON 180+, NorthEast Monitoring, Inc., Maynard, MA). The system acquires and stores a digital and complete Mason-Likar 12-lead ECG every minute. ST segments were measured manually, 60 ms after the Jpoint with the TP-segment as isoelectric line at baseline, 30 and 60 minutes after balloon inflation, using designated analysis software (Holter 5 LX Analysis version 5.2, NorthEast Monitoring). ST elevation in leads $\mathrm{V}_{1}$ to $\mathrm{V}_{6}$, and /or I and aVL was defined as anterior, ST elevation in leads II, III, aVF and/or ST depression in leads $\mathrm{V}_{1}$ to $\mathrm{V}_{4}$ as non-anterior STEMI. In all patients, single lead peak ST deviation (STD) and sum peak STD (sum of ST segment elevation plus ST segment depression) were measured.

Then, the following STR variables were calculated: 1) $\Sigma$ STR (30): percent reduction in total STD from baseline to 30 minutes post-PCl; 2) $\Sigma$ STR (60): percent reduction in total STD from baseline to 60 minutes post-PCI; 3) STR (30): percent reduction in single lead peak STD from baseline to 30 minutes post-PCI; 4) STR(60): percent reduction in single lead peak STD from baseline to 60 minutes post-PCl; 5) $\Sigma$ STD (30): sum of residual STD 30 minutes post-PCl; 6) $\Sigma$ STD (60): sum of residual STD 60 minutes post-PCl; 7) STD (30): worst lead residual STD 30 minutes post-PCl; 8) STD (60): worst lead residual STD 60 minutes post-PCI. Single lead STR was calculated consistently in the lead with maximum baseline STD, worst lead residual STD was measured in the lead with maximum STD post-PCl, regardless if baseline peak STD was in a different lead. ECG's were independently analyzed by two observers, blinded to the clinical and CMR data. The interclass correlation coefficient, as a measure of agreement, was 0.95 with $95 \% \mathrm{Cl}(0.92,0.97)$. Discrepancies were resolved by consensus repeat analysis.

\section{Cardiovascular magnetic resonance imaging}

CMR (1.5 Tesla, Philips Intera, Philips Medical Systems, Best, The Netherlands) was performed $5 \pm 2$ days (range 2-10) and $104 \pm 11$ days (range 75-122) after admis- 
sion. Images were ECG-gated and acquired during multiple breathholds using a dedicated 5-element phased array surface coil. Cine images were acquired in short axis views, completely covering the left ventricle, with a steady-state free precession sequence (average repetition time (TR) and echo time (TE) 3.8/1.9 ms respectively, flip angle $50^{\circ}$; slice thickness $6 \mathrm{~mm}$, gap $4 \mathrm{~mm}$, FOV $350 \mathrm{~mm}$, matrix 256 x 256, 2225 phases).

Delayed enhancement images (DE) were obtained 10 minutes after the intravenous administration of $0.2 \mathrm{mmol} / \mathrm{kg}$ body weight Gadolinium-diethylenetriaminepentaacetic acid (Magnevist ${ }^{\circledR}$, Schering, Germany) in short and two orthogonal long axis views, completely covering the left ventricle, using a $3 \mathrm{D}$ inversion recovery gradient-echo sequence (average TR/TE 3.9/2.4 ms, flip angle $15^{\circ}$; FOV $400 \mathrm{~mm}$, matrix $256 \times 256$, acquired and reconstructed voxel size $1.56 \times 1.56 \mathrm{~mm} \times 6 \mathrm{~mm}$ ). Immediately before DE imaging, a Look-Locker sequence was run to determine the inversion time (typical range 200-280 ms) to optimally suppress the signal of noninfarcted myocardium.

CMR images were analyzed off-line by a single experienced reader using commercially available software (CAAS MRV 3.0, Pie Medical Imaging, Maastricht, The Netherlands) blinded to the clinical and ECG data. Endocardial and epicardial borders were manually traced, excluding the papillary muscles, in the end-diastolic and end-systolic short-axis frames to determine end-systolic volume (LVESV), left ventricular stroke volume (LVSV), left ventricular ejection fraction (LVEF) and left ventricular end-diastolic mass (LV mass). These parameters were indexed to body surface area (BSA). Likewise, endocardial and epicardial contours were manually traced on the DE images. IS was quantified using a SI threshold of $>5$ SD above a remote noninfarcted region, including areas of MVO. When present, MVO was quantified by manually tracing the central hypoenhanced area within the hyperenhanced infarcted area. Both IS and MVO were expressed as a percentage of LV mass. The transmural extent of infarction was calculated by dividing the hyperenhanced area by the total area of the infarcted segment.

\section{Statistical analysis}

Multivariate techniques were used to analyze CMR and ECG variables separately. Subsequently, the predictive values of CMR variables on LV function at baseline and follow-up was conducted with a mixed linear regression model.

$K$-means cluster analyses were performed to identify three different subgroups of patients according to CMR based infarct characteristics, known to be associated with adverse LV remodeling and prognosis. ${ }^{7,13,14}$ The number of clusters $k=3$ was selected as a compromise between a sufficiently large number to describe different CMR groups and the limited sample size. Two cluster classification models were used: 1) clustering based on MVO (\%) and IS (\%) and 2) based on MVO (\%), IS (\%) 
and transmurality (\%). Larger average differences of LVEF among groups were regarded as indicative of better risk stratification. The classification model that best predicted adverse LV remodeling (low LVEF at follow-up) was then used to correlate with the STR variables. Crude associations between different groups, clinical and ST variables were subsequently tested via one-way ANOVA.

A stepwise discriminant analysis was carried out to test which of the individual STR variables were sufficiently powerful to discriminate among the defined groups. A complementary analysis of the independent effect of the CMR variables on the most discriminative ST measurement was performed via a linear regression model. Data analysis was conducted with SPSS version 18.0 (SPSS Inc, Chicago, IL) and SAS version 9.2 (SAS Institute, Cary, NC). Multiple testing correction, when necessary, was made via False Discovery Rate. Significance level was $5 \%$ for all tests.

\section{Results}

\section{Clustering based on CMR characteristics}

$K$-means cluster analysis identified three groups that differed significantly in their $\mathrm{CMR}$ risk profiles. First, patients were clustered based on MVO and IS (clustering model 1, Figure 1A) and second, based on MVO, IS and transmurality (clustering model 2, Figure 2A). In both figures, two more extreme groups can be identified that are quite antagonistic in their CMR risk profiles (high risk (red cluster) and low risk (green cluster)). The low risk group consisted of patients with either absent or small MVO, small (Figure $1 \mathrm{~A}$ ) and less transmural infarcts (figure 2A), whereas the opposite applies for the high risk group. Although the blue cluster is to a great extent an intermediate risk group, in terms of LV remodeling it closely resembled the low risk group (green cluster). The observed pair-wise correlations were $0.5,0.66$ and 0.34 for MVO/IS, transmurality/IS and MVO/transmurality, respectively $(p<0.05$ for all). Note that some patients had small but transmural infarcts with absent or small MVO (clustering model 1, green cluster, Figure 2A).

A comparative inspection of Figures $1 B$ and $2 B$ indicated that a larger average LVEF difference among the groups was observed in clustering model 1 (based on MVO and IS) than in model 2 (based on MVO, IS and transmurality). Thus, risk stratification was not improved by taking transmurality into account. The larger mean differences in model 1 were confirmed by the mixed modeling approach. The estimates of the fixed effect parameters of the random intercept model testing the independent effects of CMR profiles on LVEF over time are displayed in Table 1 (random intercept and residual variances were 28.84 and 15.46, respectively). LVEF in the high risk group remained significantly different from the other two risk groups both at baseline and follow-up. Henceforth, further inferences relate only to cluster- 
ing model 1 (based on MVO/IS clustering). CMR images of representative patient examples for the three risk groups are shown in Figure 3.
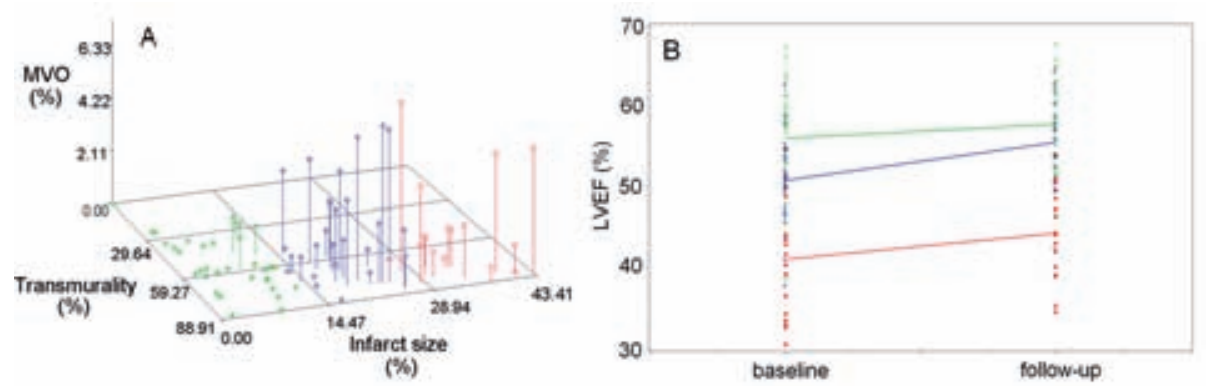

Figure 1. Cluster model 1 as a function of MVO and infarct size (A); left ventricular ejection fraction (LVEF) over time (from baseline to follow-up) for each of the three CMR risk groups (B). Red=high risk, blue=intermediate and green=low risk.
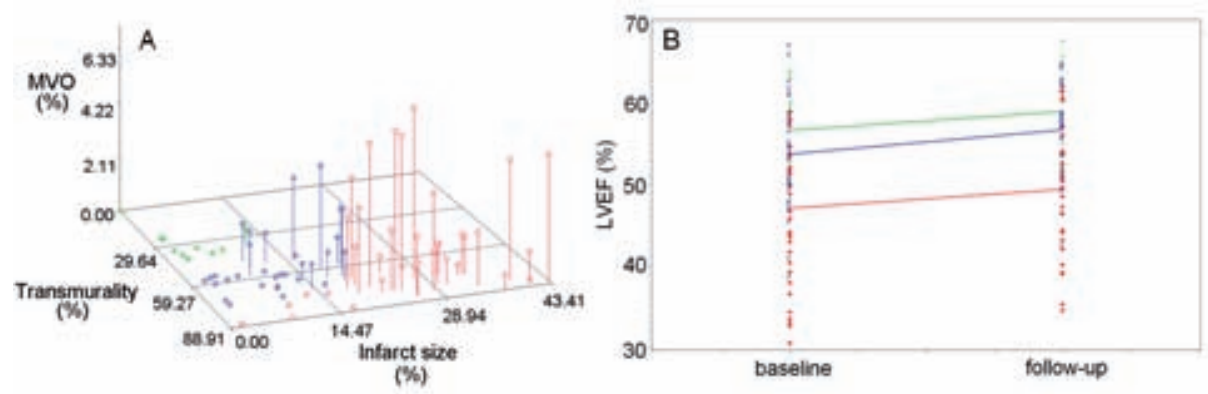

Figure 2. Cluster model 2 as a function of MVO, infarct size and transmurality (A); left ventricular ejection fraction (LVEF) over time (from baseline to follow-up) for each of the three CMR risk groups (B). Red=high risk, blue=intermediate and green=low risk.

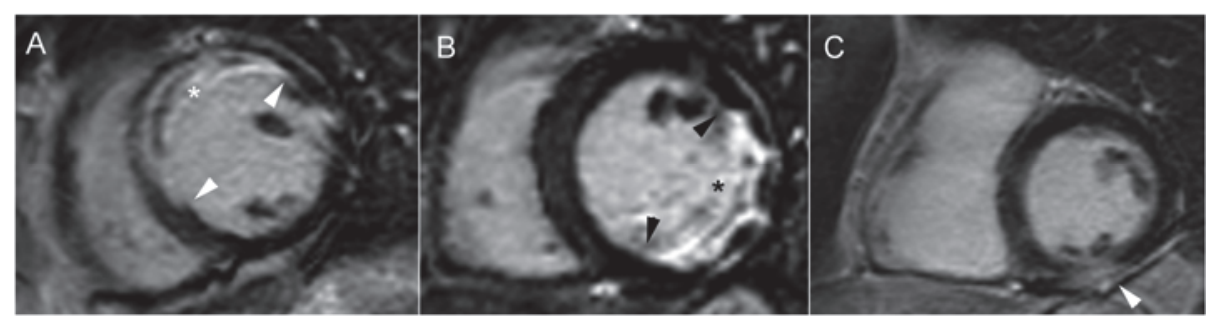

Figure 3. Delayed enhancement short axis slices of three representative patients from the high (panel A), intermediate (panel B) and low risk group (panel C). The hyperenhanced infarcted myocardium (arrowheads) and MVO $\left({ }^{*}\right)$ are shown. While patients $\mathrm{A}$ and $\mathrm{B}$ have similar mean infarct transmurality and MVO (62\% and $4.8 \%$ vs. $59 \%$ and $3.9 \%)$, infarct size in patient $A$ was larger than in patient B (32.2\% vs. $17.5 \%)$. Patient $\mathrm{C}$ had a small $(5.4 \%)$, but transmural (87\%) inferior wall infarct and no MVO. 
Table 1. Fixed effect parameters of the multivariable random intercept model with LVEF as outcome (based on cluster model 1).

\begin{tabular}{llll}
\hline & $\hat{\beta}$ & SE & $p$-value \\
\hline $\begin{array}{l}\text { Intercept } \\
\text { CMR risk group }\end{array}$ & 46.98 & 2.41 & $<0.0001$ \\
$\quad$ Low risk & 13.12 & 2.02 & $<0.0001$ \\
$\quad$ Intermediate risk & 9.23 & 2.07 & $<0.0001$ \\
$\quad$ High risk & 0 (reference) & 0 & \\
Time point 1 & -2.69 & 0.66 & $<0.0001$ \\
Time point 2 & 0 (reference) & 0 & \\
& & & \\
Baseline characteristic & & & n.s. \\
$\quad$ Sex (male) & -2.22 & 1.52 & n.s. \\
$\quad$ Anterior localisation & -1.67 & 1.64 & n.s. \\
Final TIMI 3 flow & 1.92 & 2.13 & \\
\hline$\hat{\beta}$ indicates an average change in LVEF for one unit increase in explanatory variable.
\end{tabular}

Abbreviations: LVEF=left ventricular ejection fraction; $C M R=$ cardiovascular magnetic resonance imaging; TIMI=Thrombolysis In Myocardial Infarction.

\section{Differences between CMR risk groups}

Patients in the high risk group ( $n=16$, large IS and MVO) were more likely to have an anterior $\mathrm{MI}$ and less likely to have baseline and final TIMI 3 flow as compared to patients with an intermediate $(n=28)$ and low risk CMR profile $(n=35$, Table 2$)$. Baseline LVESV $V_{i}$ was largest and LVEF lowest in the high risk group as compared to the

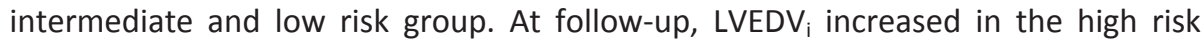
group and was significantly larger as compared to the intermediate and low risk group ( $99 \pm 19 \mathrm{ml} / \mathrm{m}^{2}, 79 \pm 15 \mathrm{ml} / \mathrm{m}^{2}, 77 \pm 15 \mathrm{ml} / \mathrm{m}^{2}$, respectively, $p=0.002$ ). Average LVEF increased in every risk group, but more so in the intermediate risk group, leading to a loss of the initial difference in LVEF at baseline between the intermediate and low risk group. Note that LVEF in the intermediate risk group was on average higher than in the high risk group, despite a similar prevalence and extent of MVO ( $82 \%$ vs. $94 \%$ and $1.9 \pm 1.8 \%$ vs. $1.9 \pm 1.9 \%$, respectively). At follow-up, LVEF remained lowest in the high risk group as compared to the intermediate and low risk group ( $44 \pm 6 \%, 56 \pm 5 \%, 58 \pm 5 \%$, respectively, $p=0.002$ ).

STR (either summed or single lead) was smallest in the high risk group as compared to the intermediate and low risk group. This was only significant for the 30 minute time point. Residual STD (either summed or worst lead) was significantly higher in the high risk group as compared to the other groups for the 30 and 60 minute time point. 
Table 2. Comparison of clinical characteristics between CMR risk groups.

\begin{tabular}{|c|c|c|c|c|}
\hline & \multicolumn{3}{|c|}{ CMR risk groups } & \multirow[b]{2}{*}{$\begin{array}{l}\text { FDR } \\
p \text {-value }\end{array}$} \\
\hline & $\begin{array}{l}\text { High risk } \\
(n=16)\end{array}$ & $\begin{array}{l}\text { Intermediate } \\
\text { risk }(n=28)\end{array}$ & $\begin{array}{l}\text { Low risk } \\
(\mathrm{n}=35)\end{array}$ & \\
\hline Age (years) & $63 \pm 15$ & $58 \pm 12$ & $59 \pm 9$ & 0.46 \\
\hline Male (\%) & 88 & 57 & 83 & 0.045 \\
\hline \multicolumn{5}{|l|}{ Risk factors } \\
\hline Diabetes Mellitus (\%) & 0 & 0 & 9 & 0.19 \\
\hline Hyperlipidemia (\%) & 31 & 37 & 40 & 0.85 \\
\hline Hypertension (\%) & 38 & 30 & 43 & 0.65 \\
\hline Smoking (\%) & 81 & 93 & 89 & 0.57 \\
\hline Anterior MI (\%) & 88 & 25 & 14 & 0.002 \\
\hline Glycoprotein 2b3a inhibition (\%) & 63 & 57 & 49 & 0.65 \\
\hline Thrombosuction (\%) & 31 & 25 & 36 & 0.77 \\
\hline \multicolumn{5}{|l|}{ TIMI 3 flow } \\
\hline Baseline (\%) & 0 & 0 & 17 & 0.045 \\
\hline Final (\%) & 75 & 100 & 88 & 0.045 \\
\hline Ischemic time (min.) & $222 \pm 87$ & $231 \pm 72$ & $216 \pm 91$ & 0.77 \\
\hline \multicolumn{5}{|l|}{ Baseline CMR } \\
\hline Infarct size (\% of LV) & $34 \pm 5^{*}{ }^{+}$ & $19 \pm 4^{* \prime}$ & $6 \pm 4^{+, \neq}$ & 0.002 \\
\hline Transmurality (\%) & $79 \pm 7^{*}$ & $71 \pm 10^{+}$ & $52 \pm 21^{*+}$ & 0.002 \\
\hline MVO prevalence (\%) & $94 *$ & $82^{+}$ & $17^{*,^{+}}$ & 0.002 \\
\hline MVO size (\% of LV) & $1.9 \pm 1.9 *$ & $1.9 \pm 1.8^{+}$ & $0.2 \pm 0.4^{*+}$ & 0.002 \\
\hline $\operatorname{LVEDV}_{\mathrm{i}}\left(\mathrm{ml} / \mathrm{m}^{2}\right)$ & $91 \pm 20$ & $83 \pm 14$ & $82 \pm 14$ & 0.19 \\
\hline $\operatorname{LVESV}_{\mathrm{i}}\left(\mathrm{ml} / \mathrm{m}^{2}\right)$ & $54 \pm 15^{*^{+}}$ & $41 \pm 10^{*}$ & $37 \pm 11^{+}$ & 0.002 \\
\hline LVEF (\%) & $41 \pm 7^{*+}$ & $51 \pm 5^{*, \ddagger}$ & $56 \pm 7^{+, \neq}$ & \\
\hline \multicolumn{5}{|l|}{ Follow-up CMR } \\
\hline $\operatorname{LVEDV}_{\mathrm{i}}\left(\mathrm{ml} / \mathrm{m}^{2}\right)$ & $99 \pm 19^{*{ }^{+}}$ & $79 \pm 15^{*}$ & $77 \pm 15^{+}$ & 0.002 \\
\hline $\operatorname{LVESV}_{\mathrm{i}}\left(\mathrm{ml} / \mathrm{m}^{2}\right)$ & $56 \pm 15^{*+}$ & $35 \pm 9 *$ & $32 \pm 9^{+}$ & 0.002 \\
\hline $\operatorname{LVEF}(\%)$ & $44 \pm 6^{*{ }^{+}}$ & $56 \pm 5^{*}$ & $58 \pm 5^{+}$ & 0.002 \\
\hline $\operatorname{LVEDV}_{\mathrm{i}}\left(\mathrm{ml} / \mathrm{m}^{2}\right)$ & $99 \pm 19^{*,^{+}}$ & $79 \pm 15^{*}$ & $77 \pm 15^{+}$ & 0.002 \\
\hline CK AUC $\left(1,000\right.$ h.units $\left.I^{-1}\right)$ & $94 \pm 34^{*+}$ & $57 \pm 27^{* \prime \neq}$ & $29 \pm 24^{+, \neq}$ & 0.002 \\
\hline \multicolumn{5}{|l|}{ ECG variables } \\
\hline$\Sigma S T R(30)(\%)$ & $42 \pm 27^{*{ }^{+}}$ & $66 \pm 26^{*}$ & $72 \pm 25^{+}$ & 0.007 \\
\hline$\Sigma \mathrm{STR}(60)(\%)$ & $57 \pm 23$ & $74 \pm 25$ & $70 \pm 29$ & 0.17 \\
\hline $\operatorname{STR}(30)(\%)$ & $49 \pm 28^{*+}$ & $68 \pm 24^{*}$ & $74 \pm 25^{+}$ & 0.003 \\
\hline $\operatorname{STR}(60)(\%)$ & $62 \pm 22$ & $75 \pm 25$ & $75 \pm 26$ & 0.23 \\
\hline$\Sigma \mathrm{STD}(30)(\mathrm{mm})$ & $9.8 \pm 5^{*^{+}}$ & $4.4 \pm 3.1^{*}$ & $3.2 \pm 3.5^{\dagger}$ & 0.002 \\
\hline$\Sigma \mathrm{STD}(60)(\mathrm{mm})$ & $7.4 \pm 3.9^{*+}+$ & $3.2 \pm 2.7^{*}$ & $2.9 \pm 2.6^{\dagger}$ & 0.002 \\
\hline $\mathrm{STD}(30)(\mathrm{mm})$ & $2.6 \pm 1.5^{*{ }^{+}}$ & $1.2 \pm 0.9 *$ & $0.8 \pm 0.7^{\dagger}$ & 0.002 \\
\hline $\mathrm{STD}(60)(\mathrm{mm})$ & $2 \pm 1.4^{*,^{\dagger}}$ & $0.9 \pm 0.9 *$ & $0.7 \pm 0.6^{\dagger}$ & 0.002 \\
\hline
\end{tabular}

Values are presented as percentages in parentheses or mean \pm standard deviation. Abbreviations: Ml=myocardial infarction, TIMI=thrombolysis in myocardial infarction; $\mathrm{CMR}=$ cardiovascular magnetic resonance imaging; MVO=microvascular obstruction; $L_{V E D V}=$ left ventricular end-diastolic volume index; LVESV $_{i}=$ left ventricular end-systolic volume index; LVEF=left ventricular ejection fraction; CK=creatine kinase; $A U C=a r e a$ under the curve; STR=ST segment resolution; STD=ST segment deviation; $\Sigma$ =summed (otherwise single lead is meant); (30), $(60)=30$ and 60 minutes post$\mathrm{PCl} ; \mathrm{FDR}=$ false discovery rate; The superscripts $\left({ }^{*,+, \neq}\right)$ indicate significant pairwise group comparison. 


\section{CMR and ST segment variables: stepwise discriminant analysis}

With the objective to identify which of the STR variables were more useful in separating the different $\mathrm{CMR}$ risk groups, a stepwise discriminant analysis was carried out. Only worst lead residual ST deviation 30 minutes post-PCI (STD (30)) was found to separate, though poorly, the three risk groups (classification and leave-one out cross-validation rates were both 55\%).

Because the intermediate and low risk group were on average closer to each other with respect to LV remodeling (Figure 1B), they were joined ad hoc to form one group. Following a new discriminant analysis, STD (30) remained the sole discriminatory variable between the two groups (high vs. low/intermediate), but now the classification rate was considerably improved ( $82 \%$ cross-validated classification). The AUC under the ROC curve was 0.86 , with $95 \% \mathrm{Cl} 0.77$ and 0.96 , respectively. Because of small numbers, it was not possible to define STD cutoff values for anterior and non-anterior MI separately, but for the whole group a STD cutoff value of $1.53 \mathrm{~mm}$ gave a sensitivity of $88 \%$ and specificity of $76 \%$. Of the CMR variables (IS, MVO and transmurality) tested in a multivariable linear regression model, only IS was shown to be significant associated with STD (Table 3).

\section{Discussion}

The current study shows that worst lead residual STD 30 minutes after reperfusion allows accurate identification of patients at high risk for adverse remodeling after 3 months, which was mainly related to IS rather than transmurality or MVO.

Studies investigating the role of infarct characteristics on functional recovery and LV remodeling after AMI have been inconclusive, indicating that it is a heterogeneous process hitherto incompletely understood. Some studies have favored infarct transmurality, whereas others have found either IS or MVO to be independently associated with adverse remodeling or outcome. ${ }^{7,12-14}$ These studies primarily focused on univariate procedures (either uni-or multivariable) to evaluate the prognostic value of aforementioned infarct characteristics. Although univariate techniques may show independent effects that can be combined in additive models, they offer only a piece-wise perspective into relationships and a broader picture may still be missed. Alternatively, to the one-variable at a time analysis, we explored the correlational information among the CMR variables by clustering patients into three different risk groups. This approach revealed that IS accounts for the most conspicuous average difference among the risk profiles with respect to their recovery of LV function over time. 
The downgraded role of the last two CMR parameters is at odds with previous reports. Our small-scale study does not provide a definite answer to this contentious topic. Our findings may draw attention to alternative clinical hypotheses that may explain why risk stratification was not improved, once transmurality was added to the clustering model. Functional impairment after a large AMI is not only related to myocyte loss and inflammatory changes in the infarcted myocardium itself. Increased systolic work load on remote myocardium also contributes significantly to the loss in global ventricular function. The impact of remote myocardial dysfunction in small infarcts is minimal. Even if they are transmural, these small infarcts retain the ability to recover over time (Figure $3 \mathrm{C}$ ). ${ }^{16}$

The relation of MVO and myocardial necrosis is still unclear. The concept that reperfusion injury may increase final IS due to death of additional myocytes, still viable immediately before reperfusion, is questionable. ${ }^{17} \mathrm{~A}$ solid argument against a causal role of MVO for infarct progression during reperfusion is that MVO lags behind myocyte death. ${ }^{18}$ Experimental studies have shown a strong correlation between MVO and IS. ${ }^{19}$ If this correlation holds, any predictive role that MVO may have on adverse outcome is potentially confounded by IS. Up to date, only a few investigations have been able to demonstrate an independent effect of MVO on poor prognosis, i.e. once adjusted for IS. However, consensus has not yet been established and MVO's independent prognostic role remains moot. ${ }^{19}$ The present study does not reinforce this hypothesis. A positive, though moderate correlation of MVO with IS was observed. The correlation was especially weak for the high risk group, where small and high MVO values were linked with large IS (explaining why MVO was similar in the intermediate and high risk CMR group). This finding lends more support to a predictive role of IS rather than MVO on adverse remodeling. STR is widely used as a marker for myocardial reperfusion, irrespective of the degree of antegrade epicardial coronary flow. Its well established prognostic value ${ }^{20,21}$ may be explained by its relation with IS rather than MVO.

The association of STR with IS and long-term ventricular function has been previously reported, but, in contrast to the current study, the influence of MVO was not investigated. ${ }^{10,22}$ We found that STR could not discriminate satisfactorily among the three CMR risk groups. The discriminatory power increased considerably, when it came to separating between high vs. the combined low/intermediate risk groups. Analogous to the LVEF behavior, this suggests that the intermediate and low risk groups constitute a more homogeneous group with respect to STR, despite showing statistically significant differences in all three CMR parameters. Intermediate and high risk patients, on the other hand, were similar in their MVO and transmurality characteristics, differing only in IS. Thus, IS emerges, by exclusion, as the major factor affecting STR rather than MVO or transmurality. This conclusion is supported by the results of the multivariable regression model, with IS as the sole remaining CMR variable significantly associated with STD. 
Investigators have used a variety of different techniques to measure STR, either single or multiple lead and either resolution or worst lead residual STD after reperfusion. The latter is by far the simplest and clinically most applicable method for calculating STR. ${ }^{23}$ Our results show that worst lead STD measured as early as 30 minutes after reperfusion is at least as accurate as all other ST variables in predicting adverse remodeling and identifying patients at high risk for adverse remodeling.

\section{Conclusions}

Worst lead residual STD 30 minutes after reperfusion accurately identifies patients at high risk for adverse remodeling after 3 months, which was mainly related to IS rather than transmurality or MVO. 


\section{References}

1. De Luca G, van 't Hof AW, de Boer MJ, Ottervanger JP, Hoorntje JC, Gosselink AT, Dambrink JH, Zijlstra F, Suryapranata H. Time-to-treatment significantly affects the extent of ST-segment resolution and myocardial blush in patients with acute myocardial infarction treated by primary angioplasty. Eur Heart J 2004;25:1009-13.

2. Ito H, Tomooka T, Sakai N, Yu H, Higashino Y, Fujii K, Masuyama T, Kitabatake A, Minamino T. Lack of myocardial perfusion immediately after successful thrombolysis. A predictor of poor recovery of left ventricular function in anterior myocardial infarction. Circulation 1992;85:1699-705.

3. Kloner RA, Ganote CE, Jennings RB. The "no-reflow" phenomenon after temporary coronary occlusion in the dog. J Clin Invest 1974;54:1496-508.

4. de Lemos JA, Antman EM, Giugliano RP, McCabe CH, Murphy SA, Van de Werf F, Gibson CM, Braunwald E. ST-segment resolution and infarct-related artery patency and flow after thrombolytic therapy. Thrombolysis in Myocardial Infarction (TIMI) 14 investigators. Am J Cardiol 2000;85:299304.

5. Amaya N, Nakano A, Uzui H, Mitsuke Y, Geshi T, Okazawa H, Ueda T, Lee JD. Relationship between microcirculatory dysfunction and resolution of ST-segment elevation in the early phase after primary angioplasty in patients with ST-segment elevation myocardial infarction. Int J Cardiol 2010.

6. Feldman LJ, Coste P, Furber A, Dupouy P, Slama MS, Monassier JP, Tron C, Lafont A, Faraggi M, Le Guludec D, Dubois-Rande JL, Steg PG. Incomplete resolution of ST-segment elevation is a marker of transient microcirculatory dysfunction after stenting for acute myocardial infarction. Circulation 2003;107:2684-9.

7. Nijveldt R, Beek AM, Hirsch A, Stoel MG, Hofman MB, Umans VA, Algra PR, Twisk JW, van Rossum AC. Functional recovery after acute myocardial infarction: comparison between angiography, electrocardiography, and cardiovascular magnetic resonance measures of microvascular injury. J Am Coll Cardiol 2008;52:181-9.

8. Santoro GM, Valenti R, Buonamici P, Bolognese L, Cerisano G, Moschi G, Trapani M, Antoniucci D, Fazzini PF. Relation between ST-segment changes and myocardial perfusion evaluated by myocardial contrast echocardiography in patients with acute myocardial infarction treated with direct angioplasty. Am J Cardiol 1998;82:932-7.

9. Buller CE, Fu Y, Mahaffey KW, Todaro TG, Adams P, Westerhout CM, White HD, van 't Hof AW, Van de Werf FJ, Wagner GS, Granger CB, Armstrong PW. ST-segment recovery and outcome after primary percutaneous coronary intervention for ST-elevation myocardial infarction: insights from the Assessment of Pexelizumab in Acute Myocardial Infarction (APEX-AMI) trial. Circulation 2008;118:1335-46.

10. Dong J, Ndrepepa G, Schmitt C, Mehilli J, Schmieder S, Schwaiger M, Schomig A, Kastrati A. Early resolution of ST-segment elevation correlates with myocardial salvage assessed by Tc-99m sestamibi scintigraphy in patients with acute myocardial infarction after mechanical or thrombolytic reperfusion therapy. Circulation 2002;105:2946-9.

11. Sciagra R, Parodi G, Migliorini A, Valenti R, Antoniucci D, Sotgia B, Pupi A. ST-segment analysis to predict infarct size and functional outcome in acute myocardial infarction treated with primary coronary intervention and adjunctive abciximab therapy. Am J Cardiol 2006;97:48-54.

12. Larose E, Rodes-Cabau J, Pibarot P, Rinfret S, Proulx G, Nguyen CM, Dery JP, Gleeton O, Roy L, Noel B, Barbeau G, Rouleau J, Boudreault JR, Amyot M, De Larochelliere R, Bertrand OF. Predicting late myocardial recovery and outcomes in the early hours of ST-segment elevation myocardial infarction traditional measures compared with microvascular obstruction, salvaged myocardium, and necrosis characteristics by cardiovascular magnetic resonance. J Am Coll Cardiol 2010;55:2459-69.

13. Tarantini G, Razzolini R, Cacciavillani L, Bilato C, Sarais C, Corbetti F, Marra MP, Napodano M, Ramondo A, lliceto $S$. Influence of transmurality, infarct size, and severe microvascular obstruction on left ventricular remodeling and function after primary coronary angioplasty. Am J Cardiol 2006;98:1033-40. 
14. Choi KM, Kim RJ, Gubernikoff G, Vargas JD, Parker M, Judd RM. Transmural extent of acute myocardial infarction predicts long-term improvement in contractile function. Circulation 2001;104:1101-7.

15. Kim HW, Farzaneh-Far A, Kim RJ. Cardiovascular magnetic resonance in patients with myocardial infarction: current and emerging applications. J Am Coll Cardiol 2009;55:1-16.

16. Bogaert J, Bosmans H, Maes A, Suetens P, Marchal G, Rademakers FE. Remote myocardial dysfunction after acute anterior myocardial infarction: impact of left ventricular shape on regional function: a magnetic resonance myocardial tagging study. J Am Coll Cardiol 2000;35:1525-34.

17. Yellon DM, Hausenloy DJ. Myocardial reperfusion injury. N Engl J Med 2007;357:1121-35.

18. Fishbein MC, J YR, Lando U, Kanmatsuse K, Mercier JC, Ganz W. The relationship of vascular injury and myocardial hemorrhage to necrosis after reperfusion. Circulation 1980;62:1274-9.

19. Reffelmann T, Hale SL, Li G, Kloner RA. Relationship between no reflow and infarct size as influenced by the duration of ischemia and reperfusion. Am J Physiol Heart Circ Physiol 2002;282:H766-72.

20. Schroder R, Wegscheider K, Schroder K, Dissmann R, Meyer-Sabellek W. Extent of early ST segment elevation resolution: a strong predictor of outcome in patients with acute myocardial infarction and a sensitive measure to compare thrombolytic regimens. A substudy of the International Joint Efficacy Comparison of Thrombolytics (INJECT) trial. J Am Coll Cardiol 1995;26:1657-64.

21. van 't Hof AW, Liem A, de Boer MJ, Zijlstra F. Clinical value of 12-lead electrocardiogram after successful reperfusion therapy for acute myocardial infarction. Zwolle Myocardial infarction Study Group. Lancet 1997;350:615-9.

22. Thiele H, Kappl MJ, Linke A, Erbs S, Boudriot E, Lembcke A, Kivelitz D, Schuler G. Influence of timeto-treatment, TIMI-flow grades, and ST-segment resolution on infarct size and infarct transmurality as assessed by delayed enhancement magnetic resonance imaging. Eur Heart J 2007;28:1433-9.

23. de Lemos JA, Braunwald E. ST segment resolution as a tool for assessing the efficacy of reperfusion therapy. J Am Coll Cardiol 2001;38:1283-94. 


\title{
CHAPTER 6
}

\author{
The performance of angiographic, \\ electrocardiographic and, magnetic \\ resonance imaging methods to assess \\ the area at risk in acute myocardial \\ infarction
}

Versteylen MO, Bekkers SCAM, Smulders MW, Winkens B, Mihl C, Winkens MHM, Leiner T, Waltenberger JL, Kim RJ, Gorgels APM 


\section{Abstract}

\section{Background}

Validation of area at risk (AAR) measurement methods in acute myocardial infarction (AMI) in humans is limited. We tested the performance of different AAR measurement methods in AMI using established physiologic concepts to provide a reference standard.

\section{Methods and results}

In 78 percutaneously reperfused first ST-elevation myocardial infarction patients, AAR was measured by electrocardiographic (Aldrich), angiographic (BARI, AP$\mathrm{PROACH}$ ), and cardiovascular magnetic resonance (CMR) methods (T2-weighted (T2W) hyperintensity, delayed enhanced (DE) endocardial surface area (ESA)). Infarct size (IS) and transmurality were measured by DE-CMR. The following established physiologic concepts were used to evaluate AAR methods: 1) AAR should be greater than or equal to IS, 2) in transmural infarcts, AAR should approach IS, 3) the correlation between AAR and IS should increase as infarct transmurality increases, 4) myocardial salvage [(AAR-IS)/AAR $\times 100]$ should be inversely related to transmurality, following the inverse line of identity. Overall, $65 \%, 87 \%, 76 \%, 87 \%$, and $97 \%$ of patients using Aldrich, BARI, APPROACH, T2W, and ESA obeyed the concept $A A R \geq I S$. In a sub-cohort of patients $(n=22)$ with near transmural infarcts $(>75 \%)$, the 95\% limits of agreement on Bland-Altman analysis were -22.9 to $29.6,-28.3$ to 21.3 , -16.9 to $20.0,-6.9$ to 16.6 and -4.3 to 18.0 for BARI, Aldrich, APPROACH, T2W and ESA, respectively. Increasing correlation with increasing transmurality was only observed for APPROACH, T2W and ESA, with ESA having the highest correlation in the nearly transmural sub-cohort $(r=0.93, p<0.001)$. Concerning salvage and infarct transmurality, the percentage of patients within a narrow margin $( \pm 30 \%)$ of the inverse line of identity (high=better) were $56 \%, 76 \%, 65 \%, 77 \%$ and $92 \%$ for Aldrich, BARI, APPROACH, T2W and ESA.

\section{Conclusions}

For measuring AAR, CMR methods were better than angiographic methods, which were better than electrocardiographic. Overall, ESA by DE-CMR appeared to be the best method for measuring AAR in vivo. 


\section{Introduction}

Clinical outcome after acute myocardial infarction (MI) largely depends on final infarct size (IS) and is improved by early reperfusion. ${ }^{1}$ IS is determined by the extent of collateral flow, myocardial metabolic demand during occlusion, duration of coronary occlusion and size of the initial area at risk (AAR). ${ }^{2,3}$ Determining both AAR and IS allows the assessment of myocardial salvage and evaluation of the efficacy of reperfusion strategies.

Delayed enhanced cardiovascular magnetic resonance imaging (DE-CMR) reliably detects IS and accurately distinguishes between viable and nonviable myocardium. ${ }^{4,5}$ However, determination of the AAR in the clinical setting remains challenging. Although single-photon emission computed tomographic (SPECT) is an accepted technique ${ }^{6,7}$, radiation exposure, low spatial resolution and difficult logistics requiring tracer injection before reperfusion complicate this method.

STEMI diagnosis is supported by the electrocardiogram (ECG) that is routinely performed on admission and most patients will undergo coronary angiography followed by percutaneous coronary intervention (PCI). Both electrocardiographic and coronary angiographic measures have previously been used to measure the AAR. The Aldrich score is based on the total amount of ST-segment deviation on admission ${ }^{8}$, whereas BARI and APPROACH are based on vessel anatomy and location of coronary obstruction. ${ }^{9,10}$ Myocardial ischemia leads to inflammation and tissue edema in the infarct related artery (IRA) territory. ${ }^{11}$ This edema is shown as an area of increased signal intensity on T2 weighted (T2W) CMR and animal studies suggest that this hyperintense area delineates the AAR in STEMI. ${ }^{12,13}$ More recently, endocardial surface area (ESA) on DE-CMR was used for measuring AAR. ${ }^{14,15}$ Although promising, for all the proposed methods the number of studies is small and more validation in humans with appropriate reference standards is necessary. ${ }^{16,17}$

In this study electrocardiographic, angiographic, and CMR techniques for measuring AAR were evaluated using established and straightforward physiologic concepts based on the "wavefront phenomenon" of infarct progression from endocardium to epicardium with only minimal lateral extension. ${ }^{2}$

\section{Methods}

\section{Patients}

The study was approved by the Institutional Review Board of our hospital and patients were included after written informed consent was obtained. Between August 2006 and March 2008, 106 consecutive patients with a first ST segment elevation myocardial infarction (STEMI) referred to our institution for primary $\mathrm{PCl}$ and presen- 
tation $<12$ hours after symptom onset were prospectively studied. Incomplete datasets in 28 patients (coronary angiography in 8, ECG in 9 and T2W in 11 patients) were excluded and this study comprises the results of 78 patients. Definition of $\mathrm{MI}$ was based on the recent consensus document including appropriate rise and fall in cardiac biomarkers. ${ }^{18}$ Excluded were patients with claustrophobia, contraindications for CMR (cerebral clips, pacemakers, defibrillators, neurostimulators), severe congestive heart failure or cardiogenic shock (Killip class III and IV) at the time of $\mathrm{CMR}$, atrial fibrillation, age $<18$ years, severe renal failure (stage 4 or 5 ), and pregnancy.

\section{Coronary angiography}

After pre-treatment with aspirin, heparin and, clopidogrel, patients underwent standard catheterization with multiple selective contrast injections in the left and right coronary arteries prior to $\mathrm{PCl}$. Thrombosuction and downstream administration of intravenous antiplatelet agents (abciximab), intracoronary nitroglycerine and adenosine were left at the discretion of the interventional cardiologist. $\mathrm{PCl}$ was performed in all patients and every patient received at least one stent.

Two observers, blinded to the electrocardiographic and CMR data, independently reviewed all angiograms. Consensus was reached by discussion in all patients. Collaterals were defined by visual assessment using the Rentrop classification (19). Antegrade flow in the IRA before and after PCI was characterized using the Thrombolysis In Myocardial Infarction (TIMI) scoring system. ${ }^{20}$

\section{Angiographic AAR assessment}

Angiographic AAR measurement was performed as previously described. ${ }^{9}$ In this study, we calculated the AAR for the IRA.

\section{BARI-score}

Using the Bypass Angioplasty Revascularization Investigation Myocardial Jeopardy Index (BARI), AAR was calculated by grading all terminating arteries. ${ }^{9}$ All branches were scored 3, 2, 1 or 0 points, corresponding to large, medium, small or absent. The ventricular base to apex distance, approximated from the coronary angiogram, provided the basis for assessing the relative distribution of coronary branches. Branches were considered large if their length exceeded $2 / 3$, medium if $1 / 3$ to $2 / 3$, small if less than $1 / 3$ and absent if less than $1 / 5$ of the distance from base to apex. The AAR was calculated as a percentage of the left ventricle (LV) by dividing summed scores of a jeopardized area by the total score of the entire LV. 


\section{Modified APPROACH-score}

In the APPROACH score, the LV was divided into regions that represent the proportion of myocardium perfused by each coronary artery, which was estimated based on post-mortem pathology studies. ${ }^{21}$ In this study we used the modified APPROACH score. ${ }^{15}$ Scoring was performed using a template in which the culprit artery is selected, as well as proximity of the lesion and size of side branches (small/absent, medium or large). For left circumflex artery (LCX) and right coronary artery (RCA) culprit lesion, left- or right dominance was determined. The template than provided one of 39 possible AAR scores as a percentage of the LV.

\section{Electrocardiographic AAR assessment (Aldrich method)}

Two observers, blinded from the coronary angiography and CMR data, independently determined the Aldrich scores on the standard 12-lead admission ECG ${ }^{8}$. Using the TP segment as the isoelectric line, ST-elevation was measured manually, $60 \mathrm{~ms}$ after the J-point to the nearest $0.5 \mathrm{~mm}$ in each lead. Any ST-elevation $\geq 1 \mathrm{~mm}$ was measured in leads II, III, avF for inferior STEMI and in all leads for anterior STEMI. Electrocardiographic AAR was then calculated using the Aldrich score: anterior $\mathrm{MI}: 3 \times[1.5 \times($ number of leads with ST个) -0.4$]$; inferior MI: $3 \times[0.6 \times(\Sigma \mathrm{ST} \uparrow \mathrm{II}, \mathrm{III}$, $A V F)+2.0]$ and computed as a percentage of LV.

\section{Cardiovascular magnetic resonance imaging}

CMR was performed $5 \pm 2$ days after admission. Images were acquired during multiple breathholds on a 1.5 Tesla MRI system (Intera R 11.3, Philips Medical Systems, Best, The Netherlands) with a dedicated 5-element phased array surface coil. To image myocardial edema, a double inversion T2-weighted (T2W) turbo spin echo sequence with spectrally selective inversion recovery (SPIR) fat suppression was used in multiple contiguous short axis slices covering the entire LV (slice thickness 8 $\mathrm{mm}$, gap $2 \mathrm{~mm}$, TR/TE: 2 R-R intervals $/ 100 \mathrm{~ms}$, flip angle $90^{\circ}$, FOV $350 \mathrm{~mm}$, matrix $236 \times 186)$. DE was performed 10-15 minutes after administration of $0.2 \mathrm{mmol} / \mathrm{kg}$ body weight Gadolinium-diethylenetriaminepentaacetic acid (Magnevist ${ }^{\circledR}$, Bayer Schering Pharma, Berlin, Germany) using a 3D segmented inversion-recovery gradient-echo sequence (reconstructed slice thickness $6 \mathrm{~mm}$, average TR/TE 3.9/2.4 ms, flip angle $15^{\circ}$, FOV $400 \mathrm{~mm}$, matrix $256 \times 256$, reconstructed pixel size $1.56 \times$ $1.56 \mathrm{~mm}$ ). A Look-Locker sequence was used to find the inversion time that optimally suppressed the signal of non-infarcted myocardium (typical range 200-280 ms).

\section{CMR Image Analysis}

CMR images were analyzed off-line using commercially available software (CAAS MRV 3.0, Pie Medical Imaging, Maastricht, The Netherlands) and blinded to the 
electrocardiography and angiography results. Endocardial and epicardial contours were manually traced on the T2W and DE images.

The AAR was quantified on the T2W images by delineating myocardium with a signal intensity $(\mathrm{SI})$ threshold of $>2$ SD above the mean of a remote region outside the IRA territory. ${ }^{17}$ Areas of central hypoenhancement were included and endocardial 'slow flow' artifacts were excluded by manual adjustment of contours.

IS was quantified on the DE images by delineating areas of hyperenhancement using a SI threshold of $>5$ SD above the mean of a remote non-infarcted region, including areas of microvascular obstruction (MVO). ${ }^{22}$

AAR and IS were expressed as a percentage of LV mass. The transmural extent of infarction was calculated by dividing the hyperenhanced volume by the total volume of the infarcted segments. The infarct ESA was calculated as a percentage of the hyperenhanced endocardial surface area divided by total LV endocardial surface area (including MVO).

For each method, the data of 25 randomly selected patients were analyzed by one observer twice on two different occasions and a second observer independently analyzed all data, so that intra- and interobserver variability could be calculated.

\section{Physiologic concepts}

Patients were classified into three groups, based on the results of DE-CMR: a) subendocardial infarction (transmurality $<50 \%, n=13$ ), b) near transmural infarction (transmurality $>75 \%, \mathrm{n}=22$ ), and $\mathrm{c}$ ) intermediate transmurality (transmurality 50 $75 \%, n=43$ ). Each method was evaluated by using the following established physiologic concepts: 1) AAR should always be larger than or equal to IS; 2 ) in nearly transmural infarcts, AAR should approach IS; 3) as infarct transmurality increases, the correlation between AAR and IS should increase; 4) myocardial salvage [defined as AAR - IS)/AAR $\times 100$ ] should be inversely correlated with transmurality, closely following the inverse line of identity [salvage $=100 \%-$ transmurality]. The deviation from this 'line of identity' was expressed as percentage of patients within a $\pm 30 \%$ margin and in the standard error of estimate (SEE). Individual AAR methods were ranked ( 1 , worst to 5 , best) based on how well they agreed to these physiologic concepts.

\section{Statistical Analyses}

Continuous variables were presented as mean \pm standard deviations (SD) and proportions (\%) were used for categorical variables. In near transmural infarcts, agreement between AAR and IS was tested using Bland-Altman analysis, with reporting of bias and $95 \%$ confidence intervals. Correlations were computed using Pearson's correlation coefficient. The predictive accuracy of the AAR methods concerning the 
inverse relation between myocardial salvage and infarct transmurality was measured using the standard error of the estimate (SEE). A two-tailed $p$-value $\leq 0.05$ was considered statistically significant. The data were analyzed using SPSS 17.0 for Windows (SPSS Inc., Chicago, IL).

Table 1. Patient characteristics.

\begin{tabular}{ll}
\hline Characteristic & Value \\
\hline $\mathrm{N}$ & 78 \\
Age (years) & $59 \pm 11$ \\
Male (\%) & $55(71)$ \\
Current smoker (\%) & $43(49)$ \\
Diabetes (\%) & $5(6)$ \\
Hypertension (\%) & $29(37)$ \\
Hyperlipidemia (\%) & $21(24)$ \\
& \\
Infarct related artery (\%) & \\
$\quad$ LAD & $26(33)$ \\
$\quad$ RCA & $41(53)$ \\
$\quad$ LCx & $11(14)$ \\
No. of diseased vessels (\%) & \\
$\quad$ One & $40(51)$ \\
$\quad$ Two & $19(24)$ \\
Three & $19(25)$ \\
Symptom to balloon time (min.) & $218 \pm 83$ \\
Glycoprotein 2b3a inhibition & $40(51)$ \\
TIMI 3 flow & \\
Pre PCl & $95 \pm(12)$ \\
Post PCl & $71(91)$ \\
Infarcts size (\%) & \\
\hline Infarct transmurality (\%) & \\
\hline
\end{tabular}

Values are presented as numbers (\%) or means \pm standard deviation. Abbreviations: $\mathrm{LAD}=$ left anterior descending artery; RCA=right coronary artery; LCX=left circumflex coronary artery; TIMI=thrombolysis in myocardial infarction; $\mathrm{PCl}=$ percutaneous coronary intervention.

\section{Results}

Baseline characteristics are shown in Table 1. The majority of patients were men (71\%), approximately half of patients had single vessel disease (51\%) and in $53 \%$ the RCA was IRA. Final TIMI 3 flow was established in $91 \%$. Mean IS was $16 \pm 11 \%$ of LV mass. A trend in decreasing pre-PCI TIMI flow was observed with increasing trans- 
murality $(p=0.07)$. Collaterals and symptom-to-balloon-time were not different between subgroups.

\section{Intra- and interobserver variability}

The intra- and interobserver variability for all methods were moderate to excellent (for Aldrich 0.95 and 0.75 , for BARI 0.89 and 0.86, for APPROACH 0.89 and 0.79, for T2W-CMR 0.92 and 0.96 , for IS by DE 0.91 and 0.96 , for ESA by DE 0.90 and 0.87 , respectively).

Table 2. Number of patients (\%) with AAR greater than or equal to infarct size.

\begin{tabular}{lllll}
\hline & \multicolumn{3}{c}{ Subgroup } \\
\cline { 2 - 5 } & Overall & Subendocardial & Intermediate & Near transmural \\
\hline Aldrich (\%) & $51(65)$ & $13(100)$ & $29(67)$ & $9(41)$ \\
BARI (\%) & $68(87)$ & $13(100)$ & $41(95)$ & $14(64)$ \\
\hline APPROACH (\%) & $59(76)$ & $13(100)$ & $35(81)$ & $11(50)$ \\
T2W (\%) & $68(87)$ & $13(100)$ & $36(84)$ & $19(86)$ \\
ESA (\%) & $76(97)$ & $13(100)$ & $43(100)$ & $20(91)$ \\
\hline
\end{tabular}

Abbreviations: $\mathrm{AAR}=$ area at risk; $\mathrm{T} 2 \mathrm{~W}=\mathrm{T} 2$-weighted cardiovascular magnetic resonance; $\mathrm{ESA}=$ infarct endocardial surface area.

\section{AAR greater or equal to IS}

Table 2 shows that overall, 51 (65\%), 68 (87\%), 59 (76\%), 68 (87\%), and 76 (97\%) of patients using Aldrich, BARI, APPROACH, T2W, and ESA, respectively, showed an AAR that was greater than or equal to IS. Thus, ESA performed best and Aldrich worst. T2W and BARI performed equally well and were given a similar score of 3 (Table 4). In the subgroup of subendocardial infarcts, AAR was greater than or equal to IS in all patients, irrespective of the method used. The results of the other subgroups are shown in Table 2 as well.

\section{AAR approximation of IS in near transmural infarcts}

Bland-Altman analysis was used to assess the agreement between AAR and IS in a sub-cohort of patients ( $n=22$ ) with near transmural infarcts (Figure 1). Because near transmurality was defined as $>75 \%$ infarction and not $100 \%$ (mean transmurality was $82 \pm 4 \%$ ), a small positive bias was expected. This was true for all methods except for Aldrich (-3.5), which was thus considered the worst technique. The $95 \%$ limits of agreement, going from worst to best, were -22.9 to $29.6(\Delta 52.6),-28.3$ to $21.3(\Delta 49.6),-16.9$ to $20.0(\Delta 36.8),-6.9$ to $16.6(\Delta 23.6)$ and -4.3 to $18.0(\Delta 22.4)$ for BARI, Aldrich, APPROACH, T2w and ESA, respectively. Accordingly, ESA received the highest score, followed by T2w, APPROACH, BARI and Aldrich (Table 4). 

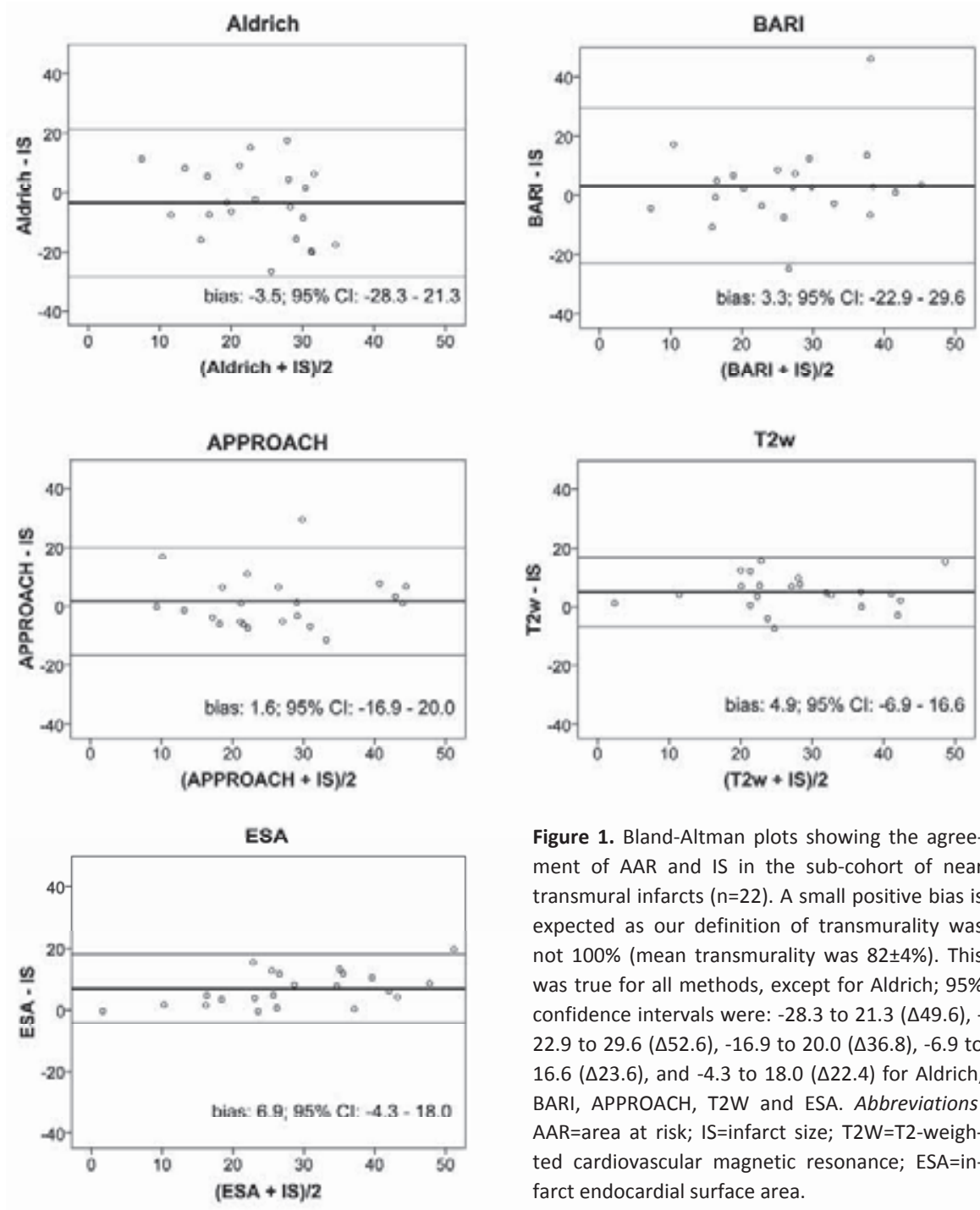

Figure 1. Bland-Altman plots showing the agreement of AAR and IS in the sub-cohort of near transmural infarcts $(n=22)$. A small positive bias is expected as our definition of transmurality was not $100 \%$ (mean transmurality was $82 \pm 4 \%$ ). This was true for all methods, except for Aldrich; $95 \%$ confidence intervals were: -28.3 to $21.3(\Delta 49.6)$, 22.9 to $29.6(\Delta 52.6),-16.9$ to $20.0(\Delta 36.8),-6.9$ to $16.6(\Delta 23.6)$, and -4.3 to $18.0(\Delta 22.4)$ for Aldrich, BARI, APPROACH, T2W and ESA. Abbreviations: $A A R=$ area at risk; IS=infarct size; $T 2 W=T 2$-weighted cardiovascular magnetic resonance; ESA=infarct endocardial surface area.

\section{Correlation of AAR and IS with respect to transmurality}

Figure 2 shows the correlation of AAR with IS, separated by transmurality subgroups. Physiology dictates that this correlation should improve with increasing infarct transmurality. For the Aldrich method, a reverse pattern was observed. For the BARI method, although the correlation went up from subendocardial to intermediate transmurality infarcts, it went back down in near transmural infarcts. For the remaining techniques, the correlations increased with increasing amount of 
transmurality. The correlation in near transmural infarcts was highest for ESA $(r=0.93, p \leq 0.001)$. Accordingly, ESA was given the highest relative score, followed by T2W, APPROACH, BARI, and Aldrich (Table 4).

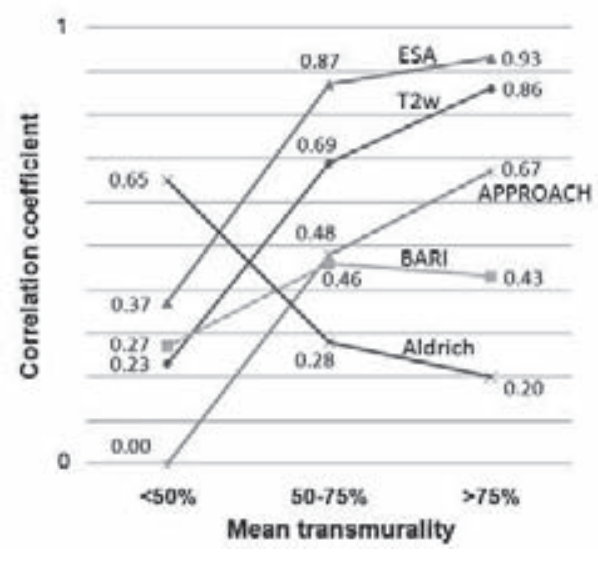

Figure 2. Correlation of AAR with IS for each transmurality sub-cohort. According to physiologic rules, this correlation should increase with increasing infarct transmurality. This was observed for APPROACH, T2W and ESA and in the near transmural sub-cohort, the correlation was highest for ESA $(0.93(p<0.001)$. Abbreviations: $E S A=i n f a r c t$ endocardial surface area; $\mathrm{T} 2 \mathrm{~W}=\mathrm{T} 2-$ weighted cardiovascular magnetic resonance.

\section{Inverse relationship of myocardial salvage with transmurality (Figure 3)}

The relationship of myocardial salvage with infarct transmurality is expected to be inverse (i.e. myocardial salvage is large in subendocardial infarcts and minimal in near transmural infarcts), and ideally, observations should be close to the inverse 'line of identity' (defined as $100 \%$ minus transmurality). The percentage of patients within a $\pm 30 \%$ margin around the inverse 'line of identity' (high=better) were $56 \%$, $76 \%, 65 \%, 77 \%$ and $92 \%$ for Aldrich, BARI, APPROACH, T2W and ESA, respectively (Table 3). The SEE values were 64.7, 44.8, 33.1, 26.6 and 21.7 for Aldrich, BARI, APPROACH, T2W and ESA, respectively. Based on the latter, the ESA method was given the highest score and the Aldrich method the lowest (Table 4). 

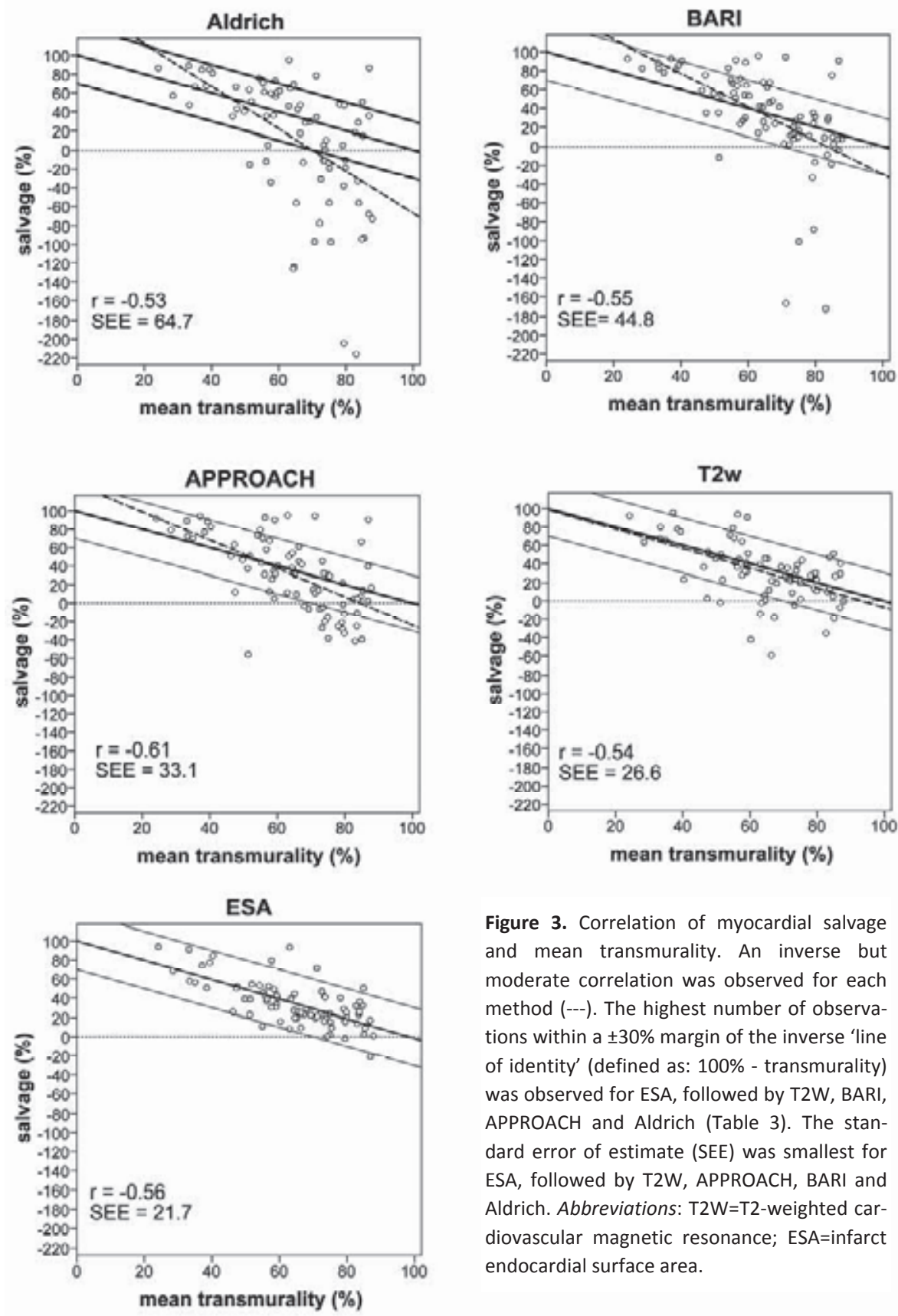

Figure 3. Correlation of myocardial salvage and mean transmurality. An inverse but moderate correlation was observed for each method (---). The highest number of observations within a $\pm 30 \%$ margin of the inverse 'line of identity' (defined as: 100\% - transmurality) was observed for ESA, followed by T2W, BARI, APPROACH and Aldrich (Table 3). The standard error of estimate (SEE) was smallest for ESA, followed by T2W, APPROACH, BARI and Aldrich. Abbreviations: $\mathrm{T} 2 \mathrm{~W}=\mathrm{T} 2$-weighted cardiovascular magnetic resonance; ESA=infarct endocardial surface area. 
Table 3. Number of patients (\%) within different margins of the 'inverse line of identity'*.

\begin{tabular}{llll}
\hline & \multicolumn{3}{c}{ Margin } \\
\cline { 2 - 4 } & $\pm 10 \%(\mathrm{n}=78)$ & $\pm 20 \%(\mathrm{n}=78)$ & $\pm 30 \%(\mathrm{n}=78)$ \\
\hline Aldrich (\%) & $13(17)$ & $32(41)$ & $44(56)$ \\
BARI (\%) & $18(23)$ & $49(63)$ & $59(76)$ \\
APPROACH (\%) & $20(26)$ & $38(49)$ & $51(65)$ \\
T2W (\%) & $33(42)$ & $53(68)$ & $60(77)$ \\
ESA (\%) & $38(49)$ & $62(79)$ & $72(92)$ \\
\hline
\end{tabular}

*Inverse line of identity: [(AAR - IS)/AAR] $\times 100$ ]. Margins of $\pm 10 \%, \pm 20 \%$ and $\pm 30 \%$ were randomly chosen to indicate the proximity to the 'inverse line of identity'. With respect to the $\pm 30 \%$ margin, ranking from best to worst is as follows: ESA, T2W, BARI, and APPROACH. Abbreviations: T2W=T2weighted cardiovascular magnetic resonance; $E S A=$ infarct endocardial surface area.

Table 4. Final scoring.

\begin{tabular}{|c|c|c|c|c|c|}
\hline \multirow[b]{2}{*}{ Method } & \multicolumn{4}{|c|}{ Physiologic concept } & \multirow[b]{2}{*}{ Final score } \\
\hline & $A A R \geq I S$ & $A A R=I S *$ & $\begin{array}{l}\text { Correlation with } \\
\text { transmurality }\end{array}$ & $\begin{array}{l}\text { Line of identity } \\
\mathrm{fit}^{+}\end{array}$ & \\
\hline Aldrich & 1 & 1 & 1 & 1 & 4 \\
\hline BARI & 3 & 2 & 2 & 2 & 9 \\
\hline APPROACH & 2 & 3 & 3 & 3 & 11 \\
\hline $\mathrm{T} 2 \mathrm{~W}$ & 3 & 4 & 4 & 4 & 15 \\
\hline ESA & 5 & 5 & 5 & 5 & 20 \\
\hline
\end{tabular}

Each AAR method was ranked and given a relative score (1 (worst) to 5 (best)), according to how well they agreed with specified physiologic concepts. The last column shows the final scores after summation. The ESA method performed best, followed by T2W, APPROACH, BARI and Aldrich. * In near transmural infarcts; ${ }^{\dagger}$ (salvage=100\%-transmurality). Abbreviations: $\mathrm{AAR}=$ area at risk; IS=infarct size; $\mathrm{T} 2 \mathrm{~W}=\mathrm{T} 2$ weighted cardiovascular magnetic resonance; ESA=infarct endocardial surface area.

\section{Final scores}

Table 4 shows the final scores assigned to each AAR method according to how well they agreed with the specified physiologic concepts. From worst to best, the methods were as follows: Aldrich, BARI, APPROACH, T2W-CMR and ESA.

\section{Discussion}

The findings of our study can be summarized as follows. Using established physiologic concepts to test the performance of different AAR methods in STEMI patients, CMR methods were better than angiographic methods (BARI and AP- 
PROACH), which were better than electrocardiographic (Aldrich). Concerning CMR, the ESA method performed consistently better than the hyperintense area on $\mathrm{T} 2 \mathrm{~W}$ CMR.

There is a major interest in measuring the AAR and myocardial salvage, which can be used as surrogate endpoints in clinical trials evaluating the effectiveness of new treatment strategies in AMI. Although being an accepted technique for the clinical assessment of AAR, SPECT suffers several limitations, such as 24-hour isotope availability, the need for isotope injection before reperfusion, suboptimal spatial resolution, and radiation burden. ${ }^{7}$ To validate the effectiveness of other methods for measuring the AAR that are more clinically feasible, we used physiologic concepts that are based on the transmural "wavefront phenomenon" of infarct progression. In essence, animal models have shown that cell death in prolonged coronary occlusion progresses in a transmural wave front over a period of 3-6 hours, and that the subendocardial lateral boundaries of the infarct are established within the first 40 minutes. ${ }^{2}$ Because DE-CMR accurately distinguishes between viable and nonviable myocardium, ${ }^{4,5}$ and has the potential to detect even small subendocardial infarcts, ${ }^{23}$ it may be useful as a clinical reference standard for the relative comparison of methods that measure the AAR. Scoring systems based on the electrocardiogram and coronary angiogram are attractive since these techniques are routinely performed in the acute phase of STEMI and are universally available.

The ST segment deviation score developed by Aldrich et al. is based on the number of leads with, and extent of, initial ST segment deviation. This AAR score was calibrated to predict final IS estimated by the Selvester QRS score in a population that did not receive reperfusion therapy. ${ }^{8}$ Our results show that the Aldrich score performed poor in estimating the AAR and confirm the result of a previous study using SPECT. ${ }^{24}$ The poor performance of the ECG method might be explained by the fact that the ECG only momentarily captures the ST segment, which is known to behave dynamically during the acute phase of STEMI. In addition, the ST segment changes that are prominent during coronary occlusion represent the ischemic myocardium, which will resolve when the ischemic myocardium is either infarcted or reperfused. With progressive myocardial necrosis, the ST segment deviation that is initially directing toward the ischemic region will be replaced by QRS deviation away from the infarcted region. ${ }^{25,26}$ The Aldrich score will therefore progressively underestimate the initial AAR when infarction proceeds.

The angiographic BARI and APPROACH myocardial jeopardy scores have shown to provide independent prognostic information in patients with chronic ischemic heart disease. ${ }^{10}$ In these methods, the jeopardized myocardium is based on the territory supplied by all significantly stenotic vessels ( $\geq 70 \%$ for the APPROACH and $>50 \%$ for the BARI scoring system). Of interest, neither angiographic method has been validated with a proper reference standard, i.e. SPECT, nor for its use to quan- 
tify the AAR in STEMI patients. Moreover, a previous investigation evaluating 5 other angiographic estimates of the AAR (not including BARI and APPROACH) showed a poor correlation with the AAR as defined by technetium-99m sestamibi imaging, suggesting a limited value of the coronary angiogram to measure the AAR. ${ }^{27}$ Of note, the extent of collateral flow is a major determinant of AAR that is not taken into account in any of the angiographic scoring systems. ${ }^{28}$ Recently, OrtizPérez et al. found an excellent correlation between both BARI and APPROACH and IS in the subgroup of patients with near transmural infarcts, suggesting indirectly that these techniques might be useful to measure the AAR ( $r=0.90, p<0.001$ for both). ${ }^{15}$ Despite the fact that our definition for near transmurality was more strict (>75\% vs. $>50 \%$ for Ortiz-Perez et al.), our correlations for BARI and APPROACH were only moderate at best ( $r=0.43$ and $r=0.67$, respectively) and thus we could not confirm their results. In addition, our results showed that up to $50 \%$ of patients (for APPROACH) had an AAR that was smaller than IS in the sub-cohort of near transmural infarcts.

An increasing number of studies are reporting on the assessment of the AAR by CMR, using dark-blood T2W-CMR sequences that are widely available on current scanners. ${ }^{29,} 30$ Although promising, dark-blood T2W-CMR suffers several technical limitations ${ }^{31}$ and validation and reproducibility are still limited. ${ }^{12,13,16}$ Low-signalto-noise-ratios between normal and injured myocardium, artifacts, such as inferolateral wall signal loss due to cardiac motion and bright subendocardial rims due to stagnant blood either may under- or overestimate the AAR. Furthermore, the signal intensity thresholds used to define abnormal myocardium for both DE- and T2WCMR are arbitrary, which could clearly affect IS, AAR and salvage calculations. So far, the results of studies using dark-blood T2W-CMR for measuring the AAR are confusing. While some studies have reported that the AAR $\geq I S$ in almost $100 \%$ of patients, we and others have observed this in only $87 \%$ and $65 \% .{ }^{32}$ Newer methods, such as bright blood T2-prepared steady state free precession (SSFP), hybrid methods between TSE and SSFP and contrast-enhanced SSFP-cine-imaging are promising and may improve accuracy. ${ }^{33,34,35}$

In a recent small study (38 STEMI patients), Berry et al. also compared electrocardiographic (but not Aldrich) and angiographic methods (APPROACH and the Duke jeopardy score) with T2W-CMR imaging to assess the AAR. ${ }^{29}$ They found a good correlation ( $r=0.78, p<0.0001)$ and agreement ( $2.5 \%$ bias on Bland-Altman analysis) between T2W-CMR and the APPROACH method for measuring the AAR. This finding led to their conclusion that T2W-CMR is a good measure for the AAR. In their study they only used one physiological concept to check for internal consistency, which was that $A A R \geq I S$. For both T2W-CMR and APPROACH, higher percentages of patients with $A A R \geq I S$ were found than in our study ( $92 \%$ and $88 \%$ vs. $87 \%$ and $76 \%$, respectively). They did not test the other complementary physiologic concepts as was performed in our study. Based on our findings it does not seem appropriate to 
validate T2W-CMR against the angiographic techniques (i.e. APPROACH) since 1) we did not find a good relationship between T2W and angiographic measures of AAR and 2) APPROACH performed poorly against our reference standard.

The ESA method is a relatively new but promising method to assess the AAR, which is measured on DE-CMR images. ${ }^{15}$ It is based on the transmural wave front progression of necrosis, and the setting of the lateral boundaries of the AAR by the lateral boundaries of infarction after 40 minutes. ${ }^{2}$ Variation in the percent of the AAR that becomes necrotic is primarily caused by the variation in transmural extent of infarction. ${ }^{21}$ Measuring the infarct ESA as a percentage of total LV subendocardial surface area would correspond to the AAR. Of all tested methods for measuring the AAR, we found that infarct ESA consistently performed best. Further validation is needed, because the results of two other studies have been conflicting. Although the AAR by the ESA method was smaller than by T2W-CMR in both studies, the correlation between them was good $\left(r=0.77(p<0.001)\right.$ in one study, ${ }^{14}$ but was low $(r=0.41, p=0.002)$ in the other study. ${ }^{36}$ Whether this discrepancy is caused by variation in either the ESA or T2W-CMR measurements remains unresolved.

For the determination of myocardial salvage, the use of different techniques to assess the individual components of AAR and IS may cause an offset problem. In other words, two different techniques may be highly correlated, but one may always be consistently under- or overestimating the other. Therefore the combined use appears problematic, especially in combining electrocardiographic and angiographic measurements with CMR techniques. For instance if the AAR is smaller that IS, the measurement of salvage will be useless.

\section{Limitations}

Our results are based on a limited number of patients and should therefore be interpreted with care. However this is a larger study so far to compare different techniques for measuring the AAR against a reference standard. We did not have SPECT imaging available to provide precise measurements of the AAR, but based on our physiologic concepts we are confident that our conclusions are valid. For example, when using the APPROACH method, the AAR was smaller than IS by DE-CMR in $24 \%$ of patients overall and the correlation with near transmural infarcts was only moderate, indicating this method is poor. We used specific cutoffs to quantify the AAR and IS by $T 2 w$ and DE-CMR ( $>5$ and $>2$ SD), which was based on the literature. There are certain limitations as we have discussed already with these cutoffs and although this may affect absolute size measurements, the correlation between methods remains unchanged. 


\section{Conclusion}

For measuring AAR, CMR methods performed better than angiographic methods, which performed better than electrocardiographic. Concerning CMR, ESA performed consistently performed better than T2W. Our results also show that combining electrocardiographic or angiographic methods of AAR with CMR determined infarct size, the determination of salvage may be problematic. 


\section{References}

1. An international randomized trial comparing four thrombolytic strategies for acute myocardial infarction. The GUSTO investigators. N Engl J Med. 1993;329(10):673-682.

2. Reimer KA, Lowe JE, Rasmussen MM, Jennings RB. The wavefront phenomenon of ischemic cell death. 1. Myocardial infarct size vs duration of coronary occlusion in dogs. Circulation. 1977;56(5):786-794.

3. Christian TF, Schwartz RS, Gibbons RJ. Determinants of infarct size in reperfusion therapy for acute myocardial infarction. Circulation. 1992;86(1):81-90.

4. Kim RJ, Fieno DS, Parrish TB, Harris K, Chen EL, Simonetti O, Bundy J, Finn JP, Klocke FJ, Judd RM. Relationship of MRI delayed contrast enhancement to irreversible injury, infarct age, and contractile function. Circulation. 1999;100(19):1992-2002.

5. Fieno DS, Kim RJ, Chen EL, Lomasney JW, Klocke FJ, Judd RM. Contrast-enhanced magnetic resonance imaging of myocardium at risk: distinction between reversible and irreversible injury throughout infarct healing. J Am Coll Cardiol. 2000;36(6):1985-1991.

6. Sinusas AJ, Trautman KA, Bergin JD, Watson DD, Ruiz M, Smith WH, Beller GA. Quantification of area at risk during coronary occlusion and degree of myocardial salvage after reperfusion with technetium-99m methoxyisobutyl isonitrile. Circulation. 1990;82(4):1424-1437.

7. Gibbons RJ, Verani MS, Behrenbeck T, Pellikka PA, O'Connor MK, Mahmarian JJ, Chesebro JH, Wackers FJ. Feasibility of tomographic 99mTc-hexakis-2-methoxy-2-methylpropyl-isonitrile imaging for the assessment of myocardial area at risk and the effect of treatment in acute myocardial infarction. Circulation. 1989;80(5):1277-1286.

8. Aldrich HR, Wagner NB, Boswick J, Corsa AT, Jones MG, Grande P, Lee KL, Wagner GS. Use of initial ST-segment deviation for prediction of final electrocardiographic size of acute myocardial infarcts. Am J Cardiol. 1988;61(10):749-753.

9. Alderman EL, Stadius, M. The angiographic definitions of the Bypass Angioplasty Revascularization Investigation. Coronary Artery Disease. 1992;3(12):1189-1207.

10. Graham MM, Faris PD, Ghali WA, Galbraith PD, Norris CM, Badry JT, Mitchell LB, Curtis MJ, Knudtson ML. Validation of three myocardial jeopardy scores in a population-based cardiac catheterization cohort. Am Heart J. 2001;142(2):254-261.

11. Jennings RB. Effect of reperfusion late in the phase of reversible ischemic injury changes in cell volume, electrolytes, metabolites, and infrastructure. Circulation research. 1985(56):262-278.

12. Aletras AH, Tilak GS, Natanzon A, Hsu LY, Gonzalez FM, Hoyt RF, Jr., Arai AE. Retrospective determination of the area at risk for reperfused acute myocardial infarction with T2-weighted cardiac magnetic resonance imaging: histopathological and displacement encoding with stimulated echoes (DENSE) functional validations. Circulation. 2006;113(15):1865-1870.

13. Tilak GS, Hsu LY, Hoyt RF, Jr., Arai AE, Aletras AH. In vivo T2-weighted magnetic resonance imaging can accurately determine the ischemic area at risk for 2-day-old nonreperfused myocardial infarction. Invest Radiol. 2008;43(1):7-15.

14. Wright J, Adriaenssens T, Dymarkowski S, Desmet W, Bogaert J. Quantification of myocardial area at risk with T2-weighted CMR: comparison with contrast-enhanced CMR and coronary angiography. JACC Cardiovasc Imaging. 2009;2(7):825-831.

15. Ortiz Perez JT, Meyers SN, Lee DC, Kansal P, Klocke FJ, Holly TA, Davidson CJ, Bonow RO, Wu E. Angiographic estimates of myocardium at risk during acute myocardial infarction: validation study using cardiac magnetic resonance imaging. Eur Heart J. 2007;28(14):1750-1758.

16. Carlsson M, Ubachs JF, Hedstrom E, Heiberg E, Jovinge S, Arheden H. Myocardium at risk after acute infarction in humans on cardiac magnetic resonance: quantitative assessment during follow-up and validation with single-photon emission computed tomography. JACC CardiovasC Imaging. 2009;2(5):569-576. 
17. Friedrich MG, Abdel Aty H, Taylor A, Schulz Menger J, Messroghli D, Dietz R. The salvaged area at risk in reperfused acute myocardial infarction as visualized by cardiovascular magnetic resonance. $J$ Am Coll Cardiol. 2008;51(16):1581-1587.

18. Thygesen K, Alpert JS, White HD, Jaffe AS, Apple FS, Galvani M, Katus HA, Newby LK, Ravkilde J, Chaitman B, Clemmensen PM, Dellborg M, Hod H, Porela P, Underwood R, Bax JJ, Beller GA, Bonow R, Van der Wall EE, Bassand JP, Wijns W, Ferguson TB, Steg PG, Uretsky BF, Williams DO, Armstrong PW, Antman EM, Fox KA, Hamm CW, Ohman EM, Simoons ML, Poole-Wilson PA, Gurfinkel EP, Lopez-Sendon JL, Pais P, Mendis S, Zhu JR, Wallentin LC, Fernandez-Aviles F, Fox KM, Parkhomenko AN, Priori SG, Tendera M, Voipio-Pulkki LM, Vahanian A, Camm AJ, De Caterina R, Dean V, Dickstein K, Filippatos G, Funck-Brentano C, Hellemans I, Kristensen SD, McGregor K, Sechtem U, Silber S, Widimsky P, Zamorano JL, Morais J, Brener S, Harrington R, Morrow D, Lim M, Martinez-Rios MA, Steinhubl S, Levine GN, Gibler WB, Goff D, Tubaro M, Dudek D, Al-Attar N. Universal definition of myocardial infarction. Circulation. 2007;116(22):2634-2653.

19. Rentrop KP, Cohen M, Blanke H, Phillips RA. Changes in collateral channel filling immediately after controlled coronary artery occlusion by an angioplasty balloon in human subjects. J Am Coll Cardiol. 1985;5(3):587-592.

20. The Thrombolysis in Myocardial Infarction (TIMI) trial. Phase I findings. TIMI Study Group. N Engl J Med. 1985;312(14):932-936.

21. Lee JT, Ideker RE, Reimer KA. Myocardial infarct size and location in relation to the coronary vascular bed at risk in man. Circulation. 1981;64(3):526-534.

22. Bondarenko O, Beek AM, Hofman MB, Kuhl HP, Twisk JW, van Dockum WG, Visser CA, van Rossum AC. Standardizing the definition of hyperenhancement in the quantitative assessment of infarct size and myocardial viability using delayed contrast-enhanced CMR. J Cardiovasc Magn Reson. 2005;7(2):481-485.

23. Wagner A, Mahrholdt H, Holly TA, Elliott MD, Regenfus M, Parker M, Klocke FJ, Bonow RO, Kim RJ, Judd RM. Contrast-enhanced MRI and routine single photon emission computed tomography (SPECT) perfusion imaging for detection of subendocardial myocardial infarcts: an imaging study. Lancet. 2003;361(9355):374-379.

24. Christian TF, Gibbons RJ, Clements IP, Berger PB, Selvester RH, Wagner GS. Estimates of myocardium at risk and collateral flow in acute myocardial infarction using electrocardiographic indexes with comparison to radionuclide and angiographic measures. J Am Coll Cardiol. 1995;26(2):388-393.

25. Sederholm M, Erhardt L, Sjogren A. Continuous vectorcardiography in acute myocardial infarction. Natural course of ST and QRS vectors. Int J Cardiol. 1983;4(1):53-63.

26. Anderson $\mathrm{Cl}$, Harrison DG, Stack NC, Hindman NB, Ideker RE, Palmeri ST, Selvester RH, Wagner GS. Evaluation of serial QRS changes during acute inferior myocardial infarction using a QRS scoring system. Am J Cardiol. 1983;52(3):252-256.

27. Haronian HL, Remetz MS, Sinusas AJ, Baron JM, Miller HI, Cleman MW, Zaret BL, Wackers FJ. Myocardial risk area defined by technetium-99m sestamibi imaging during percutaneous transluminal coronary angioplasty: comparison with coronary angiography. J Am Coll Cardiol. 1993;22(4):10331043.

28. Klarich KW, Christian TF, Higano ST, Gibbons RJ. Variability of myocardium at risk for acute myocardial infarction. Am J Cardiol. 1999;83(8):1191-1195.

29. Berry C, Kellman P, Mancini C, Chen MY, Bandettini WP, Lowrey T, Hsu LY, Aletras AH, Arai AE. Magnetic resonance imaging delineates the ischemic area at risk and myocardial salvage in patients with acute myocardial infarction. Circ Cardiovasc Imaging. 2010 Sep 1;3(5):527-535.

30. Eitel I, Desch S, Fuernau G, Hildebrand L, Gutberlet M, Schuler G, Thiele H. Prognostic significance and determinants of myocardial salvage assessed by cardiovascular magnetic resonance in acute reperfused myocardial infarction. J Am Coll Cardiol. 2010 Jun 1;55(22):2470-9.

31. Wince WB, Kim RJ. Molecular imaging: T2-weighted CMR of the area at risk--a risky business? Nat Rev Cardiol. 2010 Oct 7(10):547-549. 
32. Payne AR, Casey M, McClure J, McGeoch R, Murphy A, Woodward R, Saul A, Bi X, Zuehlsdorff S, Oldroyd KG, Tzemos N, Berry C. Bright Blood T2 Weighted MRI Has Higher Diagnostic Accuracy Than Dark Blood STIR MRI for Detection of Acute Myocardial Infarction and for Assessment of the Ischemic Area-at-Risk and Myocardial Salvage. Circ Cardiovasc Imaging. 2011 Mar 22.

33. Aletras AH, Kellman P, Derbyshire JA, Arai AE. ACUT2E TSE-SSFP: a hybrid method for T2-weighted imaging of edema in the heart. Magn Reson Med. 2008 Feb;59(2):229-35

34. Kellman P, Aletras AH, Mancini C, McVeigh ER, Arai AE. T2-prepared SSFP improves diagnostic confidence in edema imaging in acute myocardial infarction compared to turbo spin echo. Magn Reson Med. 2007 May;57(5):891-7.

35. Sörensson P, Heiberg E, Saleh N, Bouvier F, Caidahl K, Tornvall P, Rydén L, Pernow J, Arheden H. J Cardiovasc Magn Reson. 2010 Apr 30;12(1):25.Assessment of myocardium at risk with contrast enhanced steady-state free precession cine cardiovascular magnetic resonance compared to singlephoton emission computed tomography.

36. Ubachs JF, Engblom H, Erlinge D, Jovinge S, Hedström E, Carlsson M, Arheden H. Cardiovascular magnetic resonance of the myocardium at risk in acute reperfused myocardial infarction: comparison of T2-weighted imaging versus the circumferential endocardial extent of late gadolinium enhancement with transmural projection. J Cardiovasc Magn Reson. 2010 Mar 29;12:18. 
CHAPTER 7

General discussion 
Chapter 7 discusses the results of this thesis in the light of existing evidence or controversy and future perspectives will be given.

\section{MVO: underlying pathophysiology and clinical diagnosis}

Coronary occlusion results in ischemia within the region of the coronary distribution, defined as the area at risk (AAR). Without restoration of blood flow, myocardial necrosis progresses as a 'wavefront' from the subendocardium toward the subepicardium. ${ }^{1}$ A complex relationship exists between myocyte necrosis and capillary damage, the latter resulting in the 'no-reflow' phenomenon or MVO. The underlying pathophysiological mechanisms of myocyte death and MVO are complex, multifactorial and not fully understood. Ischemia results in myocyte and vascular injury, and both can be aggravated by additional reperfusion injury. A balance exists between the benefits of reperfusion to reduce infarct size on the one hand and reperfusion injury on the other hand, depending on the duration of occlusion. If ischemic myocardium is reperfused early, infarct size is limited and additional reperfusion injury minimal. At the other extreme, when ischemic myocardium is reperfused late, necrosis will be near transmural and additional reperfusion injury will also be minimal. Thus, a relatively narrow time window exists, during which reperfusion injury may be preventable. ${ }^{2}$

Ischemia induces the accumulation of intracellular sodium, hydrogen and calcium ions in myocytes as well as in endothelial cells. ${ }^{3}$ Corresponding changes include endothelial cell swelling, the formation of intraluminal blebs, fibrin deposition and extravascular compression by surrounding edematous myocytes. ${ }^{4}$ Ambrosio et al., and later Reffelman et al., demonstrated a progressive expansion of microvascular damage during reperfusion, which is assumed to be the result of reperfusion injury. ${ }^{5,6}$ The precise pathophysiology of reperfusion injury remains to be established, but involves several complex molecular and cellular mechanisms. Reperfusion markedly enhances intracellular calcium loading, mitochrondrial permeability transition pore (MPTP) opening, infiltration of neutrophils and platelets into the ischemic region that adhere to endothelial cells, form aggregates and plug capillaries. ${ }^{4,7}$ Furthermore, neutrophil adhesion is followed by activation, diapedesis, and migration into surrounding tissue and release of oxygen free radicals, proteolytic enzymes, and pro-inflammatory mediators. ${ }^{8}$ This may then lead to additional myocyte and endothelial cell damage.

Reperfusion injury thus affects both myocytes as well as the endothelium. Whether this always evolves in parallel or whether progressive MVO during reperfusion can contribute to additional myocyte death is still controversial. ${ }^{9}$ An argument against this comes from a recent randomized, double blind, placebo-controlled trial, investigating the efficacy of the naturally occurring peptide FX06 to reduce infarct 
size. ${ }^{10}$ The results of this trial showed that, although MVO was more than halved in patients treated with FX06, no major difference in infarct size at 5 days and 4 months was found. Furthermore, animal studies have shown that the occurrence of microvascular damage is strongly related to the duration of ischemia prior to reperfusion and always lags behind myocardial cell injury. ${ }^{11,12}$ Whether this sequence of events is similar during reperfusion is unknown, but if so, would make additional myocyte death as a consequence of MVO less likely. Because infarct size has been shown to be the major determinant of $\mathrm{MVO}^{13}$, the correlation of MVO with clinical outcome might solely reflect the correlation of MVO with infarct size. ${ }^{14}$

After the first description of MVO in animal models, compromised reperfusion was also recognized clinically with a variable prevalence, ranging from $5 \%$ up to $50 \%$, depending on the methods used and population studied. ${ }^{15}$ In humans, PCIrelated distal embolization of atherothrombotic material significantly contributes to MVO. Although there are several quantitative measures of angiographic perfusion such as Thrombolysis In Myocardial Infarction (TIMI) flow grading, corrected TIMI frame count, TIMI myocardial perfusion grade and blush score, none of these techniques directly visualize the affected myocardium. ${ }^{16-18}$ Similarly, rapid resolution of initial ST-segment elevation (STR) following reperfusion therapy indicates full and prompt restoration of myocardial tissue perfusion, but remains a surrogate biomarker. ${ }^{19}$ In contrast, myocardial contrast echocardiography (MCE) and cardiovascular magnetic resonance imaging (CMR) are able to directly visualize and quantify MVO. ${ }^{20-24}$ Because CMR allows accurate and reproducible assessment of ventricular function, infarct size and MVO in one single examination as opposed to MCE, it is the preferred technique to assess the extent of injury after acute myocardial infarction (AMI).

\section{Detection and characteristics of MVO}

Investigators have used different CMR techniques for the detection of MVO, but there is no clear consensus about which technique is most accurate. First pass perfusion (FPP) CMR dynamically tracks the first pass of a contrast agent in the myocardium during contrast injection. MVO can be shown as a persistent subendocardial perfusion defect due to delayed wash-in of contrast in the infarct core. MVO using this technique was validated with thioflavin-S staining, one of the pathologic gold standards of detecting MVO. ${ }^{22}$ With traditional delayed enhancement (DE) CMR, imaging starts 10-15 minutes after contrast injection and MVO can be observed as a central hypointense region within the hyperenhanced (infarcted) myocardium. ${ }^{20}$ MVO detected either by FPP-CMR or DE-CMR has prognostic significance regarding LV remodeling and outcome. ${ }^{20,25-28}$ 
As gadolinium is freely permeable through the vascular wall and passively diffuses into the area of MVO within 10-15 minutes, FPP-CMR may be more sensitive than DE-CMR for the detection of MVO. On the other hand, FPP-CMR has a lower spatial resolution, signal-to-noise ratio and incomplete LV coverage as compared to DE-CMR. In chapter 3, we used an optimized single breathhold 3D inversion recovery gradient echo technique (IR-GRE) for imaging MVO early and late after contrast injection. With this technique we combined the advantage of imaging MVO early after contrast injection with the higher spatial resolution and complete LV coverage of a regular DE-CMR sequence. With this approach, we found a higher prevalence of early MVO than late MVO (63\% vs. $54 \%, p=0.008)$. In addition, we showed that patients with early MVO but not late MVO (MVO disappearance) had infarct sizes and LV ejection fractions (LVEF) that were intermediate between patients with persistent (late) MVO and without any MVO.

There are only few comparisons in the literature between FPP-CMR and DECMR for the detection of MVO. In the study by Lund et al., FPP-CMR depicted MVO in $38 \%$ of 60 patients, whereas MVO was observed on DE-CMR in only $28 \%$ of patients. ${ }^{29}$ A limitation of this study was the incomplete LV coverage by FPP-CMR (single slice) and DE-CMR (3 slices). Nijveldt et al. used FPP-CMR (early hypoenhancement), a 3D IR-GRE sequence run at 2 minutes (intermediate enhancement), and a regular 2D DE-CMR sequence run at 12-15 minutes after contrast injection (late hypoenhancement) to detect MVO. ${ }^{30}$ The prevalence of MVO on early, intermediate and late hypoenhancement imaging was $70 \%, 62 \%$ and $59 \%$, respectively, which is comparable to our findings. They also found that late hypoenhancement was the strongest predictor of LV volumes and LVEF at follow-up. They did not specifically investigate the long-term prognostic implications of intermediate hypoenhancement (MVO on early but not late imaging).

Microvascular flow following reperfusion is spatially and temporally complex with coexisting regions of hyperemia, impaired vasodilatory flow reserve, low flow and almost zero reflow. In addition, the spatial extent of these perfusion patterns may vary over time. ${ }^{5,31-33}$ Improvement in microvascular dysfunction may even occur early after myocardial infarction ( $<1$ week) and may be beneficial in preventing left ventricular remodeling. ${ }^{34}$ Our approach of using the same pulse sequence for early and late imaging - except for a longer inversion time - allowed us to study the dynamic changes of MVO over time going from early to late imaging, and occuring within minutes. We demonstrated that the decrease of MVO even in this short time period is heterogeneous, which might reflect differences in underlying microvascular damage. The decrease and disappearance of early MVO during late imaging was only related to the initial size of MVO, but not to other clinical variables. Whether this aspect has clinical implications is currently unknown, but could be helpful in evaluating therapies that limit reperfusion injury. We recommend that both early and late MVO imaging is performed in the current evaluation of AMI by 
CMR. Our optimized single breathhold 3D IR-GRE technique is fast, accurate and can be easily implemented in current clinical and study protocols.

\section{Microvascular obstruction and intramyocardial hemorrhage}

MVO is a consequence of ischemia and reperfusion and may lead to intramyocardial hemorrhage (IMH) in the infarct core by extravasation of red blood cells through damaged endothelium. ${ }^{35-37} \mathrm{IMH}$ has been observed both after pharmacological reperfusion and mechanical reperfusion. ${ }^{38,} 39$ Inflammation in AMI increases local myocardial water content and increases signal intensity (SI) on T2 weighted imaging (T2W-CMR). On T2W-CMR, IMH can be observed as a central dark region within a hyperintense region and is caused by shortening of T2-relaxation due to the paramagnetic properties of predominantly deoxyhemoglobin. ${ }^{40}$ Although validation is still limited, these hypointense regions on T2W-CMR seem to correspond to the histological evidence of IMH. ${ }^{41}$ Thus, with CMR it is now possible to study these histological aspects of injury in AMI in vivo and in a single investigation and noninvasively. Previous studies have suggested that MVO, as detected by CMR, is independently related to adverse remodeling and clinical outcome. ${ }^{20,26,33}$ The clinical significance of IMH is less evident.

The results of our study in chapter 4 show that IMH and MVO are strongly related and that infarct size was the only independent predictor of LV remodeling. In contrast to our results and those of Beek et al. ${ }^{42}$, two recent other studies suggested that IMH is an independent predictor of adverse LV remodeling regardless of infarct size and MVO. ${ }^{43,44}$ These discrepancies could be explained by differences in definition and quantification of IMH. In contrast to the study by Ganame et al., who defined IMH as hypointense areas with mean SI of 2 standard deviations (SD) below the $\mathrm{SI}$ of the hyperintense peripheral region, we manually delineated regions of $\mathrm{IMH}$. In addition, a minimal volume of $1 \mathrm{~mL}$ for $\mathrm{IMH}$ was required for the definition in the study by Ganame et al. This may explain the lower prevalence of IMH in their study as compared to our study (24\% vs. $43 \%)$, suggesting either that more severe forms of IMH were studied and more subtle forms of IMH were excluded. As a result of this, the independent role of severe forms of $\mathrm{IMH}$ on adverse remodeling might have become more prominent.

Myocyte necrosis, MVO and IMH in AMI are strongly related, time-sensitive phenomena and the real mechanisms underlying these features are not yet well understood in humans. Both MVO and IMH depend on the duration of coronary occlusion but lag behind myocyte death. ${ }^{11,12}$ Similar to myocardial necrosis, both progress from the endocardium toward the epicardium as a 'wavefront'. After varying periods of ischemia, sequential changes of the microvasculature occur, resulting in MVO and loss of capillary integrity. Ensuing reperfusion then leads to extravasa- 
tion of erythrocytes from an already severely compromised microvasculature, resulting in IMH. ${ }^{35,36}$ We have shown that some patients with MVO did not have IMH and that infarct size and LVEF were in between average values of patients who had both $\mathrm{MVO}$ and $\mathrm{IMH}$, and patients without either $\mathrm{MVO}$ or $\mathrm{IMH}$. In line with these experimental studies, this suggests that the co-existence of $\mathrm{IMH}$ and $\mathrm{MVO}$ are a gradation of the underlying severity of ischemic injury. Further studies are necessary to clarify the clinical role of IMH beyond MVO or that it is just a marker of the already severely damaged microvasculature.

\section{ST segment recovery in relation to underlying infarct characteristics}

To decide whether patients may need adjunctive infarct size limiting therapies after optimal epicardial flow has been established, the determination of early STR is of great interest. Despite the general acceptance that early STR after successful epicardial flow establishment reflects myocardial reperfusion ${ }^{19,45}$, only a limited number of studies has directly related incomplete STR to microvascular dysfunction. ${ }^{24,46}$ Instead, incomplete STR has been predominantly investigated in relation to decreased myocardial salvage, larger infarct size, low LVEF, and adverse outcome. ${ }^{\text {47-50 }}$ Studies investigating the relation of STR with myocardial blush grading (MBG) have shown that still $35 \%$ of patients with MBG 3 have incomplete STR ${ }^{18}$. This suggests that STR is rather a direct reflection of injury to myocytes than of MVO, because the microvasculature in these patients is 'patent'.

Whether MVO can contribute to additional myocyte death during reperfusion, or that both evolve in parallel remains unresolved. MVO and infarct size are strongly related and CMR studies investigating their independent relationship with adverse remodeling have been inconsistent, with some favoring $\mathrm{MVO}^{20,30,51,52}$ and others favoring infarct size. ${ }^{23,53-55}$ This then hinders the clarification of the relationship of STR with adverse remodeling in relation to these underlying infarct characteristics. In chapter $\mathbf{5}$ we investigated the value of STR 30 and 60 minutes after mechanical reperfusion in relation to underlying infarct characteristics and adverse remodeling. Instead of evaluating the relationship of STR with these individual infarct characteristics, we used their correlational information and clustered patients into three different CMR groups at risk for adverse remodeling. Patients were clustered into a low, intermediate, and high CMR risk group, which was based on infarct size, transmurality, and MVO. By doing so, we first found that infarct size was more predictive for adverse remodeling than MVO and infarct transmurality. Second, and after evaluating multiple STR methods, we found that the most simple method of worst lead ST deviation (STD) determined as early as 30 minutes after reperfusion, accurately discriminated between the high and low CMR risk group. 
Our study is one of only few that have investigated STR in relation to CMR based characteristics of reperfused AMI and adverse remodeling during follow-up. Our results are different from those of Nijveldt et al., who found that residual ST segment elevation on the 12-lead ECG 60 minutes after reperfusion, was the strongest predictor of low initial LVEF and the presence of MVO at $4 \pm 2$ days, even after controlling for infarct size. ${ }^{56}$ No follow-up data are reported in that study. In a recent randomized trial comparing primary $\mathrm{PCl}$ with combined proximal embolic protection and thrombus aspiration, no significant differences in complete STsegment resolution at $60 \mathrm{~min}$. (primary end point) and infarct size or LVEF at followup CMR were found. ${ }^{57,58}$ In a post hoc analysis, STR appeared to be a strong, independent predictor of LVEF and infarct size at follow-up. ${ }^{59}$ In that study, a relationship of STR with MVO was not studied. A post hoc analysis of another clinical trial, investigating the effect of an infarct size limiting agent (FX06) in adjunction to $\mathrm{PCl}$, demonstrated that 90 minute worst-lead residual STD was associated with both final infarct size and long-term left ventricular function at 4 months. Our study is the first to demonstrate that worst-lead residual STD, by far the simplest method for calculating ST-segment recovery, can be measured as early as 30 minutes after reperfusion to predict adverse remodeling, which was mainly related to infarct size.

Comparing different parameters (i.e. STR and MVO) that are assessed at different time points after reperfusion might be problematic because both behave dynamically over time. This may explain the inconsistent results between our study and that of Nijveldt et al. ${ }^{30}$ We suggest that when CMR is used to clarify the relation of STR with MVO in future studies, both early and late MVO imaging should be performed as soon as possible after reperfusion.

\section{The area at risk}

The region of acutely hypoperfused myocardium within a coronary distribution during coronary occlusion is defined as the AAR. ${ }^{60}$ Without restoration of blood flow the AAR will be subject to near transmural infarction. ${ }^{1}$ If the AAR could be reliably estimated in combination with infarct size, then the 'salvaged' myocardium could be assessed, allowing evaluation of the quality of reperfusion and effectiveness of reperfusion therapies. Infarct size is accurately measurable with DE-CMR ${ }^{61}$, ${ }^{62}$, but clinical determination of the AAR remains challenging as the reference standard (i.e. SPECT) is hampered by radiation exposure, low spatial resolution and difficult logistics requiring tracer injection before reperfusion.

Both electrographic and coronary angiographic measures have been used to measure the AAR. ${ }^{63-65}$ Experimental studies have suggested that the hyperintense area on T2W-CMR delineates the AAR in AMI. ${ }^{66}$ This was soon followed by multiple clinical studies using this technique for measuring both AAR and myocardial salvage 
in combination with DE-CMR. ${ }^{67-71} \mathrm{~A}$ novel CMR technique is infarct endocardial surface area - ESA - by DE-CMR ${ }^{65}$ as a proportion of the total LV endocardial surface area. This method is based on the concept that myocardial necrosis progresses from endocardium toward the epicardium with minimal lateral extension. ${ }^{1}$ Although promising, more validation of these CMR methods in humans against appropriate reference standards is necessary. Thus far, angiographic myocardial jeopardy scores have been used to validate these CMR methods for measuring the AAR in humans. Of interest, these angiographic scores were originally developed to provide independent prognostic information in patients with chronic ischemic heart disease. ${ }^{64}$ Moreover, these scores themselves have not been validated against a reference standard (i.e. SPECT), nor for their use to quantify the AAR in STEMI patients. The angiographic methods may thus not be sufficiently qualified to be used as an appropriate reference standard. Recent studies comparing the angiographic with CMR methods have reported inconsistent results. For example, Ortiz-Pérez et al. found excellent correlations between the angiographic methods and infarct ESA ( $r=0.90$ for BARI and $r=0.87$ for APPROACH), whereas in a study by Wright et al., the correlation for BARI with ESA was only moderate ( $r=0.42){ }^{65,71}$

In chapter 6 we used multiple established physiologic concepts to evaluate the performance of electrocardiographic, angiographic and CMR methods for measuring the AAR. These physiologic concepts were based on the 'wavefront phenomenon' of infarct progression. ${ }^{1}$ Our methodology was quite different from other studies that used only one of these physiologic concepts to validate the angiographic reference standard (i.e. BARI and APPROACH). Furthermore, our results were different. Ortiz Pérez et al. found excellent correlations between the angiographic scores and infarct size in the sub-cohort of near transmural infarcts $(r=0.90)$, whereas this correlation was only moderate at best in our study ( $r=0.43-0.67) .{ }^{65}$ Berry et al. found that $92 \%$ and $88 \%$ of patients using T2W-CMR and APPROACH had an AAR $\geq$ infarct size, while in our study this was $87 \%$ and $76 \% .{ }^{67}$ The conclusions of these studies that the T2W-CMR and APPROACH methods are accurate measures of the AAR thus seem premature.

We concluded that the CMR methods performed better than the angiographic methods, which performed better than electrocardiographic methods to measure the AAR. Of the CMR methods, ESA performed better than T2W-CMR. Based on our results, the angiographic methods, BARI and APPROACH, should in fact not be used to validate other methods that measure the AAR.

\section{Future perspectives}

Modern STEMI treatment has reduced 30-day mortality below $4 \%{ }^{72}$, making it less feasible to demonstrate the benefit of adjunctive cardioprotective therapies. For 
demonstrating this, very large clinical trials would be needed. Given that quantification of LVEF, ventricular volumes and infarct size by CMR is very accurate and highly reproducible, these parameters can provide useful surrogate end points. ${ }^{73}$ In addition, these parameters have been shown to provide important independent prognostic information. ${ }^{74,75}$ Thus, CMR will become increasingly important to evaluate the efficacy of adjunctive therapeutic strategies either in the form of pharmacological $^{76}$ and mechanical interventions ${ }^{77,78}$ or cellular-based therapies. ${ }^{79}$ Separating ischemic injury from additional reperfusion injury remains clinically challenging. Previous studies have shown that bursts of ventricular arrhythmias early after reperfusion in patients with TIMI 3 flow and $\geq 50 \%$ stable ST-segment recovery are associated with larger infarct sizes and lower LVEF as determined with SPECT. ${ }^{80,81}$ Whether this ECG biomarker may be useful as a real-time parameter of reperfusion injury and also its relation to CMR derived parameters needs to be studied more thoroughly. Another important question to address in the future is the determination of the true prognostic significance of MVO and IMH and their disputable role in infarct progression. Since DE-CMR allows the simultaneous assessment of both MVO and IS, this technique is useful to demonstrate whether interventions that specifically ameliorate MVO or IMH could reduce final infarct size and improve prognosis. In addition, future developments such as molecular imaging and targeted contrast agents are likely to improve our understanding of reperfusion injury and determinants of LV remodeling. Infarct size and scar heterogeneity as determined with $\mathrm{CMR}$ are increasingly being recognized as significant risk factors for ventricular arrhythmias and sudden cardiac death (SCD). Eventually, this will better identify patients at risk for SCD, as current guidelines for implantation of internal cardioverter defibrillators are predominantly based on LVEF and lack sufficient sensitivity and specificity. ${ }^{82}$ Finally, the prognostic importance of non-Q-wave unrecognized $\mathrm{MI}$ (UMI) as detected by DE-CMR has recently been shown. ${ }^{83}$ Given the increasing prevalence of diabetes mellitus worldwide, this may have important implications for individual patients and for public health care. Population studies to determine the prevalence of UMI in diabetes mellitus and evaluate if early diagnosis with appropriate treatment will alter prognosis, are needed. 


\section{Conclusions}

The studies from this thesis demonstrate that by using an optimized CMR technique, the prevalence of MVO early after contrast injection is higher than with traditional DE-CMR. The extent of MVO going from early to late imaging decreases and this decrement is heterogeneous. Patients with early but late MVO disappearance have an infarct size and remodeling parameters that are intermediate between patients without MVO and those with both early MVO and late MVO persistence. $\mathrm{IMH}$ and MVO are frequently observed after reperfused AMI and are strongly related phenomena. From two separate studies, one investigating the role of infarct size, MVO and IMH and the other investigating the role of infarct size, transmurality and MVO, we found that infarct size was the best predictor of adverse remodeling after 3 months. Worst lead residual STD, determined as early as 30 minutes after $\mathrm{PCl}$, accurately discriminated between a high and combined low/intermediate CMR risk group and was mainly related to infarct size. For measuring AAR, CMR methods were better than angiographic methods, which were better than electrocardiographic methods. ESA by DE-CMR appeared to be the best method for measuring $A A R$ in vivo. Overall, the results of this thesis show that CMR accurately detects different underlying characteristics of injury in reperfused AMI and allows investigating their relation with adverse remodeling. CMR has great potential to unravel the complex underlying pathophysiology of ischemia-reperfusion in AMI in humans that must lead to adjunctive infarct size limiting therapies. 


\section{References}

1. Reimer KA, Lowe JE, Rasmussen MM, et al. The wavefront phenomenon of ischemic cell death. 1. Myocardial infarct size vs duration of coronary occlusion in dogs. Circulation. Nov 1977;56(5):786794.

2. Gersh BJ, Stone GW, White HD, et al. Pharmacological facilitation of primary percutaneous coronary intervention for acute myocardial infarction: is the slope of the curve the shape of the future? Jama. Feb 23 2005;293(8):979-986.

3. Niccoli G, Burzotta F, Galiuto L, et al. Myocardial no-reflow in humans. J Am Coll Cardiol. Jul 21 2009;54(4):281-292.

4. Bekkers SC, Yazdani SK, Virmani R, et al. Microvascular obstruction: underlying pathophysiology and clinical diagnosis. J Am Coll Cardiol. Apr 20 2010;55(16):1649-1660.

5. Ambrosio G, Weisman HF, Mannisi JA, et al. Progressive impairment of regional myocardial perfusion after initial restoration of postischemic blood flow. Circulation. Dec 1989;80(6):1846-1861.

6. Reffelmann T, Kloner RA. Microvascular reperfusion injury: rapid expansion of anatomic no reflow during reperfusion in the rabbit. Am J Physiol Heart Circ Physiol. Sep 2002;283(3):H1099-1107.

7. Ambrosio G, Tritto I. Reperfusion injury: experimental evidence and clinical implications. Am Heart J. Aug 1999;138(2 Pt 2):S69-75.

8. Engler RL, Dahlgren MD, Morris DD, et al. Role of leukocytes in response to acute myocardial ischemia and reflow in dogs. Am J Physiol. Aug 1986;251(2 Pt 2):H314-323.

9. Monassier JP. Reperfusion injury in acute myocardial infarction. From bench to cath lab. Part I: Basic considerations. Arch Cardiovasc Dis. Jul-Aug 2008;101(7-8):491-500.

10. Atar D, Petzelbauer P, Schwitter J, et al. Effect of intravenous FX06 as an adjunct to primary percutaneous coronary intervention for acute ST-segment elevation myocardial infarction results of the F.I.R.E. (Efficacy of FX06 in the Prevention of Myocardial Reperfusion Injury) trial. J Am Coll Cardiol. Feb 24 2009;53(8):720-729.

11. Higginson LA, Beanlands DS, Nair RC, et al. The time course and characterization of myocardial hemorrhage after coronary reperfusion in the anesthetized dog. Circulation. May 1983;67(5):10241031.

12. Kloner RA, Ganote CE, Jennings RB. The "no-reflow" phenomenon after temporary coronary occlusion in the dog. J Clin Invest. Dec 1974;54(6):1496-1508.

13. Reffelmann T, Hale SL, Li G, et al. Relationship between no reflow and infarct size as influenced by the duration of ischemia and reperfusion. Am J Physiol Heart Circ Physiol. Feb 2002;282(2):H766772.

14. Reffelmann T, Kloner RA. The "no-reflow" phenomenon: basic science and clinical correlates. Heart. Feb 2002;87(2):162-168.

15. Rezkalla SH, Kloner RA. Coronary no-reflow phenomenon: from the experimental laboratory to the cardiac catheterization laboratory. Catheter Cardiovasc Interv. Dec 1 2008;72(7):950-957.

16. Gibson CM, Murphy SA, Rizzo MJ, et al. Relationship between TIMI frame count and clinical outcomes after thrombolytic administration. Thrombolysis In Myocardial Infarction (TIMI) Study Group. Circulation. Apr 20 1999;99(15):1945-1950.

17. Morishima I, Sone T, Okumura K, et al. Angiographic no-reflow phenomenon as a predictor of adverse long-term outcome in patients treated with percutaneous transluminal coronary angioplasty for first acute myocardial infarction. J Am Coll Cardiol. Oct 2000;36(4):1202-1209.

18. van 't Hof AW, Liem A, Suryapranata $H$, et al. Angiographic assessment of myocardial reperfusion in patients treated with primary angioplasty for acute myocardial infarction: myocardial blush grade. Zwolle Myocardial Infarction Study Group. Circulation. Jun 16 1998;97(23):2302-2306.

19. de Lemos JA, Braunwald E. ST segment resolution as a tool for assessing the efficacy of reperfusion therapy. J Am Coll Cardiol. Nov 1 2001;38(5):1283-1294. 
20. Hombach V, Grebe O, Merkle N, et al. Sequelae of acute myocardial infarction regarding cardiac structure and function and their prognostic significance as assessed by magnetic resonance imaging. Eur Heart J. Mar 2005;26(6):549-557.

21. Ito H, Okamura A, Iwakura K, et al. Myocardial perfusion patterns related to thrombolysis in myocardial infarction perfusion grades after coronary angioplasty in patients with acute anterior wall myocardial infarction. Circulation. Jun 1 1996;93(11):1993-1999.

22. Judd RM, Lugo-Olivieri $\mathrm{CH}$, Arai $\mathrm{M}$, et al. Physiological basis of myocardial contrast enhancement in fast magnetic resonance images of 2-day-old reperfused canine infarcts. Circulation. Oct 1 1995;92(7):1902-1910.

23. Nijveldt R, Beek AM, Hofman MB, et al. Late gadolinium-enhanced cardiovascular magnetic resonance evaluation of infarct size and microvascular obstruction in optimally treated patients after acute myocardial infarction. J Cardiovasc Magn Reson. 2007;9(5):765-770.

24. Santoro GM, Valenti R, Buonamici P, et al. Relation between ST-segment changes and myocardial perfusion evaluated by myocardial contrast echocardiography in patients with acute myocardial infarction treated with direct angioplasty. Am J Cardiol. Oct 15 1998;82(8):932-937.

25. Choi CJ, Haji-Momenian S, Dimaria JM, et al. Infarct involution and improved function during healing of acute myocardial infarction: the role of microvascular obstruction. J Cardiovasc Magn Reson. 2004;6(4):917-925.

26. Gerber $\mathrm{BL}$, Rochitte $\mathrm{CE}$, Melin JA, et al. Microvascular obstruction and left ventricular remodeling early after acute myocardial infarction. Circulation. Jun 13 2000;101(23):2734-2741.

27. Nijveldt R, Beek AM, Hirsch A, et al. Functional recovery after acute myocardial infarction: comparison between angiography, electrocardiography, and cardiovascular magnetic resonance measures of microvascular injury. J Am Coll Cardiol. Jul 15 2008;52(3):181-189.

28. Wu KC, Zerhouni EA, Judd RM, et al. Prognostic significance of microvascular obstruction by magnetic resonance imaging in patients with acute myocardial infarction. Circulation. Mar 3 1998;97(8):765-772.

29. Lund GK, Stork A, Saeed M, et al. Acute myocardial infarction: evaluation with first-pass enhancement and delayed enhancement MR imaging compared with 201TI SPECT imaging. Radiology. Jul 2004;232(1):49-57.

30. Nijveldt R, Hofman MB, Hirsch A, et al. Assessment of microvascular obstruction and prediction of short-term remodeling after acute myocardial infarction: cardiac MR imaging study. Radiology. Feb 2009;250(2):363-370.

31. Rochitte CE, Lima JA, Bluemke DA, et al. Magnitude and time course of microvascular obstruction and tissue injury after acute myocardial infarction. Circulation. Sep 8 1998;98(10):1006-1014.

32. Villanueva FS. Myocardial contrast echocardiography in acute myocardial infarction. Am J Cardiol. Nov 18 2002;90(10A):38J-47J.

33. Wu KC, Kim RJ, Bluemke DA, et al. Quantification and time course of microvascular obstruction by contrast-enhanced echocardiography and magnetic resonance imaging following acute myocardial infarction and reperfusion. J Am Coll Cardiol. Nov 15 1998;32(6):1756-1764.

34. Galiuto L, Gabrielli FA, Lombardo A, et al. Reversible microvascular dysfunction coupled with persistent myocardial dysfunction: implications for post-infarct left ventricular remodelling. Heart. May 2007;93(5):565-571.

35. Fishbein MC, J YR, Lando $U$, et al. The relationship of vascular injury and myocardial hemorrhage to necrosis after reperfusion. Circulation. Dec 1980;62(6):1274-1279.

36. Garcia-Dorado D, Theroux P, Solares J, et al. Determinants of hemorrhagic infarcts. Histologic observations from experiments involving coronary occlusion, coronary reperfusion, and reocclusion. Am J Pathol. Aug 1990;137(2):301-311.

37. Kloner RA, Rude RE, Carlson N, et al. Ultrastructural evidence of microvascular damage and myocardial cell injury after coronary artery occlusion: which comes first? Circulation. Nov 1980;62(5):945952. 
38. Asanuma T, Tanabe K, Ochiai K, et al. Relationship between progressive microvascular damage and intramyocardial hemorrhage in patients with reperfused anterior myocardial infarction: myocardial contrast echocardiographic study. Circulation. Jul 15 1997;96(2):448-453.

39. Pislaru SV, Barrios L, Stassen T, et al. Infarct size, myocardial hemorrhage, and recovery of function after mechanical versus pharmacological reperfusion: effects of lytic state and occlusion time. Circulation. Jul 15 1997;96(2):659-666.

40. Lotan CS, Miller SK, Cranney GB, et al. The effect of postinfarction intramyocardial hemorrhage on transverse relaxation time. Magn Reson Med. Feb 1992;23(2):346-355.

41. Basso C, Corbetti F, Silva C, et al. Morphologic validation of reperfused hemorrhagic myocardial infarction by cardiovascular magnetic resonance. Am J Cardiol. Oct 15 2007;100(8):1322-1327.

42. Beek AM, Nijveldt R, van Rossum AC. Intramyocardial hemorrhage and microvascular obstruction after primary percutaneous coronary intervention. Int J Cardiovasc Imaging. Jan 2010;26(1):49-55.

43. Ganame J, Messalli G, Dymarkowski S, et al. Impact of myocardial haemorrhage on left ventricular function and remodelling in patients with reperfused acute myocardial infarction. Eur Heart J. Jun 2009;30(12):1440-1449.

44. Mather AN, Fairbairn TA, Ball SG, et al. Reperfusion haemorrhage as determined by cardiovascular $\mathrm{MRI}$ is a predictor of adverse left ventricular remodelling and markers of late arrhythmic risk. Heart doi:10.1136/hrt.2010.202028 Nov 42010.

45. Schroder R. Prognostic impact of early ST-segment resolution in acute ST-elevation myocardial infarction. Circulation. Nov 23 2004;110(21):e506-510.

46. Feldman LJ, Coste $\mathrm{P}$, Furber A, et al. Incomplete resolution of ST-segment elevation is a marker of transient microcirculatory dysfunction after stenting for acute myocardial infarction. Circulation. Jun 3 2003;107(21):2684-2689.

47. Buller CE, Fu Y, Mahaffey KW, et al. ST-segment recovery and outcome after primary percutaneous coronary intervention for ST-elevation myocardial infarction: insights from the Assessment of Pexelizumab in Acute Myocardial Infarction (APEX-AMI) trial. Circulation. Sep 23 2008;118(13):13351346.

48. Dong J, Ndrepepa G, Schmitt C, et al. Early resolution of ST-segment elevation correlates with myocardial salvage assessed by Tc-99m sestamibi scintigraphy in patients with acute myocardial infarction after mechanical or thrombolytic reperfusion therapy. Circulation. Jun 25 2002;105(25):29462949.

49. Sciagra R, Parodi G, Migliorini A, et al. ST-segment analysis to predict infarct size and functional outcome in acute myocardial infarction treated with primary coronary intervention and adjunctive abciximab therapy. Am J Cardiol. Jan 1 2006;97(1):48-54.

50. van 't Hof AW, Liem A, de Boer MJ, et al. Clinical value of 12-lead electrocardiogram after successful reperfusion therapy for acute myocardial infarction. Zwolle Myocardial infarction Study Group. Lancet. Aug 30 1997;350(9078):615-619.

51. Lund GK, Stork A, Muellerleile K, et al. Prediction of left ventricular remodeling and analysis of infarct resorption in patients with reperfused myocardial infarcts by using contrast-enhanced MR imaging. Radiology. Oct 2007;245(1):95-102.

52. Orn S, Manhenke C, Greve OJ, et al. Microvascular obstruction is a major determinant of infarct healing and subsequent left ventricular remodelling following primary percutaneous coronary intervention. Eur Heart J. Jun 62009.

53. Larose $\mathrm{E}$, Rodes-Cabau J, Pibarot $\mathrm{P}$, et al. Predicting late myocardial recovery and outcomes in the early hours of ST-segment elevation myocardial infarction traditional measures compared with microvascular obstruction, salvaged myocardium, and necrosis characteristics by cardiovascular magnetic resonance. J Am Coll Cardiol. Jun 1 2010;55(22):2459-2469.

54. Shapiro MD, Nieman K, Nasir K, et al. Utility of cardiovascular magnetic resonance to predict left ventricular recovery after primary percutaneous coronary intervention for patients presenting with acute ST-segment elevation myocardial infarction. Am J Cardiol. Jul 15 2007;100(2):211-216. 
55. Tarantini G, Razzolini R, Cacciavillani L, et al. Influence of transmurality, infarct size, and severe microvascular obstruction on left ventricular remodeling and function after primary coronary angioplasty. Am J Cardiol. Oct 15 2006;98(8):1033-1040.

56. Nijveldt R, van der Vleuten PA, Hirsch A, et al. Early electrocardiographic findings and MR imagingverified microvascular injury and myocardial infarct size. JACC Cardiovasc Imaging. Oct 2009;2(10):1187-1194.

57. Haeck JD, Koch KT, Bilodeau L, et al. Randomized comparison of primary percutaneous coronary intervention with combined proximal embolic protection and thrombus aspiration versus primary percutaneous coronary intervention alone in ST-segment elevation myocardial infarction: the PREPARE (PRoximal Embolic Protection in Acute myocardial infarction and Resolution of ST-Elevation) study. JACC Cardiovasc Interv. Oct 2009;2(10):934-943.

58. Haeck JD, Kuijt WJ, Koch KT, et al. Infarct size and left ventricular function in the PRoximal Embolic Protection in Acute myocardial infarction and Resolution of ST-segment Elevation (PREPARE) trial: ancillary cardiovascular magnetic resonance study. Heart. Feb 2009;96(3):190-195.

59. Haeck JD, Verouden NJ, Kuijt WJ, et al. Impact of early, late, and no ST-segment resolution measured by continuous ST Holter monitoring on left ventricular ejection fraction and infarct size as determined by cardiovascular magnetic resonance imaging. J Electrocardiol. Jan-Feb 2011;44(1):36-41.

60. Basso C, Rizzo S, Thiene G. The metamorphosis of myocardial infarction following coronary recanalization. Cardiovasc Pathol. Jan-Feb 2010;19(1):22-28.

61. Fieno DS, Kim RJ, Chen EL, et al. Contrast-enhanced magnetic resonance imaging of myocardium at risk: distinction between reversible and irreversible injury throughout infarct healing. J Am Coll Cardiol. Nov 15 2000;36(6):1985-1991.

62. Kim RJ, Fieno DS, Parrish TB, et al. Relationship of MRI delayed contrast enhancement to irreversible injury, infarct age, and contractile function. Circulation. Nov 9 1999;100(19):1992-2002.

63. Aldrich HR, Wagner NB, Boswick J, et al. Use of initial ST-segment deviation for prediction of final electrocardiographic size of acute myocardial infarcts. Am J Cardiol. Apr 1 1988;61(10):749-753.

64. Graham MM, Faris PD, Ghali WA, et al. Validation of three myocardial jeopardy scores in a population-based cardiac catheterization cohort. Am Heart J. Aug 2001;142(2):254-261.

65. Ortiz-Perez JT, Meyers SN, Lee DC, et al. Angiographic estimates of myocardium at risk during acute myocardial infarction: validation study using cardiac magnetic resonance imaging. Eur Heart J. Jul 2007;28(14):1750-1758.

66. Aletras AH, Tilak GS, Natanzon A, et al. Retrospective determination of the area at risk for reperfused acute myocardial infarction with T2-weighted cardiac magnetic resonance imaging: histopathological and displacement encoding with stimulated echoes (DENSE) functional validations. Circulation. Apr 18 2006;113(15):1865-1870.

67. Berry C, Kellman P, Mancini C, et al. Magnetic resonance imaging delineates the ischemic area at risk and myocardial salvage in patients with acute myocardial infarction. Circ Cardiovasc Imaging. Sep 1 2010;3(5):527-535.

68. Friedrich MG, Abdel-Aty $\mathrm{H}$, Taylor A, et al. The salvaged area at risk in reperfused acute myocardial infarction as visualized by cardiovascular magnetic resonance. J Am Coll Cardiol. Apr 22 2008;51(16):1581-1587.

69. Masci PG, Ganame J, Strata E, et al. Myocardial salvage by CMR correlates with LV remodeling and early ST-segment resolution in acute myocardial infarction. JACC Cardiovasc Imaging. Jan 2010;3(1):45-51.

70. Ubachs JF, Engblom H, Erlinge D, et al. Cardiovascular magnetic resonance of the myocardium at risk in acute reperfused myocardial infarction: comparison of T2-weighted imaging versus the circumferential endocardial extent of late gadolinium enhancement with transmural projection. J Cardiovasc Magn Reson. 2010;12(29):18.

71. Wright J, Adriaenssens T, Dymarkowski S, et al. Quantification of myocardial area at risk with T2weighted CMR: comparison with contrast-enhanced CMR and coronary angiography. JACC Cardiovasc Imaging. Jul 2009;2(7):825-831. 
72. Stone GW, Witzenbichler B, Guagliumi G, et al. Bivalirudin during primary PCl in acute myocardial infarction. N Engl J Med. May 22 2008;358(21):2218-2230.

73. Kim HW, Farzaneh-Far A, Kim RJ. Cardiovascular magnetic resonance in patients with myocardial infarction: current and emerging applications. J Am Coll Cardiol. Dec 29 2009;55(1):1-16.

74. Burns RJ, Gibbons RJ, Yi Q, et al. The relationships of left ventricular ejection fraction, end-systolic volume index and infarct size to six-month mortality after hospital discharge following myocardial infarction treated by thrombolysis. J Am Coll Cardiol. Jan 2 2002;39(1):30-36.

75. Wu E, Ortiz JT, Tejedor $\mathrm{P}$, et al. Infarct size by contrast enhanced cardiac magnetic resonance is a stronger predictor of outcomes than left ventricular ejection fraction or end-systolic volume index: prospective cohort study. Heart. Jun 2008;94(6):730-736.

76. Lim SY, Hausenloy DJ, Arjun S, et al. Mitochondrial cyclophilin-D as a potential therapeutic target for post-myocardial infarction heart failure. J Cell Mol Med. Dec 92010.

77. Staat P, Rioufol G, Piot C, et al. Postconditioning the human heart. Circulation. Oct 4 2005;112(14):2143-2148.

78. Vanagt WY, Cornelussen RN, Baynham TC, et al. Pacing-induced dyssynchrony during early reperfusion reduces infarct size. J Am Coll Cardiol. May 1 2007;49(17):1813-1819.

79. Bai $X, Y a n Y$, Song $Y H$, et al. Both cultured and freshly isolated adipose tissue-derived stem cells enhance cardiac function after acute myocardial infarction. Eur Heart J. Feb 2010;31(4):489-501.

80. Engelen DJ, Gressin V, Krucoff MW, et al. Usefulness of frequent arrhythmias after epicardial recanalization in anterior wall acute myocardial infarction as a marker of cellular injury leading to poor recovery of left ventricular function. Am J Cardiol. Nov 15 2003;92(10):1143-1149.

81. Majidi M, Kosinski AS, Al-Khatib SM, et al. Reperfusion ventricular arrhythmia 'bursts' predict larger infarct size despite TIMI 3 flow restoration with primary angioplasty for anterior ST-elevation myocardial infarction. Eur Heart J. Apr 2009;30(7):757-764.

82. Roes SD, Borleffs CJ, van der Geest RJ, et al. Infarct tissue heterogeneity assessed with contrastenhanced MRI predicts spontaneous ventricular arrhythmia in patients with ischemic cardiomyopathy and implantable cardioverter-defibrillator. Circ Cardiovasc Imaging. May 2009;2(3):183-190.

83. Kim HW, Klem I, Shah DJ, et al. Unrecognized non-Q-wave myocardial infarction: prevalence and prognostic significance in patients with suspected coronary disease. PLoS Med. Apr 21 2009;6(4):e1000057. 
Summary 
Mortality from acute myocardial infarction (AMI) has declined the last decades, but remains high. Timely epicardial reperfusion significantly reduces infarct size but reperfusion is not always established at the tissue level. The mechanisms of this microvascular obstruction (MVO) remain incompletely understood. Reperfusion may paradoxically lead to additional myocyte death (reperfusion inury) and intramyocardial hemorrhage (IMH), because of leakage of erythrocytes from severely damaged microvasculature. Myocyte death, MVO and IMH are strongly related, time-sensitive phenomena, but MVO and IMH lag behind myocyte death. Infarct size is a well known predictor of outome after AMI but conflicting results have been published about the clinical significance of MVO and IMH beyond infarct size. With different techniques, cardiovascular magnetic resonance (CMR) provides a comprehensive, multifaceted investigation of the heart in AMI. The optimal timing of imaging of MVO after contrast administration and the dynamic changes in its appearance in relation to the underlying infarct characteristics and remodeling are not well known. ST segment resolution (STR) on the electrocardiogram after reperfusion is an accepted marker for myocardial reperfusion and useful for early risk stratification. However, the exact relation of STR with underlying infarct characteristics and remodeling as assessed with CMR is not well known. For the evaluation of additional infarct size limiting therapies, there is a need to accurately measure the area at risk (AAR) and myocardial salvage. Despite an increased interest in angiographic, electrocardiographic and CMR techniques for measuring the AAR, validation in humans is still limited. In this thesis, characteristics of injury in reperfused AMI were assessed with CMR and their clinical significance in relation to remodeling was investigated. In addition, the relation of STR with these infarct characteristics and ventricular remodeling was investigated. Concerning AAR measurement, the performance of different electrocardiographic, angiographic, and CMR methods was evaluated using established physiological concepts.

In chapter 2, the underlying pathophysiology of myocardial ischemia with an emphasis on MVO and current techniques to detect MVO are reviewed.

Chapter 3 describes an optimized single breathhold inversion recovery gradient echo pulse sequence for measuring MVO 2 minutes after contrast administration (early MVO). In this study, traditional delayed enhancement (DE) CMR 10 minutes after contrast administration was used to detect late MVO. Cine CMR was used to assess left ventricular (LV) volumes and ejection fraction (EF) at 5 days and 3 months after admission. The prevalence of early MVO was higher than late MVO (63\% vs. 54\%, $p=0.008$ ). The extent of MVO decreased from early to late imaging $(4.3 \pm 3.2 \%$ vs. $1.8 \% \pm 1.8 \%, p<0.001)$ and this decrement showed a heterogeneous pattern. At baseline, patients without MVO (early and late) had a higher LVEF than patients with persistent late MVO (56 $\pm 7 \%$ vs. $48 \pm 7 \%, p<0.001)$ and LVEF was intermediate in patients with early MVO but late MVO disappearance ( $54 \pm 6 \%$ ). During follow-up, LVEF improved in all three subgroups but remained intermediate in 
patients with late MVO disappearance. Our approach of imaging MVO early and late after contrast administration allowed the discrimination of a subgroup of patients with early but late disappearance of MVO. These patients had infarct sizes and remodeling parameters that were intermediate between patients without any MVO and patients who had both early and late MVO.

The prevalence and clinical implications of $\mathrm{MVO}$ and $\mathrm{IMH}$ in a group of reperfused first AMI patients were investigated in chapter 4. T2-weighted (T2W), cine and DE-CMR were performed at 5 days and 3 months after admission to assess IMH, LV function and late MVO. MVO was observed in 54\% and IMH in $43 \%$ of patients. All patients with IMH had MVO, but not vice versa. MVO and IMH were always located subendocardially and correlated strongly $(r=0.8, p<0.001)$. Pre-PCI TIMI 3 flow was only observed in patients without MVO and IMH. Infarct size and impairment of systolic function were largest in patients with both $\mathrm{MVO}$ and IMH, smallest in patients without MVO and IMH and intermediate in patients with MVO but without IMH, both at baseline and follow-up. Infarct size was the only independent predictor of LV remodeling.

The relation of early post-percutaneous coronary intervention (PCI) STR with underlying infarct characteristics and adverse remodeling was investigated in Chapter 5. For this study, 8 different STR methods (i.e. relative STR (\%) and residual absolute ST segment deviation (STD, $\mathrm{mm}$ ), summed and single lead), were evaluated at 30 and 60 minutes after $\mathrm{PCl}$. Cine and $\mathrm{DE}-\mathrm{CMR}$ were performed at 5 days and 3 months after admission. Using $K$-means cluster analysis, three CMR risk groups (low, intermediate, high) for adverse remodeling were identified based on combinations of infarct size, infarct transmurality and late MVO. Infarct size was the main determinant of adverse remodeling (low LVEF) after 3 months. Of all tested STR variables worst lead residual STD 30 minutes after reperfusion allowed accurate identification of patients at high risk for adverse remodeling after 3 months, which was mainly related to IS rather than transmurality or MVO.

In chapter 6, the performance of electrocardiographic (Aldrich), angiographic (BARI and APPROACH), and CMR methods (T2W hyperintensity and infarct endocardial surface area (ESA) by DE-CMR) for measuring the AAR were evaluated, using the following established physiological concepts as a reference standard: 1) AAR $\geq$ infarct size, 2) in transmural infarcts, AAR should approach infarct size, 3) the correlation between AAR and infarct size should increase as infarct transmurality increases, 4) myocardial salvage [(AAR - infarct size)/AAR x 100] should be inversely related to infarct transmurality, following the inverse line of identity. For measuring $A A R, C M R$ methods were better than angiographic methods, which were better than electrocardiographic. Overall, ESA by DE-CMR appeared to be the best method for measuring AAR in vivo.

Chapter 7 discusses the main findings of this thesis in the light of existing evidence or controversy. Future perspectives are given in the end. 


\section{Conclusions}

CMR allows accurate assessment of injury of reperfused AMI, showing that MVO and $\mathrm{IMH}$ are prevalent and strongly related phenomena. Furthermore, our results showed that infarct size was the most important predictor of adverse remodeling after 3 months. Worst lead residual STD 30 minutes after reperfusion allows accurate identification of patients at high risk for adverse remodeling, which was mainly related to IS rather than transmurality or MVO. For measuring the AAR, CMR methods performed better than angiographic methods, which were better than electrocardiographic methods. 
Samenvatting 
Hoewel de sterfte t.g.v. het acute myocard infarct (AMI) de laatste decennia is gedaald, blijft deze hoog. Vroegtijdige epicardiale reperfusie beperkt de infarctgrootte, maar op weefselniveau wordt optimale reperfusie niet altijd bereikt. Het mechanisme van deze microvasculaire obstructie (MVO) is onvolledig bekend. Reperfusie kan paradoxaal leiden tot additionele spiercelsterfte (reperfusieschade) en intramyocardiale bloeding $(\mathrm{IMH})$, doordat erytrocyten uit de ernstig beschadigde bloedvaatjes 'lekken'. Spiercelsterfte, MVO en IMH zijn sterk aan elkaar gerelateerde en tijdsafhankelijke fenomenen, maar bekend is dat MVO en IMH pas ontstaan na het optreden van spiercelsterfte. De prognose na een AMI wordt in belangrijke mate bepaald door de infarctgrootte. Studies die de onafhankelijke invloed van MVO en IMH op prognose onderzoeken zijn echter niet éénduidig. Cardiovasculaire magnetische resonantie beeldvorming (CMR) maakt uitgebreid en veelzijdig onderzoek van het geïnfarceerde hart mogelijk. Na toediening van een contrastmiddel zijn het optimale tijdstip voor het opsporen en de dynamische veranderingen van MVO niet goed bekend. Hoewel ST segment resolutie (STR) op het elektrocardiogram na reperfusie een geaccepteerde maat is voor de kwaliteit van reperfusie en gebruikt wordt voor vroege risicostratificatie, is de relatie van STR met onderliggende infarctkarakteristieken en cardiale functieveranderingen (adverse remodeling) na een AMI onvolledig bekend. Voor de ontwikkeling en evaluatie van nieuwe therapieën die infarctgrootte verder kunnen beperken, is nauwkeurige meting van de area at risk (AAR) en myocardial salvage nodig. Hoewel er een toenemende belangstelling is in electrocardiografische, angiografische en CMR methoden om de AAR te meten, is klinische validatie nog beperkt. In dit proefschrift worden verschillende infarctkarakteristieken bepaald m.b.v. CMR en hun relatie met STR en adverse remodeling onderzocht. Tevens wordt onderzocht hoe bruikbaar verschillende electrocardiografische, angiografische en CMR methoden zijn om de AAR te meten, door ze onderling te vergelijken aan de hand van enkele fysiologische principes van infarctuitbreiding.

Hoofdstuk 2 geeft een overzicht van de onderliggende pathofysiologie van myocardischemie met de nadruk op MVO en de huidige methoden om MVO te diagnosticeren.

Hoofdstuk 3 beschrijft een geoptimaliseerde, in één adempauze verkregen, inversion recovery gradient echo pulse sequence voor de detectie van MVO 2 minuten na contrasttoediening (vroege MVO). Na 10 minuten vond tevens traditionele delayed enhancement (DE) CMR plaats voor de detectie van late MVO. D.m.v. cine CMR werden linker ventrikel (LV) volumina en ejectie fractie (EF) 5 dagen en 3 maanden na ziekenhuisopname gemeten. De prevalentie van vroege MVO was hoger dan van late MVO (63\% vs. 54\%, $p=0.008$ ). De grootte van MVO nam af van vroeg naar laat $(4.3 \pm 3.2 \%$ vs. $1.8 \% \pm 1.8 \%, p<0.001)$ en vertoonde een heterogeen patroon. De uitgangs-EF was hoger bij patiënten zonder MVO (vroeg en laat) dan bij patiënten met persisterende late MVO (56 $\pm 7 \%$ vs. $48 \pm 7 \%, p<0.001$ ) en intermediair bij patiënten met wel vroege maar geen late MVO (54 $\pm 6 \%$ ). De follow-up EF 
verbeterde in alle 3 groepen maar bleef intermediair in de patiënten bij wie late MVO verdween. Door deze strategie konden patiënten worden onderscheiden die wel vroege maar geen late MVO hadden (verdwijning) en bij wie infarctgrootte en adverse remodeling intermediair waren tussen patiënten zonder MVO en patiënten met zowel vroege als late MVO.

De prevalentie en klinische rol van MVO en IMH in een groep patiënten met een gereperfundeerd eerste AMI werden onderzocht in hoofdstuk 4. MVO, IMH en LV functie werden 5 dagen en 3 maanden na ziekenhuisopname bepaald d.m.v. DE-, T2-gewogen (T2W) en cine CMR. De prevalentie van MVO en IMH was 54\% en $43 \%$. Alle patiënten met IMH hadden MVO maar niet vice versa. MVO en IMH waren sterk gecorreleerd $(r=0.8, p<0.001)$ en altijd subendocardiaal gelokaliseerd. TIMI 3 flow vóór percutaneous coronary intervention $(\mathrm{PCl})$ werd alleen waargenomen bij patiënten zonder MVO en IMH. De begin- en follow-up infarctgrootte en LV disfunctie waren het grootst in patiënten met zowel MVO als IMH, het kleinst in patiënten zonder MVO en IMH en intermediair in patiënten met MVO maar zonder IMH. Infarctgrootte was de enige onafhankelijke voorspeller van adverse remodeling.

De relatie van vroege STR na $\mathrm{PCl}$ met onderliggende infarctkarakteristieken en adverse remodeling werd onderzocht in hoofdstuk $\mathbf{5}$. In deze studie werden 8 verschillende methoden om STR te meten (proportionele STR (\%) en absolute ST segment deviatie (STD, mm), cumulatief en in één afleiding), geëvalueerd 30 en 60 minuten na $\mathrm{PCl}$. Cine en $\mathrm{DE}-\mathrm{CMR}$ werden verricht, 5 dagen en 3 maanden na ziekenhuisopname. Gebaseerd op infarctgrootte, MVO en infarcttransmuraliteit werden m.b.v. k-means clusteranalyse, 3 CMR groepen onderscheiden met een verschillend risico op adverse remodeling (laag, intermediair, en hoog). Infarctgrootte was de belangrijkste determinant van adverse remodeling (low LVEF) na 3 maanden. Absolute STD in de 'slechtste' afleiding, 30 minuten na PCl, identificeerde het beste patiënten met een verhoogd risico op adverse remodeling en was sterker gerelateerd aan infarctgrootte dan MVO en transmuraliteit.

In hoofdstuk 6 werden electrocardiografische (Aldrich), angiografische (BARI, APPROACH) en CMR methoden (T2W hyperintensiteit, endocardiaal infarct oppervlak (ESA)) om de AAR te meten, onderling relatief vergeleken aan de hand van enkele fysiologische basisprincipes van infarctuitbreiding als referentie standaard: 1) $A A R \geq$ infarctgrootte, 2) in transmurale infarcten is de AAR ongeveer gelijk aan infarctgrootte, 3) de correlatie tussen AAR en infarctgrootte neemt toe als infarcttransmuraliteit toeneemt, 4) myocardial salvage [(AAR - infarctgrootte)/AAR x 100] is omgekeerd evenredig aan infarcttransmuraliteit en ligt dichtbij de identiteitslijn. CMR methoden waren beter dan angiografische, die weer beter waren dan electrocardiografische methoden om de AAR te meten. Over het algemeen, bleek ESA d.m.v. DE-CMR de beste methode om AAR in vivo te bepalen.

Hoofdstuk 7 beschrijft de belangrijkste bevindingen uit dit proefschrift en beschouwt deze tegen het licht van de huidige wetenschappelijke literatuur. 


\section{Conclusies}

$\mathrm{CMR}$ is in staat nauwkeurig de schade en karakteristieken van een gereperfundeerd acuut myocardinfarct te bepalen en toont aan dat MVO en IMH vaak voorkomen en sterk zijn gecorreleerd. Bovendien blijkt uit onze studies dat infarctgrootte de belangrijkste voorspeller is van adverse remodeling na 3 maanden. Absolute STD in de slechtste afleiding 30 minuten na $\mathrm{PCl}$ identificeerde het beste patiënten met een verhoogd risico op adverse remodeling na 3 maanden en was sterker gerelateerd aan infarctgrootte dan MVO en infarcttransmuraliteit. Om de area at risk te meten, zijn CMR methoden beter dan angiografische, die weer beter zijn dan electrocardiografische methoden. 
Dankwoord 
In 2006 begon ik dit proefschrift aan een nieuw, zelf ontworpen bureau dat door mijn mijn handige schoonbroer Cees werd gemaakt. De strakke lijnen, de diepe warmrode kleur van Jatobahout, de subtiele metalen verbindingsstukken geven het een moderne en klassieke uitstraling. Hoewel in de loop der jaren verdwenen onder stapels artikelen en aantekeningen, is het de spil gebleven in de organisatie van mijn proefschrift. Natuurlijk was dit proefschrift niet mogelijk geweest zonder de vele personen die mij de afgelopen jaren hebben geholpen. Ik bedank mijn beide promotoren, prof. dr. Anton P.M. Gorgels en prof. dr. Johannes L. Waltenberger.

Beste Ton, jouw idee voor de MAST studie vormde de basis van dit proefschrift. Als collega was ik een ongewone promovendus. Mede door jouw vertrouwen, heb ik veel zelfstandig kunnen doen. Ik ken je als een toegankelijke, warme persoonlijkheid en bewonder je kennis van de elektrocardiografie. Ik ben je opvattingen over geneeskunst steeds meer gaan waarderen: de weg naar genezing begint bij een goede anamnese en zorgvuldig lichamelijk onderzoek.

Beste Johannes, ik bewonder je als 'international professional', iemand die zijn carrière buiten de eigen landsgrenzen succesvol opbouwt. Dank voor de uitnodiging te spreken over microvasculaire obstructie op het ESC congres in München in 2008. Het heeft geleid tot het overzichtsartikel dat nu als één van de hoofdstukken van dit proefschrift dient.

De leden van de beoordelingscommissie, Prof. dr. Joachim E. Wildberger (voorzitter), Prof. dr. Harry J.G.M. Crijns, Prof. dr. Mat J. Daemen, Prof. dr. Frank E. Rademakers en Prof. dr. Albert C. van Rossum ben ik erkentelijk voor het beoordelen van dit manuscript. Voor het opponeren bedank ik tevens Prof. dr. Frits W. Prinzen en Prof. dr. Hein J.J. Wellens, die aan de basis heeft gestaan van mijn opleiding tot cardioloog en eerste stappen in wetenschappelijk onderzoek.

Miguel Lemmert, hartelijk dank voor je hulp in de beginfase van de MAST studie en de eerste analyses. Nog even doorzetten en je eigen promotie is weldra daar.

Ook bedank ik Petra Erkens, die vele patiënten includeerde, case record forms bijhield en het SPSS moederbestand creëerde. Succes met je eigen promotie.

Begonnen als WESP student, heeft ook Martijn Smulders een belangrijke bijdrage geleverd. Dank voor de vele analyses. Ik zie er naar uit jouw promotietraject de komende jaren te begeleiden. Mathijs Versteylen, ons artikel kende een lange reis met de eindbestemming bijna in het zicht. Dank voor jouw volhardendheid.

Beste Valéria, ik ben je zeer dankbaar voor de statistische ondersteuning en zorgvuldige argumenten. Je Braziliaanse temperament is hartverwarmend.

Ik bedank collega Simon Schalla voor het kritisch beoordelen van de artikelen. Ik hoop dat we CMR in het MUMC tot nog grotere hoogte kunnen brengen.

I thank Prof. dr. R.J. Kim, professor of Medicine at Duke University Medical Center. Dear Ray, I am very grateful for the many hours we have spent during phone conferences and visits. Your knowledge and thoughts on research and scientific writing have been very inspiring. I look forward to our continuing cooperation. 
Beste Harry, dank voor je ondersteuning en dat je als afdelingshoofd mede mogelijk hebt gemaakt dat ik mijn tijd kon pakken om dit proefschrift af te ronden.

Beste Miel, door jou werd ik geënthousiasmeerd in cardiovasculaire beeldvorming. Je blijft voor mij de beste echocardiografist van Nederland. Als opleider en overdrager van kennis zijn de woorden 'trans' en 'ducere' je op het lijf geschreven.

Beste Dirk, ik bewonder je doorzettingsvermogen. Jij wordt de eerste Nederlandse hoogleraar ICCU geneeskunde. Dank voor alle bijzondere momenten op de werkvloer en op de fiets.

De verpleging van de ICCU en verpleegafdeling cardiologie, in het bijzonder Jean Partouns, dank ik voor de prettige samenwerking de afgelopen jaren en zorg voor de patiënten van de MAST studie. Jean, geniet van je welverdiende pensioen.

Dank aan de laboranten van de afdeling radiologie, speciaal Liesbeth en Kim, voor de CMR onderzoeken. Met plezier heb ik de afgelopen jaren samengewerkt met radiologen Riël Snoep, Tim Leiner en Suzanne Gommers.

Dank aan alle laboranten echocardiografie, in het bijzonder moederlaborant Andrea Palmans-Meulemans en 'Sjwens' Jos Habets, voor jullie bijdrage. Door de goede sfeer die jullie altijd weer weten te creëren op de echokamer ben ik er graag.

Ik bedank de assistenten cardiologie voor hun bijdrage de afgelopen jaren en mijn collega cardiologen, die af en toe een tandje bij moesten zetten in de kliniek, zodat ik dit proefschrift kon afronden.

Nicoline Nanninga en Nadine Hofman-Servais dank ik voor de secretariële ondersteuning en Anita Kaemingk voor de in- en uitwendige opmaak van dit boek.

Mijn goede vrienden, speciaal Marcel en Tom, bedank ik voor hun geduld de afgelopen jaren. Ik zie uit om weer samen met onze gezinnen erop uit te trekken.

Beste Jan en Henny, dank voor jullie luisterende oor en interesse in de bezigheden van jullie schoonzoon de afgelopen jaren.

Lieve Paul en Saskia, een broer als bankier en schoonzus werkzaam bij een fameuze uitgever geeft gelukkig weer andere praat tijdens familiebijeenkomsten.

Lieve pa en ma, de waarden en normen die jullie meegaven vormen de basis voor ons succes. Dank voor jullie onvoorwaardelijke liefde en steun. Het warme nest van vroeger en nu, zal altijd een voorbeeld voor ons blijven.

Lieve Netty, liefde van mijn leven. Zonder jou was dit alles niet mogelijk geweest. Promoveren naast een baan als cardioloog is geen sinecure gebleken. De afgelopen jaren zorgde jij dat ons gezinnetje draaide. Wat ben je toch een ongelooflijk stabiele en sterke persoonlijkheid en wat prijs ik mij gelukkig het leven met jou te delen. En wat zijn we gezegend met onze twee kinderen, die het in alle opzichten fantastisch doen. Lieve Sam en Merel, papa is ongelooflijk trots op alles wat jullie op eigen kracht doen, op school, met jullie gitaarspel en op het hockeyveld, maar wat gaat de tijd toch snel. Gisteren nog in mijn armen, nu al of bijna naar de middelbare school. Vanaf morgen ga ik het bewuster meemaken. Jullie zijn mijn drijvende kracht. 


\section{Curriculum vitae}


CURRICULUM VITAE 
Date of birth

Place of birth

1979-1985

1985-1986

1986-1994

1995

1997-2003

1998-1996

2001

2003

2003-present

2004

2006

2008
8 August 1967

's-Hertogenbosch, the Netherlands

Gymnasium $\beta$, Stedelijk Gymnasium 's-Hertogenbosch, the Netherlands

Pharmacy, University of Groningen, the Netherlands

Medicine, Maastricht University, the Netherlands

Military service, first lieutanent and general physcian

Cardiology training, Maastricht University Medical Center, the Netherlands (Prof. dr. H.J.J. Wellens and Prof. dr.

H.J.G.M. Crijns)

Internal Medicine training Maasland hospital (currently

Orbis Medisch Centrum), Sittard, the Netherlands

Research fellow at Indiana Center for Vascular Biology

and Medicine, Krannert Institute of Cardiology, Indiana

University, Indianapolis, USA (Prof. dr. K.L. March)

Registered Cardiologist

Cardiologist at Maastricht University Medical Center (noninvasive cardiovascular imaging, supervisor of coronary care unit and cardiology emergency department)

Training in Cardiovascular Magnetic Resonance Imaging at Royal Brompton Hospital, London, UK

(Prof. dr. D. Pennell). Euro-CMR exam in 2005

Start of the MAST-study at the department of Cardiology,

Maastricht University Medical Center, the Netherlands, on which this thesis is based (Prof. dr. A.P.M. Gorgels and Prof. dr. J.L. Waltenberger)

ESC accreditation in Intensive and Acute Cardiac Care 


\section{List of publications}


1. Giant atria in a patient with systemic lupus erythematosus. Weijs B, van Paassen $P$, Bekkers SC. Neth J Med. 2010 Nov; 68 (11): 378.

2. Images in cardiovascular medicine: Retrograde flow in the descending thoracic aorta: magnetic resonance imaging of an apicoaortic conduit. Jaarsma C, Nijs J, Gommers S, Bekkers SC, Schalla S. Circulation. 2010 Jul 13; 122 (2): e12.

3. Clinical implications of microvascular obstruction and intramyocardial haemorrhage in acute myocardial infarction using cardiovascular magnetic resonance imaging. Bekkers SC, Smulders MW, Passos VL, Leiner T, Waltenberger J, Gorgels AP, Schalla S. Eur Radiol. 2010 Nov; 20 (11): 2572-8.

4. Microvascular obstruction: underlying pathophysiology and clinical diagnosis. Bekkers SC, Yazdani SK, Virmani R, Waltenberger J. J Am Coll Cardiol. 2010 Apr 20; 55 (16): 164960.

5. Replacement and reactive myocardial fibrosis in idiopathic dilated cardiomyopathy: comparison of magnetic resonance imaging with right ventricular biopsy. Schalla $S$,

Bekkers SC, Dennert R, van Suylen RJ, Waltenberger J, Leiner T, Wildberger J, Crijns HJ, Heymans S. Eur J Heart Fail. 2010 Mar; 12 (3): 227-31.

6. RR-interval irregularity precedes ventricular fibrillation in ST elevation acute myocardial infarction. Lemmert ME, Majidi M, Krucoff MW, Bekkers SC, Crijns HJ, Wellens HJ, Kosinski AS, Gorgels AP. Heart Rhythm. 2010 Jan; 7 (1): 65-71.

7. Extensive injury after use of a mechanical cardiopulmonary resuscitation device. Wind $\mathrm{J}$, Bekkers SC, van Hooren L, van Heurn LW. Am J Emerg Med. 2009 Oct;27(8):1017.e1-2.

8. In vivo histology by cardiovascular magnetic resonance imaging. Delnoij T, van Suylen RJ, Cleutjens JP, Schalla S, Bekkers SC. Eur Heart J. 2009 Oct; 30 (20): 2492.

9. Detection and characteristics of microvascular obstruction in reperfused acute myocardial infarction using an optimized protocol for contrast-enhanced cardiovascular magnetic resonance imaging. Bekkers SC, Backes WH, Kim RJ, Snoep G, Gorgels AP, Passos VL, Waltenberger J, Crijns HJ, Schalla S. Eur Radiol. 2009; 19: 2904-2912.

10. Life after survival: long-term daily functioning and quality of life after an out-of-hospital cardiac arrest. Wachelder EM, Moulaert VR, van Heugten C, Verbunt JA, Bekkers SC, Wade DT. Resuscitation. 2009 May; 80 (5): 517-22.

11. Cardiac adaptation to pregnancy in women with a history of preeclampsia and a subnormal plasma volume. Andrietti S, Kruse AJ, Bekkers SC, Sep S, Spaanderman M, Peeters LL. Reprod Sci. 2008 Dec; 15 (10): 1059-65.

12. Images in cardiovascular medicine. Imaging of arrhythmogenic right ventricular cardiomyopathy.Winkens MH, Snoep G, Bekkers SC. Circulation. 2008 Sep 16;118(12):e158-9.

13. Reversible isolated left ventricular non-compaction? Eurlings LW, Pinto YM, Dennert RM, Bekkers SC. Int J Cardiol. 2009 Aug 14; 136 (2): e35-6.

14. Multivessel myocardial infarction. Bekkers SC, Lemmert ME, Schalla S, Snoep R, Crijns HJ, Gorgels AP. Neth Heart J. 2007 Dec; 15 (12): 424-5.

15. Cardiac cine MRI: comparison of $1.5 \mathrm{~T}$, non-enhanced 3.0 T and blood pool enhanced 3.0 T imaging. Gerretsen SC, Versluis B, Bekkers SC, Leiner T. Eur J Radiol. 2008 Jan; 65 (1): 80-5.

16. Hypothermia for out-of-hospital cardiac arrest survivors: a single-center experience. Bekkers SC, Eikemans BJ, Tieleman R, Braat SH, Dassen W, Partouns J, de Zwaan C, Crijns HJ, de Krom MC. Am J Emerg Med. 2007 Nov; 25 (9): 1078-80. 
17. Activity and Life After Survival of a Cardiac Arrest (ALASCA) and the effectiveness of an early intervention service: design of a randomised controlled trial. Moulaert VR, Verbunt JA, van Heugten CM, Bakx WG, Gorgels AP, Bekkers SC, de Krom MC, Wade DT. BMC Cardiovasc Disord. 2007 Aug 27; 7: 26.

18. Primary monophasic mediastinal, cardiac and pericardial synovial sarcoma: a young man in distress. de Zwaan C, Bekkers SC, van Garsse LA, Jansen RL, van Suylen RJ. Neth Heart J. 2007; 15 (6): 226-8.

19. A dancing thrombus in the right atrium going hand-in-hand with the electrocardiogram.van Opstal JM, Bekkers SC, Gorgels AP. Eur J Echocardiogr. 2008 Jan;9(1):80-1.

20. Late complication after closure of a ventricular septal rupture. Smedema JP, Bekkers SC, Snoep G. Cardiovasc J S Afr. 2006 Jul-Aug; 17 (4): 200-1.

21. Comprehensive assessment of left ventricular pseudo-aneurysms with magnetic resonance imaging.Smedema JP, Bekkers SC, Snoep G. Cardiovasc J S Afr. 2006 May-Jun; 17 (3): 130-4.

22. Ventricular pseudoaneurysm after subacute myocardial infarction. Bekkers SC, Borghans RA, Cheriex EC. Int J Cardiovasc Imaging. 2006 Dec; 22 (6): 791-5.

23. Images in cardiovascular medicine. Myocardial bridging. Bekkers SC, Leiner T. Circulation. 2006 Mar 7; 113 (9): e390-1.

24. Left ventricular pseudoaneurysm. Schalla S, Bär F, Mochtar B, Snoep G, Bekkers SC. Eur Heart J. 2006 Apr; 27 (7): 807

25. Abdominal aortic aneurysm screening during transthoracic echocardiography in an unselected population. Bekkers SC, Habets JH, Cheriex EC, Palmans A, Pinto Y, Hofstra L, Crijns HJ. J Am Soc Echocardiogr. 2005 May; 18 (5): 389-93.

26. Tropical endomyocardial fibrosis (Davies' disease): case report demonstrating the role of magnetic resonance imaging. Smedema JP, Winckels SK, Snoep G, Vainer J, Bekkers SC, Crijns HJ. Int J Cardiovasc Imaging. 2004 Dec; 20 (6): 517-22.

27. Diagnostic Value of PET in Cardiac Sarcoidosis. Smedema JP, van Kroonenburgh MJ, Snoep G, Bekkers SC, Gorgels AP. J Nucl Med. 2004 Nov; 45 (11): 1975.

28. Two patients with acute hepatitis B from the same piercing salon. Bekkers SC, Peters FP. Ned Tijdschr Geneeskd. 1999 Dec 11; 143 (50): 2548.

29. Images in cardiology: Intramyocardial dissection after subacute anterior wall myocardial infarction. Bekkers SC, Prenger K, Waltenberger J. Heart. 2005 Sep; 91 (9): e54.

30. End-diastolic myofiber stress and ejection strain increase with ventricular volume overload - serial in-vivo analyses in dogs with complete atrioventricular block. Donker DW, Volders PG, Arts T, Bekkers SC, Hofstra L, Spätjens RL, Beekman JD, Borgers M, Crijns HJ, Vos MA. Basic Res Cardiol. 2005 Jul; 100 (4): 372-82.

31. Massive cardiac involvement in acute lymphatic leukemia. Bekkers SC, Denarié BJ, Bos GM. Heart. 2004 Mar; 90 (3): 354.

32. Widespread regional myocardial transfection by plasmid encoding Del-1 following retrograde coronary venous delivery. Hou D, Maclaughlin F, Thiesse M, Panchal VR, Bekkers SC, Wilson EA, Rogers PI, Coleman MC, March KL. Catheter Cardiovasc Interv. 2003 Feb; 58 (2): 207-11.

33. Nebivolol: a third-generation beta-blocker that augments vascular nitric oxide release: endothelial beta(2)-adrenergic receptor-mediated nitric oxide production. Broeders MA, Doevendans PA, Bekkers SC, Bronsaer R, van Gorsel E, Heemskerk JW, Egbrink MG, van Breda E, Reneman RS, van Der Zee R. Circulation. 2000 Aug 8; 102 (6): 677-84. 
